\title{
Adaptive Control and Robustness in the Gap Metric
}

\author{
M. French \\ School of Electronics and Computer Science, \\ University of Southampton, \\ SO17 1BJ, UK \\ mcf@ecs.soton.ac.uk \\ www.ecs.soton.ac.uk/ ${ }^{\sim m c f}$ \\ Submitted to IEEE Transactions on Automatic Control. \\ Initial submission June 2002
}

This version: April 2005

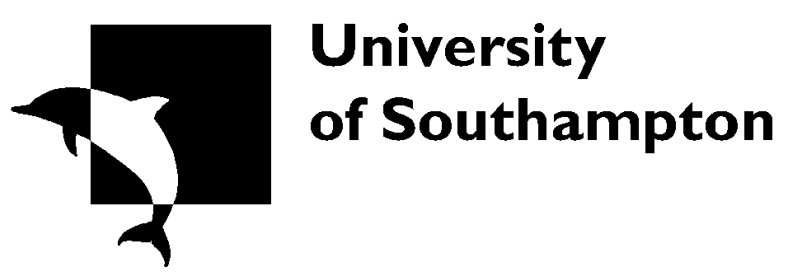

\begin{abstract}
We consider the construction of adaptive controllers for minimum phase linear systems which achieve non-zero robustness margins in the sense of the gap metric. The gap perturbation margin may be more constrained for larger disturbances and for larger parametric uncertainties. Working in an $L^{2}$ setting, and within the framework of the nonlinear gap metric, universal adaptive controllers are first given achieving stabilisation for first order nominal plants, and the results are then generalised to relative degree one nominal plants. Necessary asymptotic properties of the robustness margins are derived for the class of controllers considered. Extensions to the $L^{\infty}$ setting are also developed where two alternative designs are given. A notion of a semi-universal control design is introduced, which is the property that a bound on performance exists which is independent of the a-priori known uncertainty level, and a characterisation is given for when semi-universal designs outperform the class of memoryless controllers and the class of LTI controllers. Robust semi-universal adaptive control designs are given for nominal plants under the classical assumptions of adaptive control in both the $L^{2}$ and $L^{\infty}$ settings. The results are applied throughout to explicit classes of unmodelled dynamics including the Rohrs example.
\end{abstract}




\section{Introduction}

The study of the robustness of adaptive controllers has a long and perhaps infamous history. In the early eighties it was observed that the adaptive designs of the time had limited robustness properties. Closed loops could become unstable even in the presence of small disturbances and innocuous looking classes of unmodelled dynamics. Specifically Rohrs [17] showed that many of the existing designs became unstable even when applied to a first order plant with a pair of unmodelled conjugate poles far out in the left hand plane. These observations gave a great impetus in the 1980's to the study of robust adaptive control [13], where modifications such as dead-zones, $\sigma / e_{1}$ modifications or projection operators (see eg. [16], [19]) are applied to standard adaptive laws to ensure stability in the face of disturbances and classes of unmodelled dynamics. Other strategies include assuming signal richness conditions on reference signals to ensure correct parametric convergence with associated robustness benefits. Extra 'probing-signals' can also be injected into the system to ensure similar effects. The extensive literature on these ideas is summarized in [13]. Typical results (see eg. [11] for a recent example), show robustness of adaptive designs to small uncertainties of a multiplicative type. Singular perturbation analysis can be applied to obtain robustness results for classes of fast unmodelled dynamics (see eg. [13]) (in these results, the stability region shrinks as the speed ratio of the slow (modelled) dynamics to the fast (unmodelled) dynamics decreases). More recently, ideas from inverse optimality [15] have been utilized to achieve robustness to classes of unmodelled input-dynamics, generalising the gain and phase margins of classical control. Inverse optimal results guarantee a disk margin which yields bounds on admissible relative degree zero perturbations [20].

Despite the extensive literature on robust adaptive control, there has remained a wide gulf between the uncertainties considered within adaptive control theory, and the fundamental uncertainty descriptions considered in the theory of linear robust control [26]. As a cornerstone of modern robust control, we have the idea of uncertainties described by (normalized) co-prime factor perturbations, which permits consideration both multiplicative and inverse multiplicative uncertainties within a unified framework. It is well established that the graph topology is the correct framework to study qualitative aspects of robustness to such uncertainties, and further that the gap metric (first introduced into control theory by Zames and El-Sakkary [24], [2]) and its variant the $\nu$ gap [22], induce the graph topology [21], and provide quantitative measures of the uncertainty. Indeed the problem of minimising the robustness margin $b_{P, C}$ lies at the heart of $H^{\infty}$ loop shaping control synthesis [10]. One of the contributions of this paper is therefore to provide adaptive control results within the context of gap metric uncertainties, ie. to provide adaptive results within the framework of the fundamental uncertainty descriptions as developed in robust control theory.

The results in this paper build on recent system-theoretic developments in the study of the robustness of nonlinear feedback systems. Of particular importance for the development of the results in this paper are:

1. The appreciation of the importance of nonlinear gain functions and the corresponding development of nonlinear small gain theorems [14], and,

2. The generalisation of the gap metric to the nonlinear context in [8] (see also [1],[6]), including the development of conditions (of a small gain-type) for robust stability.

The key fundamental paper [8] provides a new framework in which to address the problem of robust adaptive control. A great advantage of the robustness framework of [8] is that the existence of (nonlinear) non-zero robustness margins can be reduced to proving the existence of a certain closed loop gain function. 
In [8] two standard parametric adaptive controllers were considered, and both were shown to have zero-robustness margins in the sense of the margins defined in that paper. Supporting numerical evidence and series expansions of the closed loop solutions suggested that these designs indeed have no robustness to simple but arbitrarily small gap perturbations.

The fundamental question we address is whether it is possible to construct adaptive controllers with non-zero robustness margins. By answering this question in the affirmative, we develop a class of robust adaptive controllers which are robust to both perturbations of the plant in the gap metric and to bounded disturbances. We show (perhaps contrary to expectation), that it is possible to construct a universal adaptive controller for a first order plant which can be arbitrarily unstable, whilst maintaining robustness in a gap metric sense. The gap perturbations may be more constrained for larger disturbances and for larger parametric uncertainty. We give a formulation and solutions to this problem in the case where the gap is measured in either an $L^{2}$ or an $L^{\infty}$ sense.

The constructions require four steps. Firstly we augment the input to the plant so that the uncertain parameters are considered to be fixed constant inputs, (hence the plant is then nonlinear). Secondly we construct a nonlinear adaptive controller for which a gain function relating the external disturbances to the (nonlinear) plant input and output exists. The problem of bounding such gain functions for adaptive controllers is closely related to the problem of bounding non-singular cost functionals, see for example [5]. Thirdly we relate the associated nonlinear robustness margin to linear gap margins on the original linear plant. Finally by a process of input and output injection, non-zero initial conditions are incorporated into the analysis.

Similar results are obtained for minimum phase plants of relative degree one. Interestingly, the controllers obtained are essentially standard parametric adaptive controllers, but with a change in a growth rate in the adaptive law. We illustrate applications of the results to specific classes of unmodelled dynamics: eg. first order lags, an all-pass factor perturbations, the generalised Rohr's dynamics and a general class of co-prime factor uncertainties.

The final contribution of the paper is to extend these constructions to minimum phase plants of arbitrary relative degree. The control design objective is weakened from the construction of a universal controller (ie. one which stabilises the plant irrespective of parameter uncertainty level) to that of a semi-universal control design. A semi-universal design is a control design which can depend on an a-priori bound on the parameter uncertainty level, but whose performance is bounded independent of it. Such controllers are non-conservative in the sense that if the knowledge of the uncertainty level becomes poor, then the performance does not degrade. On the other hand, a conservative control design is defined as a control design whose performance degrades as the knowledge of the uncertainty level becomes poorer. To motivate the significance of the construction of semi-universal designs, we show that memoryless nonlinear controllers are necessarily conservative, and we show that all linear control designs are necessarily conservative. By an intrinsic application of the gap-metric robustness theory, a semi-universal controller is constructed for plants of arbitrary relative degree. This controller achieves non-zero gap robustness margins to unmodelled dynamics and robustness to bounded disturbances.

The results given in this paper therefore construct adaptive controllers with non-zero gap robustness margins. Since the gap metric induces the graph topology which is the fundamental description in which to investigate robustness of closed loops, the results in this paper represent the start of a seemingly natural approach to robust adaptive control.

\section{Background}

The background, notation and nomenclature is largely based on [8]. 
Let $\mathcal{X}$ be a non-empty set and let $0<\omega \leq \infty$. Let $\mathcal{S}_{\omega}$ denote the space of all measurable maps $[0, \omega) \rightarrow \mathcal{X}$, and define $\mathcal{S}=\mathcal{S}_{\infty}$. For $\omega \in(0, \infty], v \in \mathcal{S}_{\omega}$ and $\tau \in[0, \omega), T_{\tau} v \in \mathcal{S}$ is given by

$$
T_{\tau}(v)= \begin{cases}v(t), & t \in[0, \tau) \\ 0, & t \in[\tau, \infty) .\end{cases}
$$

For $\mathcal{V} \subset \mathcal{S}$ and $\omega \in(0, \infty]$, define

$$
\mathcal{V}_{\omega}=\left\{v \in \mathcal{S}_{\omega} \mid T_{\tau} v \in \mathcal{V} \forall \tau \in[0, \omega)\right\}
$$

(with $\mathcal{V}_{\infty} \equiv \mathcal{V}$ ). To $\mathcal{V} \subset \mathcal{S}$ we also associate two larger spaces, with are called the extended space and the ambient space respectively:

$$
\begin{aligned}
& \mathcal{V}_{e}=\left\{v \in \mathcal{S} \mid T_{\tau} v \in \mathcal{V} \forall \tau>0\right\} \\
& \mathcal{V}_{a}=\cup_{\omega \in(0, \infty]} \mathcal{V}_{\omega} .
\end{aligned}
$$

If $v, w \in \mathcal{V}_{a}$, we write $v=w$ if $\left.v\right|_{I}=\left.w\right|_{I}$ where $I=\operatorname{dom}(v) \cap \operatorname{dom}(w)$. Note that $\mathcal{V} \subset \mathcal{V}_{e} \subset \mathcal{V}_{a}$ are strict inclusions. In our applications, frequently $\mathcal{V}$ will be a normed vector space, e.g. $\mathcal{V}=L^{2}[0, \infty)$, in this case, $\mathcal{V}_{e}=L_{\text {loc }}^{2}[0, \infty), \mathcal{V}_{\omega}=L_{\text {loc }}^{2}[0, \omega)$ and $\mathcal{V}_{a}=\cup_{0<\omega \leq \infty} L_{\text {loc }}^{2}[0, \omega)$. It is important to note that $\mathcal{V}_{\omega} \neq L^{2}[0, \omega)$. We will often write $\|x\|_{\tau}=\left\|T_{\tau} x\right\|$.

A mapping $Q: \mathcal{X}_{1} \rightarrow \mathcal{X}_{2}$ is said to be causal if and only if $\tau>0, x, y \in \mathcal{X}_{1}$ s.t. $T_{\tau} x=T_{\tau} y$ implies $T_{\tau} Q x=T_{\tau} Q y$. We consider causal mappings of signal spaces $P: \mathcal{U}_{a} \rightarrow \mathcal{Y}_{a}$ and $C: \mathcal{Y}_{a} \rightarrow \mathcal{U}_{a}$, where $P$ and $C$ represent a plant and a controller, respectively, and $\mathcal{U}$ and $\mathcal{Y}$ are signal spaces over the positive time axis $[0, \infty)$ satisfying the properties: i) $\mathcal{U}, \mathcal{Y}$ are normed vector spaces, ii) $T_{\tau} u \in \mathcal{U}$ for all $u \in \mathcal{U}$, $\tau \geq 0$ (resp. $y \in \mathcal{Y}$ ), and iii) $\left\|T_{\tau} u\right\|<\infty$ for all $\tau \geq 0$ implies $u \in \mathcal{U}$ (resp. $y \in \mathcal{Y}$ ). The signal spaces $L^{p}\left(\mathbb{R}_{+}, \mathbb{R}^{m}\right), 1 \leq p \leq \infty$ satisfy the above assumptions. Let $\mathcal{W}:=\mathcal{U} \times \mathcal{Y}\left(\right.$ with $\mathcal{W}_{e}=\mathcal{U}_{e} \times \mathcal{Y}_{e}$, $\left.\mathcal{W}_{a}=\mathcal{U}_{a} \times \mathcal{Y}_{a}\right)$. Our central concern is with the system of equations:

$$
[P, C]: \quad y_{1}=P u_{1}, \quad u_{2}=C y_{2}, \quad u_{0}=u_{1}+u_{2}, \quad y_{0}=y_{1}+y_{2},
$$

corresponding to the closed-loop feedback configuration of a plant and controller as depicted in Figure 1.

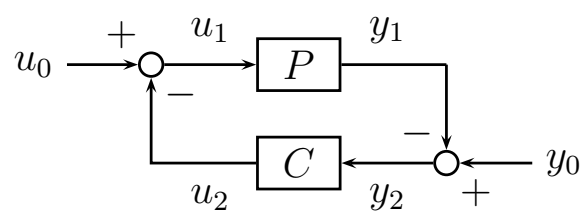

Figure 1: The closed-loop.

Let $w_{i}=\left(u_{i}, y_{i}\right)^{T} \in \mathcal{W}_{a}$ for $i=0,1,2$. Then the closed-loop system $[P, C]$, given by (1), is said to be locally well posed if, and only if,

$$
H_{P, C}: \mathcal{W} \rightarrow \mathcal{W}_{a} \times \mathcal{W}_{a}, w_{0} \mapsto\left(w_{1}, w_{2}\right)
$$

is a causal (single valued) operator. If, in addition, $\operatorname{im} H_{P, C} \subset \mathcal{W}_{e} \times \mathcal{W}_{e}$ then $[P, C]$ is said to be globally well posed, and if im $H_{P, C} \subset \mathcal{W} \times \mathcal{W}$ then $[P, C]$ is said to be BIBO stable. 
If $w_{0} \in \mathcal{W}$, then $\left(w_{1}, w_{2}\right)=H_{P, C}\left(w_{0}\right) \in \mathcal{W}_{a} \times \mathcal{W}_{a}$ is said to have a maximal interval of existence $\left[0, \omega_{w_{0}}\right)$ where $\omega_{w_{0}}=\sup \left\{s>0:\left.\left(w_{1}, w_{2}\right)\right|_{[0, s)} \in \mathcal{W}_{s} \times \mathcal{W}_{s}\right\}$. A globally well posed system is therefore one for which $\omega_{w_{0}}=\infty$ for all $w_{0} \in \mathcal{W}$.

$[P, C]$ is said to be regularly well posed if, and only if, $[P, C]$ is locally well posed and for any $w_{0} \in \mathcal{W}$

$$
T_{\omega_{w_{0}}} H_{P, C}\left(w_{0}\right) \in \mathcal{W} \times \mathcal{W} \Rightarrow \omega_{w_{0}}=\infty
$$

Consequently, if $[P, C]$ is regularly well posed and $T_{\omega_{w_{0}}} H_{P, C}\left(w_{0}\right) \in \mathcal{W} \times \mathcal{W}$ for all $w_{0} \in \mathcal{W}$, then $\omega_{w_{0}}=\infty$ for all $w_{0} \in \mathcal{W}$ and $[P, C]$ is globally well posed.

We remark that regular well posedness usually follows from standard existence and uniqueness properties for differential equations when $\mathcal{W}=L^{\infty}[0, \infty)$. However, when $\mathcal{W} \neq L^{\infty}[0, \infty)$ (as in this paper, where we are also interested in $\mathcal{W}=L^{2}[0, \infty)$ ), stronger properties of the underlying differential equations are required. As shall be shown, all closed-loop systems considered in this paper are regularly well posed.

A causal operator $F: \mathcal{X} \rightarrow \mathcal{V}_{a}$ where $\mathcal{X}, \mathcal{V}$ are subsets of normed signal spaces, is said to be gainfunction stable (or gf-stable) if, and only if, $\operatorname{im} F \subset \mathcal{V}$ and there exists a nonlinear gain function

$$
g[F]:\left(r_{0}, \infty\right) \rightarrow \mathbb{R}_{+}, \quad r \mapsto g[F](r)=\sup _{r_{0}<\left\|T_{\tau} x\right\| \leq r, \tau>0}\left\|T_{\tau} F x\right\|, \quad r_{0}=\inf _{x \in \mathcal{X}}\|x\| .
$$

Often $g[F]$ is class $\mathcal{K}$ (ie. a continuous increasing function $\epsilon:[0, \infty) \rightarrow[0, \infty)$ for which $\epsilon(0)=0)$ or class $\mathcal{K}_{\infty}$ (ie. a class $\mathcal{K}$ function $\epsilon$ with the additional property that $\epsilon(r) \rightarrow \infty$ as $r \rightarrow \infty$ ). Observe further that $\|F x\|_{\tau} \leq g[F]\left(\|x\|_{\tau}\right)$.

A closed-loop system $[P, C]$ is said to be gf-stable if, and only if, it is globally well posed and $H_{P, C}: \mathcal{W} \rightarrow \mathcal{W}_{e} \times \mathcal{W}_{e}$ is gf-stable. Finally, we associate two (projection) operators to a closed-loop system $[P, C]$ :

$$
\Pi_{P / / C}: \mathcal{W} \rightarrow \mathcal{W}_{a}, \quad w_{0} \mapsto w_{1}, \quad \Pi_{C / / P}: \mathcal{W} \rightarrow \mathcal{W}_{a}, \quad w_{0} \mapsto w_{2}
$$

Clearly, $H_{P, C}=\left(\Pi_{P / / C}, \Pi_{C / / P}\right)$ and $\Pi_{P / / C}+\Pi_{C / / P}=I$. Therefore, gf-stability of one of the operators $\Pi_{P / / C}$ and $\Pi_{C / / P}$ implies the gf-stability of the other and so gf-stability of either operator implies gf-stability of the closed-loop system $[P, C]$.

We will be interested in the control of linear SISO minimal $n$-dimensional state space realisations of strictly proper transfer functions in $\mathcal{R}$, i.e. systems of the form

$$
\left.\begin{array}{rl}
\dot{x}(t) & =A x(t)+B u_{1}(t), \quad x(0)=x^{0} \\
y_{1}(t) & =C x(t),
\end{array}\right\}
$$

such that $(A, B, C) \in \mathcal{L}_{n} \subset E_{n}=\mathbb{R}^{n^{2}+2 n}, n \geq 1$, and where

$$
\mathcal{L}_{n}=\left\{(A, B, C) \in E_{n} \mid(A, B, C) \text { is stabilizable and detectable }\right\} .
$$

For normed signal spaces $\mathcal{U}, \mathcal{Y}$ and $\left(\theta, x^{0}\right) \in \mathcal{L}_{n} \times \mathbb{R}^{n}$, we define the plant operator

$$
P^{*}\left(\theta, x^{0}\right): \mathcal{U}_{a} \rightarrow \mathcal{Y}_{a}, \quad u_{1} \mapsto P^{*}\left(\theta, x^{0}\right)\left(u_{1}\right):=y_{1}
$$

where $u_{1} \in \mathcal{U}_{a}, y_{1} \in \mathcal{Y}_{a}$ satisfy (6) for the initial value $x(0)=x^{0}$ and $\theta=(A, B, C)$. Note that $P^{*}$ is a map from $\mathcal{L}=\bigcup_{n \geq 1}\left(\mathcal{L}_{n} \times \mathbb{R}^{n}\right)$ to the space of maps $\mathcal{U}_{a} \rightarrow \mathcal{Y}_{a}$. Furthermore, the operator $P^{*}\left(\theta, x^{0}\right)$ corresponds to a realisation of the transfer function $C\left(s I_{n}-A\right)^{-1} B \in \mathcal{R}$. We will also utilize the formal transfer function symbol $G(s)=C\left(s I_{n}-A\right)^{-1} B$ to represent the linear time-domain operator $P^{*}((A, B, C), 0)$. 
Corresponding to a plant operator $P$ is a subset of $\mathcal{W}$, called the graph of the plant $\mathcal{G}_{P}$, which is defined as

$$
\mathcal{G}_{P}=\left\{\left(\begin{array}{c}
u \\
P u
\end{array}\right) \mid u \in \mathcal{U}, P u \in \mathcal{Y}\right\} \subset \mathcal{W} .
$$

Similarly the graph of a controller operator $C$ is defined as

$$
\mathcal{G}_{C}=\left\{\left(\begin{array}{c}
C y \\
y
\end{array}\right) \mid C y \in \mathcal{U}, y \in \mathcal{Y}\right\} \subset \mathcal{W} .
$$

It is often useful to use an image representation of the graph of an operator. For linear systems, where $\mathcal{W}=L^{p}[0, \infty) \times L^{p}[0, \infty), p=2, \infty$, such a representation [21] is of the form:

$$
\mathcal{G}_{P}=\left(\begin{array}{c}
A(s) \\
B(s)
\end{array}\right) L^{p}[0, \infty)
$$

where $A(s), B(s), V(s), U(s) \in \mathcal{R H}^{\infty}$ satisfy the Bezout identity: $V(s) A(s)+U(s) B(s)=1$ and $P(s)=$ $B(s) / A(s)$, where observe we are expressing a time-domain linear operator by its frequency domain symbol: these graphs should be interpreted as time domain subsets of the signal space (we will do this throughout the paper).

Let $\Gamma$ denote the set of all causal operators $\mathcal{U}_{a} \rightarrow \mathcal{Y}_{a}$. Given $P_{1}, P_{2} \in \Gamma$ and a normed signal space $\mathcal{V}$, define the (possibly empty) set

$$
\mathcal{O}_{P_{1}, P_{2}}=\left\{\Phi: \mathcal{G}_{P_{1}} \rightarrow \mathcal{G}_{P_{2}} \mid \Phi \text { is causal, bijective, and } \Phi(0)=0\right\}
$$

and the nonlinear directed gap:

$$
\vec{\delta}_{\mathcal{V}}\left(P_{1}, P_{2}\right)= \begin{cases}\inf _{\Phi \in \mathcal{O}_{P_{1}, P_{2}}} \sup _{x \in \mathcal{G}_{P_{1}} \backslash\{0\}, \tau>0} \frac{\left\|\left.T_{\tau}(\Phi-I)\right|_{\mathcal{G}_{1}} x\right\|_{\mathcal{V}}}{\left\|T_{\tau} x\right\|_{\mathcal{V}}} & \text { if } \mathcal{O}_{P_{1}, P_{2}} \neq \emptyset \\ \infty & \text { if } \mathcal{O}_{P_{1}, P_{2}}=\emptyset\end{cases}
$$

The nonlinear gap $\delta\left(P_{1}, P_{2}\right)=\delta_{\mathcal{V}}\left(P_{1}, P_{2}\right)$, [8], is defined to be

$$
\delta_{\mathcal{V}}: \Gamma \times \Gamma \rightarrow[0, \infty], \quad \delta_{\mathcal{V}}\left(P_{1}, P_{2}\right)=\max \left\{\vec{\delta}_{\mathcal{V}}\left(P_{1}, P_{2}\right), \vec{\delta}_{\mathcal{V}}\left(P_{2}, P_{1}\right)\right\}
$$

The $L^{2}[0, \infty)$ nonlinear gap is a generalisation of the standard definition of the $H_{2}$ gap $\delta_{0}(\cdot, \cdot)[24]$, [8], in the sense that if $P_{1}, P_{2} \in \mathcal{R}$, and $\delta_{0}\left(P_{1}, P_{2}\right)<1$, then it is shown in [8, Proposition 5] that

$$
\vec{\delta}_{0}\left(P_{1}, P_{2}\right)=\vec{\delta}_{L^{2}}\left(P_{1}, P_{2}\right)
$$

The topology induced on $\mathcal{R}$ by the gap is called the graph topology [21, p235]; note that the graph topology on $\Gamma$ induces the graph topology on $\mathcal{R}$ via the subset topology and the Laplace transform.

\section{The First Order $L^{2}$ result}

We first develop a robustness result for the case of a first order linear system perturbed by $L^{2}$ disturbances. This result then forms the prototype for the more general results that follow. By presenting the simplest system first, we can motivate the development of the general machinery, whilst keeping the technicalities of the specific closed loop to a minimum. We will generalise the class of plants under consideration in Section 8. 


\subsection{Statement of the result}

The main scalar result is as follows:

Theorem 3.1. Let $\mathcal{U}=\mathcal{Y}=L^{2}[0, \infty)$, and consider $P^{*}\left((a, 1,1), y_{1}^{0}\right): \mathcal{U}_{a} \rightarrow \mathcal{Y}_{a}$ :

$$
P^{*}\left((a, 1,1), y_{1}^{0}\right)\left(u_{1}\right)=y_{1}: \dot{y}_{1}=a y_{1}+u_{1}, y_{1}(0)=y_{1}^{0} \in \mathbb{R}, a \in \mathbb{R}
$$

Then there exists a controller $C_{L^{2}}^{*}: \mathcal{Y}_{a} \rightarrow \mathcal{U}_{a}$ and a function $\mu: \mathbb{R}_{+} \times \mathbb{R} \rightarrow(0, \infty)$ such that if $\theta_{1} \in \mathcal{L}$ satisfies

$$
\vec{\delta}\left(P^{*}((a, 1,1), 0), P^{*}\left(\theta_{1}, 0\right)\right) \leq \mu\left(r_{0}, a\right),
$$

for some $a \in \mathbb{R}$ and $r_{0}>0$ then there exists $\lambda>0$ such that

$$
\lambda\left|x_{1}^{0}\right|+\left\|\left(\begin{array}{c}
u_{0} \\
y_{0}
\end{array}\right)\right\| \leq r_{0} \Rightarrow H_{P_{1}^{*}\left(\theta_{1}, x_{1}^{0}\right), C_{L^{2}}^{*}}\left(\begin{array}{c}
u_{0} \\
y_{0}
\end{array}\right) \in L^{2}[0, \infty) \times L^{2}[0, \infty) .
$$

Notice that the margin $\mu\left(r_{0}, a\right)$ is strictly positive. Furthermore a suitable controller $C_{L^{2}}^{*}: \mathcal{Y}_{a} \rightarrow \mathcal{U}_{a}$ can be explicitly constructed as follows:

$$
\begin{aligned}
C_{L^{2}}^{*}\left(y_{2}\right):=u_{2} & =-\hat{k} y_{2}-y_{2} \\
\hat{k}(t) & =\left\|\sqrt{\alpha} y_{2}\right\|_{L^{2}[0, t]}^{\frac{1}{2}}=\left(\int_{0}^{t} \alpha y_{2}^{2} d t\right)^{\frac{1}{4}} .
\end{aligned}
$$

Here, and throughout, the adaptive gain $\alpha$ is strictly positive. Note that the above adaptive law (equation (15)) is similar to the standard parametric adaptive law: $\dot{\hat{k}}=\alpha y_{2}^{2}, \hat{k}(0)=0$ which can be equivalently expressed as: $\hat{k}(t)=\left\|\sqrt{\alpha} y_{2}\right\|_{L^{2}[0, t]}^{2}=\int_{0}^{t} \alpha y_{2}^{2} d t$. It is also worthwhile to observe that the adaptive law (15) can be written in the equivalent differential form:

$$
\dot{\hat{k}}=\alpha \frac{1}{4 \hat{k}^{3}} y_{2}^{2}, \quad \hat{k}(0)=0
$$

where the singularity at $\hat{k}=0$ (eg. when $t=0)$ is non-essential.

\subsection{Applications}

Before proceeding to the proof of the result, we discuss how the theorem may be applied. It may appear restrictive that the size of the gap perturbation is constrained by the size of the disturbance signal this appears to be an essential feature due to the nature of the adaptive control. However, typical applications of Theorem 3.1 are to uncertainty sets which are asymptotically null in the following sense:

Definition 3.2. A plant uncertainty set $\Delta\left(P^{*}(\theta, 0), \epsilon\right) \subset \mathcal{R}$ parameterised by a nominal plant $P^{*}(\theta, 0)$, $\theta \in \mathcal{L}_{n}$ and an uncertainty level $\epsilon>0$, with the property that

$$
\lim _{\epsilon \rightarrow 0} \sup _{\widetilde{P} \in \Delta\left(P^{*}(\theta, 0), \epsilon\right)} \vec{\delta}\left(P^{*}(\theta, 0), \widetilde{P}\right)=0,
$$

is said to be asymptotically null.

All the following applications of the main theorem are then achieved using the corollary: 
Corollary 3.2.1. Let $\mathcal{U}=\mathcal{Y}=L^{2}[0, \infty)$, and let $P^{*}\left((a, 1,1), y_{1}^{0}\right): \mathcal{U}_{a} \rightarrow \mathcal{Y}_{a}$ be defined:

$$
y_{1}=P^{*}\left((a, 1,1), y_{1}^{0}\right)\left(u_{1}\right): \dot{y}_{1}=a y_{1}+u_{1}, y_{1}(0)=y_{1}^{0} \in \mathbb{R}, a \in \mathbb{R} .
$$

Suppose $\Delta\left(P^{*}((a, 1,1), 0), \cdot\right)$ is asymptotically null. Then there exists a controller $C_{L^{2}}^{*}: \mathcal{Y}_{a} \rightarrow \mathcal{U}_{a}$ for which for all $r>0$, there exists $\epsilon>0$ such that if $\widetilde{P} \in \Delta\left(P^{*}((a, 1,1), 0), \epsilon\right)$ and $\left\|\left(u_{0}, y_{0}\right)^{T}\right\| \leq r$ then $H_{\widetilde{P}, C_{L^{2}}^{*}}\left(u_{0}, y_{0}\right) \in L^{2}[0, \infty) \times L^{2}[0, \infty)$.

Proof. This follows straightforwardly from Theorem 3.1 and Definition 3.2.

\subsubsection{The generalised Rohrs example}

Firstly we consider the Rohrs example [17]. This example has historical importance since it first drew the attention of adaptive control researchers to the instabilities of adaptive controllers in the presence of un-modelled dynamics. ${ }^{1}$ The Rohrs example consists of the nominal first order plant

$$
\frac{2}{s+1}
$$

perturbed multiplicatively by a pair of highly damped conjugate poles. The multiplicative perturbation considered was:

$$
\frac{229}{s^{2}+30 s+229}
$$

corresponding to poles at $-15 \pm 2 i$. We define the generalised Rohrs perturbation as follows:

$$
\Delta(P, \epsilon)=\left\{P_{1}: \mathcal{U} \rightarrow \mathcal{Y}: \widetilde{P}=P \frac{|\lambda|^{2}}{(s-\lambda)\left(s-\lambda^{*}\right)}, \operatorname{Re} \lambda \leq-\epsilon^{-1}\right\}
$$

We claim that this uncertainty set is asymptotically null w.r.t. to the plant $P^{*}((a, 1,1), 0){ }^{2}$

To prove this asssertion, consider $0<e<1, e \neq-a$ and observe that the graphs of $P^{*}((a, 1,1), 0)$, $\widetilde{P}$ can be written as:

$$
\begin{aligned}
\mathcal{G}_{P^{*}((a, 1,1), 0)} & =\left(\begin{array}{c}
A \\
B
\end{array}\right) L^{2}[0, \infty)=\left(\begin{array}{c}
\frac{s-a}{s+e} \\
\frac{1}{s+e}
\end{array}\right) L^{2}[0, \infty) \\
\mathcal{G}_{\widetilde{P}} & =\left(\begin{array}{c}
A^{\prime} \\
B^{\prime}
\end{array}\right) L^{2}[0, \infty)=\left(\begin{array}{c}
\frac{s-a}{s+1} \\
\frac{|\lambda|^{2}}{(s+1)(s-\lambda)\left(s-\lambda^{*}\right)}
\end{array}\right) L^{2}[0, \infty) .
\end{aligned}
$$

Let $(V, U):=(1, e+a)$ (which satisfies $\left.(V, U)\left(\begin{array}{c}A \\ B\end{array}\right)=1\right)$, and define the mapping $\Phi: \mathcal{G}_{P^{*}((a, 1,1), 0)} \rightarrow \mathcal{G}_{\widetilde{P}}$ :

$$
\Phi:=\left(\begin{array}{c}
A^{\prime} \\
B^{\prime}
\end{array}\right)(V, U)
$$

\footnotetext{
${ }^{1}$ Note that all the instabilities exhibited by Rohrs (and the closed loop operator infinite gain explanation given) are demonstrated for tracking problems, or to set point regulation problems with constant disturbances. Rohrs did not investigate $L^{2}$ disturbances for stabilisation, which is the setting in which we are working initially.

${ }^{2}$ The Rohr's plant is $P^{*}((1,2,1), 0)$, but by re-scaling of the signals, the conclusions for $P^{*}((a, 1,1), 0)$ are also valid for plants of the form $P^{*}((a, b, c), 0)$.
} 
Then,

$$
\begin{aligned}
\|\left.(I-\Phi)\right|_{\mathcal{G}_{P^{*}((a, 1,1), 0)} \|_{L^{2}}} & =\left\|\left(\begin{array}{c}
A \\
B
\end{array}\right)(V, U)-\left(\begin{array}{c}
A^{\prime} \\
B^{\prime}
\end{array}\right)(V, U)\right\|_{H^{\infty}} \\
& =\left\|\left(\begin{array}{c}
0 \\
\frac{s^{2}-2 \operatorname{Re} \lambda s}{(s+1)(s-\lambda)\left(s-\lambda^{*}\right)}
\end{array}\right)(1, e+a)\right\|_{H^{\infty}} \\
& \leq \sqrt{\left(a^{2}+2 e a+1+e^{2}\right)}\left\|\frac{s}{s+1}\right\|\left\|_{H^{\infty}}\right\| \frac{s-2 \operatorname{Re} \lambda}{(s-\lambda)\left(s-\lambda^{*}\right)} \|_{H^{\infty}} \\
& \leq \sqrt{\left(a^{2}+2 e a+1+e^{2}\right)} \sup _{\omega \in \mathbb{R}} \sqrt{\frac{1}{(\operatorname{Re} \lambda)^{2}}\left(\frac{\omega^{2}+4(\operatorname{Re} \lambda)^{2}}{\omega^{2}+(\operatorname{Re} \lambda)^{2}}\right)} \\
& \leq 2 \sqrt{\epsilon\left(a^{2}+2 e a+1+e^{2}\right)} \\
& \rightarrow 0 \text { as } \epsilon \rightarrow 0,
\end{aligned}
$$

as required. Consequently, Corollary 3.2.1 establishes that for sufficiently small $\epsilon>0$ (which depends on the disturbance level $r$ ) the controller $C_{L^{2}}^{*}$ also stabilizes all plants in the set $\Delta\left(P^{*}((a, 1,1), 0), \epsilon\right)$.

\subsubsection{Other perturbations}

By similar computations, we can exhibit many interesting uncertainty sets which are asymptotically null. For example, consider a nominal plant perturbed by a series cascade of a first order lag and an all-pass factor:

$$
\Delta\left(P^{*}((a, 1,1), 0), \epsilon\right)=\left\{\widetilde{P}: \mathcal{U}_{a} \rightarrow \mathcal{Y}_{a}: \widetilde{P}=\frac{M(N-s)}{(s+M)(s+N)(s+a)}, \quad M, N \geq \epsilon^{-1} \geq 1\right\}
$$

This uncertainty set is asymptotically null w.r.t. to the plant $P^{*}((a, 1,1), 0)$, ie. the perturbation is vanishingly small in a gap sense as $N, M$ become large. Corollary 3.2.1 establishes that for sufficiently large $M, N$ (which depends on the disturbance level $r$ ) the controller $C_{L^{2}}^{*}$ also stabilizes the perturbed plant:

$$
\frac{M(N-s)}{(s-a)(s+M)(s+N)} \text {. }
$$

From the perspective of classical adaptive control, this example is of interest since it violates all the classical assumptions; namely that the relative degree, sign of the high frequency gain and system order differ from the nominal plant. Furthermore, the perturbed plants are not minimum phase.

Finally we observe that the gap metric precisely measures the size of the smallest co-prime factor perturbations, hence, if $\widetilde{P}_{1}(s)=B(s) A(s)^{-1}$ has normalized co-prime factors $A$, $B$, then the following set is asymptotically null:

$$
\Delta\left(\widetilde{P}_{1}, \epsilon\right)=\left\{\widetilde{P}_{2}: \mathcal{U}_{a} \rightarrow \mathcal{Y}_{a}: \Delta_{A}, \Delta_{B} \in H^{\infty}, \widetilde{P}_{2}=\frac{B+\Delta_{B}}{A+\Delta_{A}}, \quad\left\|\left(\begin{array}{c}
\Delta_{A} \\
\Delta_{B}
\end{array}\right)\right\|_{H^{\infty}} \leq \epsilon\right\} .
$$

\section{Properties of an $L^{2}$ adaptive controller}

\subsection{Well posedness}

Let $\mathcal{U}=\mathcal{Y}=L^{2}[0, \infty)$, and consider the controller $C_{L^{2}}^{*}$ defined by (15). We first establish regular well-posedness of this controller when connected to any linear plant in $\mathcal{L}$. 
Lemma 4.1. Let $\mathcal{U}=\mathcal{Y}=L^{2}[0, \infty)$ and suppose $\left(\theta, x_{1}^{0}\right) \in \mathcal{L}_{n} \times \mathbb{R}^{n}$ for some $n \geq 1$. Then the feedback interconnection $\left[P^{*}\left(\theta, x_{1}^{0}\right), C_{L^{2}}^{*}\right]$ is regularly well posed.

Proof. $\left[P^{*}\left(\theta, x_{1}^{0}\right), C_{L^{2}}^{*}\right]$ is locally well posed by standard results on ordinary differential equations. Suppose $w_{0} \in \mathcal{W}$, and consider $\left(w_{1}, w_{2}\right)=H_{P^{*}(\theta, 0), C_{L^{2}}^{*}}\left(w_{0}\right)$ where $\operatorname{dom}\left(w_{1}, w_{2}\right)=[0, \omega)$ is maximal. Suppose $T_{\omega}\left(w_{1}, w_{2}\right) \in \mathcal{W} \times \mathcal{W}$. Then $y_{1} \in L^{2}[0, \omega)$ hence since for $0 \leq t<\omega, k(t)=\left\|y_{2}\right\|_{L^{2}[0, t]}^{\frac{1}{2}} \leq$ $\left(\left\|y_{0}\right\|_{L^{2}[0, t]}+\left\|y_{1}\right\|_{L^{2}[0, t]}\right)^{\frac{1}{2}}$, it follows that $k$ is uniformly bounded on $[0, \omega)$. Since the $x$ sub-system of $\left[P^{*}\left(\theta, x_{1}^{0}\right), C_{L^{2}}^{*}\right]$ is a linear time-varying system on $[0, \omega)$ with an $L^{2}$ input, finite escape time is not possible, hence $\omega=\infty$ as required.

Proposition 4.2. Let $\mathcal{U}=\mathcal{Y}=L^{2}[0, \infty)$ and suppose $a, y_{1}^{0} \in \mathbb{R}$. Then the feedback interconnection $\left[P^{*}\left((a, 1,1), y_{1}^{0}\right), C_{L^{2}}^{*}\right]$ is BIBO stable.

Proof. It suffices to demonstrate that $u_{0}, y_{0} \in L^{2}[0, \infty)$ implies $u_{1}, y_{1} \in L^{2}[0, \infty)$, since the corresponding properties for $u_{2}, y_{2}$ follow from the parallel projection properties. So, let $\left(u_{0}, y_{0}\right)^{T} \in \mathcal{W}$, let $[0, \omega)$ be the maximal interval of existence, and let $0 \leq t^{*}<\omega$ be defined as $t^{*}=\inf \{t \geq 0: \hat{k}(t) \geq a\}$ if the infimum exists, and $t^{*}=\omega$ otherwise. Then:

$\left\|y_{1}\right\|_{L^{2}\left[0, t^{*}\right]} \leq\left\|y_{2}\right\|_{L^{2}\left[0, t^{*}\right]}+\left\|y_{0}\right\|_{L^{2}\left[0, t^{*}\right]}=\frac{1}{\sqrt{\alpha}} \hat{k}\left(t^{*}\right)^{2}+\left\|y_{0}\right\|_{L^{2}\left[0, t^{*}\right]} \leq \frac{1}{\sqrt{\alpha}} a^{2}+\left\|y_{0}\right\|_{L^{2}\left[0, t^{*}\right]} \leq \frac{1}{\sqrt{\alpha}} a^{2}+\left\|y_{0}\right\|_{L^{2}[0, \infty)}$.

Suppose $t^{*}<\omega$. We first bound $y_{1}\left(t^{*}\right)$. Define $V: \mathbb{R} \rightarrow \mathbb{R}_{+}$as $V\left(y_{1}\right)=\frac{1}{2} y_{1}^{2}$. Now,

$$
\dot{V}=y_{1} \dot{y}_{1}=(a-\hat{k}-1) y_{1}^{2}+u_{0} y_{1}+y_{0} y_{1}+\hat{k} y_{0} y_{1}
$$

and applying Young's inequality $\left(a b-\frac{1}{4} b^{2} \leq a^{2}\right)$ twice we obtain:

$$
\begin{aligned}
\dot{V} & \leq-\frac{1}{2} y_{1}^{2}+(1+\hat{k})^{2} y_{0}^{2}+u_{0}^{2}+(a-\hat{k}) y_{1}^{2} \\
& \leq-\frac{1}{2} y_{1}^{2}+3\left(1+\hat{k}^{2}\right) y_{0}^{2}+u_{0}^{2}+(|a|+|\hat{k}|) y_{1}^{2}
\end{aligned}
$$

By integrating, and by observing that $\hat{k}$ is non-negative and increasing, we obtain:

$$
\begin{aligned}
V\left(y_{1}\left(t^{*}\right)\right)-V\left(y_{1}(0)\right) & =\int_{0}^{t^{*}} \dot{V} d t \\
& \leq-\frac{1}{2}\left\|y_{1}\right\|_{L^{2}\left[0, t^{*}\right]}^{2}+3\left(1+\hat{k}^{2}\left(t^{*}\right)\right)\left\|y_{0}\right\|_{L^{2}\left[0, t^{*}\right]}^{2}+\left\|u_{0}\right\|_{L^{2}\left[0, t^{*}\right]}^{2}+\left(|a|+\left|\hat{k}\left(t^{*}\right)\right|\right)\left\|y_{1}\right\|_{L^{2}\left[0, t^{*}\right]}^{2} \\
& \leq-\frac{1}{2}\left\|y_{1}\right\|_{L^{2}\left[0, t^{*}\right]}^{2}+3\left(1+a^{2}\right)\left\|y_{0}\right\|_{L^{2}\left[0, t^{*}\right]}^{2}+\left\|u_{0}\right\|_{L^{2}\left[0, t^{*}\right]}^{2}+2|a|\left\|y_{1}\right\|_{L^{2}\left[0, t^{*}\right]}^{2},
\end{aligned}
$$

which implies:

$$
\begin{aligned}
y_{1}^{2}\left(t^{*}\right) & \leq 2 V(0)-\left\|y_{1}\right\|_{L^{2}\left[0, t^{*}\right]}^{2}+6\left(1+|a|^{2}\right)\left\|y_{0}\right\|_{L^{2}\left[0, t^{*}\right]}^{2}+2\left\|u_{0}\right\|_{L^{2}\left[0, t^{*}\right]}^{2}+4|a|\left\|y_{1}\right\|_{L^{2}\left[0, t^{*}\right]}^{2}, \\
& \leq\left(y_{1}^{0}\right)^{2}+6\left(1+|a|^{2}\right)\left\|y_{0}\right\|_{L^{2}\left[0, t^{*}\right]}^{2}+2\left\|u_{0}\right\|_{L^{2}\left[0, t^{*}\right]}^{2}+4|a|\left(\frac{1}{\sqrt{\alpha}} a^{2}+\left\|y_{0}\right\|_{L^{2}\left[0, t^{*}\right]}\right)^{2} \\
& \leq\left(y_{1}^{0}\right)^{2}+6\left(1+|a|^{2}\right)\left\|y_{0}\right\|_{L^{2}[0, \infty)}^{2}+2\left\|u_{0}\right\|_{L^{2}[0, \infty)}^{2}+4|a|\left(\frac{1}{\sqrt{\alpha}} a^{2}+\left\|y_{0}\right\|_{L^{2}[0, \infty)}\right)^{2} .
\end{aligned}
$$


We now consider the $L^{2}$ estimates on $\left[t^{*}, \omega\right)$. Since $\hat{k}$ is increasing it follows that $a-\hat{k} \leq 0$ for $\omega>t \geq t^{*}$, hence we can establish an inequality of the form:

$$
\dot{V} \leq-y_{1}^{2}+u_{0} y_{1}+y_{0} y_{1}+\hat{k} y_{0} y_{1} \leq-\frac{1}{2} y_{1}^{2}+3\left(\left(1+\hat{k}^{2}\right)\right) y_{0}^{2}+u_{0}^{2}
$$

Since $\hat{k}$ is increasing, by integrating on $\left[t^{*}, t\right)$, we obtain:

$$
V\left(y_{1}(t)\right)-V\left(y_{1}\left(t^{*}\right)\right)=\int_{t^{*}}^{t} \dot{V} d t \leq-\frac{1}{2}\left\|y_{1}\right\|_{L^{2}\left[t^{*}, t\right)}^{2}+3\left(1+\hat{k}^{2}(t)\right)\left\|y_{0}\right\|_{L^{2}\left[t^{*}, t\right)}^{2}+\left\|u_{0}\right\|_{L^{2}\left[t^{*}, t\right)}^{2}
$$

which implies that for all $t \geq t^{*}$,

$$
\left\|y_{1}\right\|_{L^{2}\left[t^{*}, t\right)}^{2} \leq y_{1}^{2}\left(t^{*}\right)+6\left(1+\hat{k}^{2}(t)\right)\left\|y_{0}\right\|_{L^{2}\left[t^{*}, t\right)}^{2}+2\left\|u_{0}\right\|_{L^{2}\left[t^{*}, t\right)}^{2}
$$

Now let us estimate $\hat{k}(t)$ for $t \in\left[t^{*}, \omega\right)$. From the definition of the adaptive law (15), we have:

$$
\hat{k}^{4}(t)-\hat{k}^{4}\left(t^{*}\right)=\hat{k}^{4}(t)-a^{4}=\alpha\left\|y_{2}\right\|_{L^{2}\left[t^{*}, t\right)}^{2} \leq \alpha\left(\left\|y_{1}\right\|_{L^{2}\left[t^{*}, t\right)}+\left\|y_{0}\right\|_{L^{2}\left[t^{*}, t\right)}\right)^{2} \leq 3 \alpha\left(\left\|y_{1}\right\|_{L^{2}\left[t^{*}, t\right)}^{2}+\left\|y_{0}\right\|_{L^{2}\left[t^{*}, t\right)}^{2}\right),
$$

and in particular by the inequality $(1+a)^{\frac{1}{2}} \leq 1+\frac{a}{2}$ we obtain:

$$
\begin{aligned}
\hat{k}^{2}(t) & \leq\left(a^{4}+3 \alpha\left(\left\|y_{1}\right\|_{L^{2}\left[t^{*}, t\right)}^{2}+\left\|y_{0}\right\|_{L^{2}\left[t^{*}, t\right)}^{2}\right)\right)^{\frac{1}{2}}, \\
& \leq \sqrt{3 \alpha}\left\|y_{1}\right\|_{L^{2}\left[t^{*}, t\right)}\left(1+\frac{a^{4}+3 \alpha\left\|y_{0}\right\|_{L^{2}\left[t^{*}, t\right)}^{2}}{6 \alpha\left\|y_{1}\right\|_{L^{2}\left[t^{*}, t\right)}^{2}}\right) .
\end{aligned}
$$

Substituting inequality (37) into inequality (35), we obtain:

$$
\left\|y_{1}\right\|_{L^{2}\left[t^{*}, t\right)}^{2} \leq y_{1}^{2}\left(t^{*}\right)+6\left(1+\sqrt{3 \alpha}\left\|y_{1}\right\|_{L^{2}\left[t^{*}, t\right)}\left(1+\frac{a^{4}+3 \alpha\left\|y_{0}\right\|_{L^{2}\left[t^{*}, t\right)}^{2}}{6 \alpha\left\|y_{1}\right\|_{L^{2}\left[t^{*}, t\right)}^{2}}\right)\right)\left\|y_{0}\right\|_{L^{2}\left[t^{*}, t\right)}^{2}+2\left\|u_{0}\right\|_{L^{2}\left[t^{*}, t\right)}^{2},
$$

Rearranging and letting $t \rightarrow \omega:$

$$
\begin{aligned}
\left\|y_{1}\right\|_{L^{2}\left[t^{*}, \omega\right) \leq}^{3} \leq\left(6 \sqrt{3 \alpha}\left\|y_{0}\right\|_{L^{2}\left[t^{*}, \omega\right)}\right)\left\|y_{1}\right\|_{L^{2}\left[t^{*}, \omega\right)}^{2} & +\left(y_{1}^{2}\left(t^{*}\right)+6\left\|y_{0}\right\|_{L^{2}\left[t^{*}, \omega\right)}^{2}+2\left\|u_{0}\right\|_{L^{2}\left[t^{*}, \omega\right)}^{2}\right)\left\|y_{1}\right\|_{L^{2}\left[t^{*}, \omega\right)} \\
& +\sqrt{\frac{3}{\alpha}}\left(a^{4}+3 \alpha\left\|y_{0}\right\|_{L^{2}\left[t^{*}, \omega\right)}^{2}\right)\left\|y_{0}\right\|_{L^{2}\left[t^{*}, \omega\right)}^{2} \\
\leq\left(6 \sqrt{3 \alpha}\left\|y_{0}\right\|_{L^{2}[0, \infty)}\right)\left\|y_{1}\right\|_{L^{2}\left[t^{*}, \omega\right)}^{2} & +\left(y_{1}^{2}\left(t^{*}\right)+6\left\|y_{0}\right\|_{L^{2}[0, \infty)}^{2}+2\left\|u_{0}\right\|_{L^{2}[0, \infty)}^{2}\right)\left\|y_{1}\right\| L_{L^{2}\left[t^{*}, \omega\right)} \\
& +\sqrt{\frac{3}{\alpha}}\left(a^{4}+3 \alpha\left\|y_{0}\right\|_{L^{2}[0, \infty)}^{2}\right)\left\|y_{0}\right\|_{L^{2}[0, \infty)}^{2}
\end{aligned}
$$

Since the r.h.s of inequality (39) is quadratic in $\left\|y_{1}\right\|_{L^{2}\left[t^{*}, \omega\right)}$ with positive coefficients, it follows that $\left\|y_{1}\right\|_{L^{2}\left[t^{*}, \omega\right)}$ is bounded as a function of $\left|y_{1}\left(t^{*}\right)\right|,|a|,\left\|y_{0}\right\|_{L^{2}[0, \infty)},\left\|u_{0}\right\|_{L^{2}[0, \infty)}$. Furthermore, the cubic inequality (39) can be solved explicitly to give this bound (see later). Since we have bounded $\left\|y_{1}\right\|_{L^{2}\left[0, t^{*}\right)}$ in terms of $|a|,\left\|y_{0}\right\|_{L^{2}[0, \infty)}$, and $\left|y_{1}\left(t^{*}\right)\right|$ in terms of $\left|y_{1}^{0}\right|,|a|,\left\|y_{0}\right\|_{L^{2}[0, \infty)},\left\|u_{0}\right\|_{L^{2}[0, \infty)}$ it follows that we have bounded $\left\|y_{1}\right\|_{L^{2}[0, \omega)}$ in terms of:

$$
\left|y_{1}^{0}\right|,|a|,\left\|y_{0}\right\|_{L^{2}[0, \infty)},\left\|u_{0}\right\|_{L^{2}[0, \infty)},
$$


as required. Since the closed loop $\left[P^{*}\left((a, 1,1), y_{1}^{0}\right), C_{L^{2}}^{*}\right]$ is regularly well posed by Lemma 4.1 , it follows that $\omega=\infty$. A similar bound for $\left\|u_{1}\right\|_{L^{2}[0, \infty)}$ can now also be found, since:

$$
\begin{aligned}
& \left\|u_{1}\right\|_{L^{2}[0, \infty)}=\left\|u_{0}-u_{2}\right\|_{L^{2}[0, \infty)} \\
& \leq\left\|u_{0}\right\|_{L^{2}[0, \infty)}+\left\|-\hat{k}\left(y_{0}-y_{1}\right)-\left(y_{0}-y_{1}\right)\right\|_{L^{2}[0, \infty)} \\
& \leq\left\|u_{0}\right\|_{L^{2}[0, \infty)}+\|\hat{k}\|_{L^{\infty}[0, \infty)}\left(\left\|y_{0}\right\|_{L^{2}[0, \infty)}+\left\|y_{1}\right\|_{L^{2}[0, \infty)}\right)+\left\|y_{0}\right\|_{L^{2}[0, \infty)}+\left\|y_{1}\right\|_{L^{2}[0, \infty)} \\
& \leq\left\|u_{0}\right\|_{L^{2}[0, \infty)}+\alpha^{\frac{1}{4}}\left(\left\|y_{0}\right\|_{L^{2}[0, \infty)}+\left\|y_{1}\right\|_{L^{2}[0, \infty)}\right)^{\frac{3}{2}}+\left\|y_{0}\right\|_{L^{2}[0, \infty)}+\left\|y_{1}\right\|_{L^{2}[0, \infty)} .
\end{aligned}
$$

Hence it follows that $\left\|u_{1}\right\|_{L^{2}[0, \infty)}$ is bounded as a function of $\left|y_{1}^{0}\right|,|a|,\left\|y_{0}\right\|_{L^{2}[0, \infty)},\left\|u_{0}\right\|_{L^{2}[0, \infty)}$, hence completing the proof.

\subsection{Incorporation of parameterisations}

The first observation is that for $a>0$, the operator $H_{P^{*}((a, 1,1), 0), C_{L^{2}}^{*}}$ does not have even a local finite gain (or a class $\mathcal{K}$ gain function), since

$$
\left(u_{0}, y_{0}\right) \approx 0 \quad \Leftrightarrow \quad H_{P^{*}((a, 1,1), 0), C_{L^{2}}^{*}}\left(u_{0}, y_{0}\right)^{T} \approx 0,
$$

see [4]. This precludes the direct application of the robust stability theory of [8]. This is a generic problem for closed loops with non-zero responses to zero disturbances or non-continuous behaviour at this point. This arises in a variety of situations; some examples are:

- Smooth adaptive controllers when applied to unstable plants see [4].

- Memoryless feedback designs such as example 5 in [8], when applied to systems with non-zero initial conditions. See for example [7] for an approach to such cases.

Finally note that the adaptive problem concerns the analysis of a controller on a parameterised set of nominal plants (ie. parameterised by the uncertain parameter $\theta$, and also typically the initial condition $\left.y_{1}^{0}\right)$. However the standard gap framework applies to a single fixed nominal plant $P$. The approach taken in this paper is to view the uncertain parameters themselves as inputs to the plant. This has the effect of replacing a linear plant by a nonlinear plant with extra input channels, but has the important advantage of needing only to study a single nominal plant.

In general, suppose the nominal plant is parameterised by $p \in \Pi$ for some appropriate choice of Euclidean space $\Pi$. We then augment the $\mathcal{U}$ disturbance channel to:

$$
\widetilde{\mathcal{U}}:=\widetilde{\Pi} \times L^{2}
$$

where $\widetilde{\Pi}$ denotes the set of constant maps $[0, \infty) \rightarrow \Pi$ ie.

$$
\widetilde{\Pi}=\{f:[0, \infty) \rightarrow \Pi \mid \exists p \in \Pi \text { s.t. } f(t)=p \forall t \in[0, \infty)\} .
$$

Since $\Pi$ and $\widetilde{\Pi}$ are naturally isometrically isomorphic, henceforth we always implicitly make the natural identifications between $\Pi$ and $\widetilde{\Pi}$ and also write $\Pi$ for $\widetilde{\Pi}$.

The plant and controller equations are then redefined appropriately with respect to the new domains and co-domains (see e.g. Section 4.3 for an explicit example). In particular the controller equations are chosen to assign 0 to the $\Pi$ channel, to ensure the nonlinear projection properties of the parallel projection hold. The framework of [8] then applies directly. This idea allows us to consider system responses to parameter variations in the plant eg. $\Pi=\mathbb{R}^{p}$ where $p$ is the dimension of the parameter space. We will return to the problem of non-zero initial conditions in Section 5.3. 


\subsection{The closed loop is gf-stable}

For $1 \leq p \leq \infty$ and $n \geq 1$ we define augmented signal spaces as follows:

$$
\begin{aligned}
u_{i}=\left(\theta, u_{i}^{*}\right)^{T} & \in \widetilde{\mathcal{U}}:=E_{n} \times L^{p}[0, \infty), \\
y_{i} & \in \mathcal{Y}:=L^{p}[0, \infty), \quad i=0,1,2,
\end{aligned}
$$

where the $\widetilde{\mathcal{U}}$ norm is taken to be

$$
\left\|\left(\theta, u_{0}^{*}\right)^{T}\right\|=\sqrt{|\theta|^{2}+\left\|u_{0}^{*}\right\|_{L^{p}[0, \infty)}^{2}} .
$$

Now we return to the concrete example, and establish gain function stability of the augmented closed loop when the initial conditions are zero. Let $p=2$ and $n=1$, and let $\theta=(a, 1,1) \in E_{1}=\mathbb{R}^{3}$. We define the plant as:

$$
\begin{aligned}
& P \quad: \quad\left(E_{n} \times L^{p}[0, \infty)\right)_{a} \rightarrow\left(L^{p}[0, \infty)\right)_{a} \\
& P\left(\theta, u_{1}^{*}\right)=y_{1} \quad: \quad \dot{y}_{1}=a y_{1}+u_{1}^{*}, \quad y_{1}(0)=0,
\end{aligned}
$$

where note that $P$ is not a linear operator. The controller is defined formally as:

$$
\begin{aligned}
C & :\left(L^{p}[0, \infty)\right)_{a} \rightarrow\left(E_{n} \times L^{p}[0, \infty)\right)_{a} \\
C\left(y_{2}\right)=\left(0, u_{2}^{*}\right) \quad: \quad u_{2}^{*} & =-\hat{k} y_{2}-y_{2}, \\
\dot{\hat{k}} & =\alpha \frac{1}{4 \hat{k}^{3}} y_{2}^{2}, \quad \hat{k}(0)=0
\end{aligned}
$$

Note that

$$
P\left(\theta, u_{1}^{*}\right)=P^{*}(\theta, 0)\left(u_{1}^{*}\right)
$$

and

$$
C\left(y_{2}\right)=\left(0, C_{L^{p}}^{*}\left(y_{2}\right)\right)
$$

We now come to the key result:

Proposition 4.3. Let $\mathcal{U}=\mathcal{Y}=L^{2}[0, \infty)$ be defined by (45). Consider $P, C$ defined by (47), (48) and let $\Omega=\mathbb{R} \times\{1\} \times\{1\}$. Then the operator $\left.H_{P, C}\right|_{\Omega \times \mathcal{W}}$ is gain function stable.

Proof. The gain function $\gamma^{*}:[\sqrt{2}, \infty) \rightarrow[0, \infty)$ is defined:

$$
\gamma^{*}(r)=\sup \left\{\left\|T_{\tau} \Pi_{\mathcal{M} / \mathcal{N}} x\right\|:\left\|T_{\tau} x\right\| \leq r\right\}
$$

where

$$
x=\left(u_{0}, y_{0}\right)=\left((a, 1,1), u_{0}^{*}, y_{0}\right)^{T} \in \mathbb{R}^{3} \times L^{2}[0, \infty) \times L^{2}[0, \infty),
$$

so,

$$
\gamma^{*}(r) \leq \sup \left\{\left\|\Pi_{\mathcal{M} / \mathcal{N}}\left((a, 1,1), u_{0}^{*}, y_{0}\right)\right\|: u_{0}^{*}, y_{0} \in L^{2}, \theta \in \mathbb{R}^{3}, a^{2}+2+\left\|u_{0}^{*}\right\|_{L^{2}}^{2}+\left\|y_{0}\right\|_{L^{2}}^{2} \leq r^{2}\right\} .
$$

To establish gf-stability, we consider the bounds (32), (39) of Proposition 4.2 to obtain:

$$
\begin{gathered}
\left\|y_{1}\right\|_{L^{2}\left[t^{*}, \infty\right)}^{3} \leq \quad\left(6 \sqrt{3 \alpha}\left\|y_{0}\right\|_{L^{2}[0, \infty)}\right)\left\|y_{1}\right\|_{L^{2}\left[t^{*}, \infty\right)}^{2}+ \\
\left(6\left(1+|a|^{2}\right)\left\|y_{0}\right\|_{L^{2}[0, \infty)}^{2}+2\left\|u_{0}^{*}\right\|_{L^{2}[0, \infty)}^{2}+\right. \\
\left.4|a|\left(\frac{1}{\sqrt{\alpha}} \theta^{2}+\left\|y_{0}\right\|_{L^{2}[0, \infty)}\right)^{2}+6\left\|y_{0}\right\|_{L^{2}[0, \infty)}^{2}+2\left\|u_{0}^{*}\right\|_{L^{2}[0, \infty)}^{2}\right)\left\|y_{1}\right\|_{L^{2}\left[t^{*}, \infty\right)}+ \\
\sqrt{\frac{3}{\alpha}}\left(a^{4}+3 \alpha\left\|y_{0}\right\|_{L^{2}[0, \infty)}^{2}\right)\left\|y_{0}\right\|_{L^{2}[0, \infty)}^{2}
\end{gathered}
$$


which yields the cubic inequality:

$$
\begin{aligned}
& \left\|y_{1}\right\|_{L^{2}\left[t^{*}, \infty\right)}^{3}-6 \sqrt{3 \alpha} r\left\|y_{1}\right\|_{L^{2}\left[t^{*}, \infty\right)}^{2}- \\
& \quad\left(\frac{4}{\alpha} r^{5}+\left(\frac{8}{\sqrt{\alpha}}+6\right) r^{4}+4 r^{3}+16 r^{2}\right)\left\|y_{1}\right\|_{L^{2}\left[t^{*}, \infty\right)}-\sqrt{\frac{3}{\alpha}}\left(r^{6}+3 \alpha r^{4}\right) \leq 0 .
\end{aligned}
$$

At equality, the above equation has a positive root, since the cubic coefficient is positive and the other coefficients are negative. The minimal positive root is then a bound on $\left\|y_{1}\right\|_{L^{2}\left[t^{*}, \infty\right)}$. Since roots of polynomial equations depend continuously on their coefficients, there is a continuous function $\lambda:[\sqrt{2}, \infty) \rightarrow[0, \infty)$ for which

$$
\left\|y_{1}\right\|_{L^{2}\left[t^{*}, \infty\right)} \leq \lambda(r) .^{3}
$$

Inequality (28) implies:

$$
\left\|y_{1}\right\|_{L^{2}\left[0, t^{*}\right]} \leq \frac{1}{\sqrt{\alpha}} r^{2}+r
$$

Hence,

$$
\left\|y_{1}\right\|_{L^{2}[0, \infty]}=\sqrt{\left\|y_{1}\right\|_{L^{2}\left[0, t^{*}\right]}^{2}+\left\|y_{1}\right\|_{L^{2}\left[t^{*}, \infty\right)}^{2}} \leq \sqrt{\frac{1}{\alpha} r^{4}+\frac{2}{\sqrt{\alpha}} r^{3}+r^{2}+\lambda^{2}(r)}:=\zeta(r) .
$$

Likewise, inequality (41) yields:

$$
\left\|u_{1}\right\|_{L^{2}[0, \infty)}=\left(a^{2}+\left\|u_{1}^{*}\right\|_{L^{2}[0, \infty)}^{2}\right)^{\frac{1}{2}} \leq\left(r^{2}+\left(2 r+\alpha^{\frac{1}{4}}(r+\zeta(r))^{\frac{3}{2}}+\zeta(r)\right)^{2}\right)^{\frac{1}{2}} .
$$

This establishes gain function stability with $\gamma$ taken explicitly as:

$$
\gamma(r)=\left(\frac{1}{\alpha} r^{4}+\frac{2}{\sqrt{\alpha}} r^{3}+2 r^{2}+\lambda^{2}(r)+\left(2 r+\alpha^{\frac{1}{4}}(r+\zeta(r))^{\frac{3}{2}}+\zeta(r)\right)^{2}\right)^{\frac{1}{2}} .
$$

\section{$5 \quad$ Robust stability results}

The three results in this section form the theoretical basis for deducing robustness results from gain function bound we have obtained in Proposition 4.3. Whilst we have considered an $L^{2}$ setting to date, it should be observed that Theorem 5.1 holds in a general signal space setting, whilst Theorems 5.2 and 5.3 hold in any $L^{p}$ space, $1 \leq p \leq \infty$. We will subsequently apply these results in both $L^{2}$ and $L^{\infty}$ settings.

\subsection{A general result}

For our $L^{2}$ example, $\left[P^{*}((a, 1,1), 0), C_{L^{2}}^{*}\right]$, we have demonstrated gain function stability of the augmented closed loop $[P, C]$, hence the global gain function results of [8] can be applied to deduce some form of robust stability for the augmented system $[P, C]$. However, our goal is to interpret the resulting robustness margin in terms of the classical gap metric applied to the nominal (un-augmented) plant $P^{*}((a, 1,1), 0)$ : in particular to elucidate the semi-global nature of the resulting stability under perturbation. For this, we first establish a variant on the gain function stability result of [8]. This result is distinguished from the global gain function result of [8] by the need to consider gain functions over bounded subsets of the graphs, in preparation for the semi-global results.

\footnotetext{
${ }^{3}$ Note that as the inequality is cubic, this function could be computed explicitly.
} 
Theorem 5.1. Let $\mathcal{U}, \mathcal{Y}$ be signal spaces, and let $\mathcal{W}=\mathcal{U} \times \mathcal{Y}$. Suppose $[P, C]$ is gf-stable and $\left[P_{1}, C\right]$ is regularly well-posed. Let $\mathcal{D} \subset \mathcal{G}_{P}, \mathcal{D}_{1} \subset \mathcal{G}_{P_{1}}, \mathcal{X} \subset \mathcal{W}$ and let $r>0$. Suppose $\Pi_{P / / C} \mathcal{X} \subset \mathcal{D}$ and there exists a causal, gf-stable mapping $\Psi: \mathcal{D} \rightarrow \mathcal{D}_{1}$ such that

(i) $T_{\tau}(I-\Psi) \Pi_{P / / C}: \mathcal{X} \rightarrow \mathcal{W}$ is causal and compact for all $\tau>0$.

(ii) $T_{\tau} w+T_{\tau}(I-\Psi) \Pi_{P / / C} x \in \mathcal{X}$ for all $x, w \in \mathcal{X}, \tau>0$.

(iii) There exists a function $\epsilon(\cdot) \in \mathcal{K}_{\infty}$ such that

$$
\left\|(I-\Psi) \Pi_{P / / C} x\right\|_{\tau} \leq(1+\epsilon)^{-1}(r), \quad \forall x \in \mathcal{X},\|x\| \leq r, \tau>0 .
$$

Then $\left.H_{P_{1}, C}\right|_{\mathcal{X} \cap B_{r}}: \mathcal{X} \cap B_{r} \rightarrow \mathcal{W} \times \mathcal{W}$ is gf-stable and

$$
\left\|\Pi_{P_{1} / / C} w\right\|_{\tau} \leq g[\Psi] \circ g\left[\Pi_{P / / C}\right] \circ\left(1+\epsilon^{-1}\right)(r) \quad \forall w \in \mathcal{X},\|w\| \leq r, \tau \geq 0 .
$$

Proof. Let $w \in \mathcal{X},\|w\| \leq r$, and let $\left[0, \omega_{w}\right)$ be the maximal interval of existence for $H_{P_{1}, C} w$. Let $\omega_{w}>\tau>0$. Consider the equation

$$
\begin{aligned}
T_{\tau} w & =T_{\tau}\left(I+(\Psi-I) \Pi_{P / / C}\right) x \\
& =T_{\tau}\left(\Pi_{C / / P}+\Psi \Pi_{P / / C}\right) x .
\end{aligned}
$$

We claim that this equation has a solution $x \in V$ where:

$$
V=\left\{x \in \mathcal{X}:\|x\| \leq\left(1+\epsilon^{-1}\right)(r), \exists y \in \mathcal{W} \text { s.t. } x=T_{\tau} y\right\} .
$$

Consider the operator

$$
Q_{w}: V \rightarrow \mathcal{X} \quad: \quad x \mapsto T_{\tau} w+T_{\tau}(I-\Psi) \Pi_{P / / C} x
$$

where observe, by (ii), that $Q_{w}(V) \subset \mathcal{X}$ as required. By (iii) there exists $\epsilon \in \mathcal{K}_{\infty}$ such that, for all $x \in V$,

$$
\begin{aligned}
\left\|Q_{w} x\right\| & =\left\|T_{\tau} w+T_{\tau}(I-\Psi) \Pi_{P / / C} x\right\| \\
& \leq\|w\|_{\tau}+\left\|(I-\Psi) \Pi_{P / / C} x\right\|_{\tau} \\
& \leq\|w\|_{\tau}+(1+\epsilon)^{-1}\left(\|x\|_{\tau}\right), \\
& \leq r+(1+\epsilon)^{-1} \circ\left(1+\epsilon^{-1}\right)(r), \\
& \leq\left(1+\epsilon^{-1}\right)(r),
\end{aligned}
$$

where the fifth inequality follows from the identity:

$$
r+(1+\epsilon)^{-1} \circ\left(1+\epsilon^{-1}\right)(r)=r+(1+\epsilon)^{-1} \circ(1+\epsilon) \circ \epsilon^{-1}(r)=\left(1+\epsilon^{-1}\right)(r) .
$$

Therefore $Q_{w}(V) \subset V$. Since by (i), $T_{\tau}(I-\Psi) \Pi_{P / / C}$ is compact, it then follows that $Q_{w}$ is compact. Hence by Schauder's fixed point theorem, $Q_{w}$ has a fixed point in $V$. Hence equation (63) has a solution $x \in V \subset \mathcal{X}$ as claimed.

Since $\Psi \Pi_{P / / C} x \in \mathcal{G}_{P_{1}}, \Pi_{C / / P} x \in \mathcal{G}_{C}$ and $\Psi, \Pi_{P_{1} / / C}, \Pi_{P / / C}, \Pi_{C / / P}$ are causal, it follows from equation (63) that

$$
\begin{aligned}
T_{\tau} \Pi_{P_{1} / / C} w & =T_{\tau} \Pi_{P_{1} / / C} T_{\tau} w \\
& =T_{\tau} \Pi_{P_{1} / / C}\left(T_{\tau} \Pi_{C / / P} x+T_{\tau} \Psi \Pi_{P / / C} x\right) \\
& =T_{\tau} \Pi_{P_{1} / / C}\left(\Pi_{C / / P} x+\Psi \Pi_{P / / C} x\right) \\
& =T_{\tau} \Psi \Pi_{P / / C} x
\end{aligned}
$$


hence since $x \in V$,

$$
\begin{aligned}
\left\|\Pi_{P_{1} / / C} w\right\|_{\tau} & =\left\|\Psi \Pi_{P / / C} x\right\|_{\tau} \\
& \leq g[\Psi] \circ g\left[\Pi_{P / / C}\right]\left(\|x\|_{\tau}\right) \\
& \leq g[\Psi] \circ g\left[\Pi_{P / / C}\right] \circ\left(1+\epsilon^{-1}\right)(r) .
\end{aligned}
$$

As $\mathcal{W}$ has the property that $\sup _{\tau \geq 0}\left\|T_{\tau} x\right\|<\infty$ implies $x \in \mathcal{W}$, and since $\omega_{w}>\tau>0$ was arbitrary it follows that $T_{\omega_{w}} \Pi_{P_{1} / / C} w \in \mathcal{W}$, and so $T_{\omega_{w}} H_{P_{1} / / C} w \in \mathcal{W} \times \mathcal{W}$. Since $\left[P_{1}, C\right]$ is regularly well posed, it follows that $\omega_{w}=\infty$ and $\Pi_{P_{1} / / C} w \in \mathcal{W}$. Since $w \in \mathcal{X} \cap B_{r}$ was arbitrary, it follows that (62) holds and hence $H_{P_{1}, C}: \mathcal{X} \cap B_{r} \rightarrow \mathcal{W} \times \mathcal{W}$ is gf-stable as required. This completes the proof.

\subsection{Robustness margins for the non-augmented closed loop}

A combination of Proposition 4.3 and Theorem 5.1 will establish a form of robust stability for the augmented closed loop system. The first result of this section relates this robust stability of the augmented closed loop to the robustness of the un-augmented nominal plant with zero initial conditions with respect to sufficiently small perturbations as measured by the gap metric.

Theorem 5.2. Let $p \in[1, \infty], n, q \in \mathbb{N}, \mathcal{U}=L^{p}[0, \infty), \mathcal{Y}=L^{p}[0, \infty)$ and $\mathcal{W}=\mathcal{U} \times \mathcal{Y}$. Let $K^{*}: \mathcal{Y}_{a} \rightarrow \mathcal{U}_{a}$ be causal, consider $P^{*}(\vartheta, 0): \mathcal{U}_{a} \rightarrow \mathcal{Y}_{a}$ defined in $(8)$ for $\left(\vartheta, x^{0}\right)$ in $\mathcal{L}_{q} \times \mathbb{R}^{q}$ or $\mathcal{L}_{n} \times \mathbb{R}^{n}$, and suppose $\left[P^{*}(\vartheta, 0), K^{*}\right]$ is regularly well posed for all $\vartheta \in \mathcal{L}_{q}$. Define

$$
\begin{array}{rrr}
P: E_{n} \times \mathcal{U}_{a} \rightarrow \mathcal{Y}_{a}, & \left(\vartheta, u_{1}\right) \mapsto P\left(\vartheta, u_{1}\right) & =P^{*}(\vartheta, 0)\left(u_{1}\right) \\
C: \mathcal{Y}_{a} \rightarrow E_{n} \times \mathcal{U}_{a}, & y_{2} \mapsto C\left(y_{2}\right) & =\left(0, K^{*}\left(y_{2}\right)\right)^{T} .
\end{array}
$$

Let $\Omega \subset E_{n}$ be closed. Suppose $\left.H_{P, C}\right|_{\Omega \times \mathcal{W}}$ is gf-stable and $T_{\tau} \Pi_{P / / C}$ is continuous for all $\tau>0$. Then there exists a continuous function $\mu: \mathbb{R}_{+} \times \Omega \rightarrow(0, \infty)$ such that for all $\theta \in \Omega, \theta_{1} \in \mathcal{L}_{q}, w_{0} \in \mathcal{W}$, $\left\|w_{0}\right\| \leq r$

$$
\vec{\delta}\left(P^{*}(\theta, 0), P^{*}\left(\theta_{1}, 0\right)\right) \leq \mu(r, \theta) \Rightarrow H_{P^{*}\left(\theta_{1}, 0\right), K^{*}} w_{0} \in \mathcal{W} \times \mathcal{W} .
$$

Proof. Let $\epsilon \in \mathcal{K}_{\infty}, 0<\nu<1$ and let $r_{0}=\inf _{\vartheta \in \Omega}|\vartheta|$. Since $[P, C]$ is gf-stable, the gain function $g\left[\left.\Pi_{P / / C}\right|_{\Omega \times \mathcal{W}}\right]:\left(r_{0}, \infty\right) \rightarrow[0, \infty)$ is defined. As $g\left[\left.\Pi_{P / / C}\right|_{\Omega \times \mathcal{W}}\right]$ is monotonically increasing, there exists a continuous function $\gamma:\left(r_{0}, \infty\right) \rightarrow[0, \infty)$ s.t. $\gamma(\alpha) \geq g\left[\left.\Pi_{P / / C}\right|_{\Omega \times \mathcal{W}}\right](\alpha)$ for all $\alpha>r_{0}$. Define continuous functions $\beta:\left(r_{0}, \infty\right) \rightarrow(0, \infty)$ and $\mu: \mathbb{R}_{+} \times \Omega \rightarrow(0, \infty)$ by

$$
\begin{aligned}
\beta(r) & =\min \left\{1-\nu, \inf _{r_{0} \leq \alpha \leq r} \frac{(1+\epsilon)^{-1}(\alpha)}{2 \gamma(\alpha)}\right\}, \\
\mu\left(r_{1}, \vartheta\right) & =\beta\left(\sqrt{r_{1}^{2}+|\vartheta|^{2}}\right) .
\end{aligned}
$$

Observe that $\beta(r)=0$ if and only if $\frac{(1+\epsilon)^{-1}\left(r_{0}\right)}{2 \gamma\left(r_{0}\right)}=0$, which implies $r_{0}=0$. But if $r_{0}=0$, then by the definition of $r_{0}$, and since $\Omega$ is closed, we have $0 \in \Omega \subset \mathcal{L}_{n}$. This is a contradiction, so $r_{0}>0$.

Let $w_{0}=\left(u_{0}, y_{0}\right)^{T} \in \mathcal{W}, \theta \in \Omega \subset \mathcal{L}_{n}, \theta_{1} \in \mathcal{L}_{q}$ be such that

$$
\delta\left(P^{*}(\theta, 0), P^{*}\left(\theta_{1}, 0\right)\right) \leq \mu\left(\left\|w_{0}\right\|, \theta\right) .
$$

Let $r^{2}=2\left(\left\|w_{0}\right\|^{2}+|\theta|^{2}\right)>0$, hence

$$
\delta\left(P^{*}(\theta, 0), P^{*}\left(\theta_{1}, 0\right)\right) \leq \beta(r) .
$$


We need to show $\left(w_{1}, w_{2}\right)=H_{P^{*}\left(\theta_{1}, 0\right), K^{*}}\left(w_{0}\right) \in \mathcal{W} \times \mathcal{W}$.

To apply Theorem 5.1, with the augmented signal space $E_{n} \times \mathcal{W}$ for $\mathcal{W}$, we define

$$
P_{1}: E_{n} \times \mathcal{U}_{a} \rightarrow \mathcal{Y}_{a}, \quad P_{1}\left(\theta_{1}, u_{1}\right)=P^{*}(\theta, 0)\left(u_{1}\right) .
$$

Since $\left[P^{*}\left(\theta_{1}, 0\right), K^{*}\right]$ is regularly well posed, it follows that $\left[P_{1}, C\right]$ is regularly well posed.

Let

$$
\mathcal{D}=\left\{\left(\begin{array}{c}
\vartheta \\
w
\end{array}\right) \in E_{n} \times \mathcal{W}: w \in \mathcal{G}_{P^{*}(\theta, 0)}, \vartheta=\theta\right\} \subset \mathcal{G}_{P},
$$

and

$$
\mathcal{D}_{1}=\left\{\left(\begin{array}{c}
\vartheta \\
w
\end{array}\right) \in E_{n} \times \mathcal{W}: w \in \mathcal{G}_{P^{*}\left(\theta_{1}, 0\right)}, \vartheta=\theta\right\} \subset \mathcal{G}_{P_{1}} .
$$

Let

$$
\mathcal{X}=\left\{\left(\begin{array}{c}
\vartheta \\
w
\end{array}\right) \in E_{n} \times \mathcal{W}: \vartheta=\theta\right\} \subset \Omega \times \mathcal{W} .
$$

We now verify the assumptions of Theorem 5.1. By the definition of $P, C$ and since $\left.\Pi_{P / / C}\right|_{\Omega \times \mathcal{W}}$ is gf-stable, it follows that $\Pi_{P / / C} \mathcal{X} \subset \mathcal{D}$.

We now construct a mapping $\Psi: \mathcal{D} \rightarrow \mathcal{D}_{1}$ with the properties required by Theorem 5.1. First note that

$$
\vec{\delta}\left(P^{*}(\theta, 0), P^{*}\left(\theta_{1}, 0\right)\right)=\inf _{\Phi \in \mathcal{O}} \sup _{w \in \mathcal{G}_{P^{*}(\theta, 0)} \backslash\{0\}, \tau>0} \frac{\left\|\left.(I-\Phi)\right|_{\mathcal{G}_{P^{*}(\theta, 0)}} w\right\|_{\tau}}{\|w\|_{\tau}},
$$

where

$$
\mathcal{O}=\left\{\Phi: \mathcal{G}_{P^{*}(\theta, 0)} \rightarrow \mathcal{G}_{P^{*}\left(\theta_{1}, 0\right)}: \text { is causal, bijective and } \Phi(0)=0\right\} .
$$

Hence there exists a mapping $\Phi_{\theta} \in \mathcal{O}$ such that,

$$
2 \vec{\delta}\left(P^{*}(\theta, 0), P^{*}\left(\theta_{1}, 0\right)\right) \geq \sup _{w \in \mathcal{G}_{P^{*}(\theta, 0)} \backslash\{0\}, \tau>0} \frac{\left\|\left.\left(I-\Phi_{\theta}\right)\right|_{\mathcal{G}_{P^{*}(\theta, 0)}} w\right\|_{\tau}}{\|w\|_{\tau}} .
$$

Furthermore, $\Phi_{\theta}$ can always be chosen to make $\mathbf{L}\left(I-\Phi_{\theta}\right) \in \mathcal{R}$ (in the frequency domain) and strictly proper (see the proof of Proposition 5, [8]), hence $T_{\tau}\left(I-\Phi_{\theta}\right)$ is compact for all $0<\tau<\infty$.

Define the mapping $\Psi: \mathcal{D} \rightarrow \mathcal{D}_{1}$ by

$$
\Psi\left(\begin{array}{c}
\theta \\
w
\end{array}\right)=\left(\begin{array}{c}
\theta \\
\Phi_{\theta}(w)
\end{array}\right)
$$

We first establish condition (i) of Theorem 5.1. Since the mapping $\Psi$ is causal by the causality of $\Phi_{\theta}$, and since $\left.\Pi_{P / / C}\right|_{\Omega \times \mathcal{W}}$ is gf-stable, hence causal, it follows that $T_{\tau}(I-\Psi) \Pi_{P / / C}: \mathcal{X} \rightarrow \mathcal{W}$ is causal for all $0<\tau<\infty . T_{\tau}\left(I-\Phi_{\theta}\right)$ is continuous hence $T_{\tau}(I-\Psi)$ is continuous for all $0<\tau<\infty$. Since $T_{\tau} \Pi_{P / / C}$ is continuous for all $0<\tau<\infty$ and $\Psi$ is causal, it follows that $T_{\tau}(I-\Psi) \Pi_{P / / C}=T_{\tau}(I-\Psi) T_{\tau} \Pi_{P / / C}$ is continuous for all $0<\tau<\infty$.

As $\left.\Pi_{P / / C}\right|_{\Omega \times \mathcal{W}}$ is gf-stable, $\left.T_{\tau}\left(I-\Phi_{\theta}\right)\right|_{\mathcal{G}_{P^{*}(\theta, 0)}}$ is compact, and

$$
\left.(I-\Psi)\right|_{\mathcal{D}}\left(\begin{array}{c}
\theta \\
w
\end{array}\right)=\left(\begin{array}{c}
0 \\
\left(I-\Phi_{\theta}\right)(w)
\end{array}\right), \quad \forall\left(\begin{array}{c}
\theta \\
w
\end{array}\right) \in \mathcal{D},
$$

it follows that $T_{\tau}(I-\Psi) \Pi_{P / / C}: \mathcal{X} \rightarrow \mathcal{W}$ maps bounded sets into relatively compact sets for all $\tau>0$. Hence $T_{\tau}(I-\Psi) \Pi_{P / / C}: \mathcal{X} \rightarrow \mathcal{W}$ is compact for all $\tau>0$ as required. 
Condition (ii) of Theorem 5.1 follows from the fact that for all $x, w \in \mathcal{X}$,

$$
T_{\tau} w+T_{\tau}(I-\Psi) \Pi_{P / / C} x=T_{\tau}\left(\begin{array}{c}
\theta \\
w^{\prime}
\end{array}\right) \in \mathcal{X} \quad \text { for some } \quad w^{\prime} \in \mathcal{W} .
$$

We now verify the condition (61) of Theorem 5.1 to establish condition (iii). First we establish a key inequality:

$$
\begin{aligned}
2 \vec{\delta}\left(P^{*}(\theta, 0), P^{*}\left(\theta_{1}, 0\right)\right) & \geq \sup _{w \in \mathcal{G}_{P^{*}(\theta, 0)} \backslash\{0\}, \tau>0} \frac{\left\|\left.\left(I-\Phi_{\theta}\right)\right|_{\mathcal{G}_{P^{*}(\theta, 0)}} w\right\|_{\tau}}{\|w\|_{\tau}} \\
& \geq \sup _{(\theta, w) \in \mathcal{D} \backslash\{0\}, \tau>0} \frac{\left\|\left.(I-\Psi)\right|_{\mathcal{D}}\left(\theta, w^{T}\right)^{T}\right\|_{\tau}}{\left\|\left(\theta, w^{T}\right)^{T}\right\|_{\tau}}
\end{aligned}
$$

where the second inequality follows from the equation (81).

Now let $\tau>0$ and $x \in \mathcal{X},\|x\| \leq r$. Then since $\Pi_{P / / C} x \in \mathcal{D} \subset \mathcal{G}_{P}$, inequality (83) gives:

$$
\begin{aligned}
\left\|(I-\Psi) \Pi_{P / / C} x\right\|_{\tau} & \leq 2 \vec{\delta}\left(P^{*}(\theta, 0), P^{*}\left(\theta_{1}, 0\right)\right)\left\|\Pi_{P / / C} x\right\|_{\tau} \\
& \leq 2 \vec{\delta}\left(P^{*}(\theta, 0), P^{*}\left(\theta_{1}, 0\right)\right) g\left[\left.\Pi_{P / / C}\right|_{\Omega \times \mathcal{W}}\right]\left(\|x\|_{\tau}\right),
\end{aligned}
$$

where the first line follows from inequality (83). Since $\|x\|_{\tau} \leq r$, it follows from the definition of $\beta$ and inequality (72) that:

$$
\begin{aligned}
\vec{\delta}\left(P^{*}(\theta, 0), P^{*}\left(\theta_{1}, 0\right)\right) g\left[\left.\Pi_{P / / C}\right|_{\Omega \times \mathcal{W}}\right]\left(\|x\|_{\tau}\right) & \leq \beta(r) g\left[\left.\Pi_{P / / C}\right|_{\Omega \times \mathcal{W}}\right]\left(\|x\|_{\tau}\right) \\
& \leq \beta\left(\|x\|_{\tau}\right) g\left[\left.\Pi_{P / / C}\right|_{\Omega \times \mathcal{W}}\right]\left(\|x\|_{\tau}\right) \\
& \leq \beta\left(\|x\|_{\tau}\right) \gamma\left(\|x\|_{\tau}\right) \\
& \leq \frac{1}{2}(1+\epsilon)^{-1}\left(\|x\|_{\tau}\right) .
\end{aligned}
$$

Hence by inequalities (84), (85), which hold for all $x \in \mathcal{X},\|x\| \leq r$ and $\tau>0$, we have established the inequality (61), and hence condition (iii), as required.

Hence it follows from Theorem 5.1 that $\left.H_{P_{1}, C}\right|_{\mathcal{X} \cap B_{r}}$ is gf-stable and equation (62) holds. In particular, since $\left(\begin{array}{c}\theta \\ w_{0}\end{array}\right) \in \mathcal{X},\left(\begin{array}{c}\theta \\ w_{0}\end{array}\right) \in B_{r}$ this implies $H_{P_{1}, C}\left(\begin{array}{c}\theta \\ w_{0}\end{array}\right) \in E_{n} \times \mathcal{W} \times E_{n} \times \mathcal{W}$, and consequently $H_{P^{*}\left(\theta_{1}, 0\right), K^{*}} w_{0} \in \mathcal{W} \times \mathcal{W}$. Since $\theta \in \Omega, w_{0} \in \mathcal{W}$ were arbitrary, the result follows.

\subsection{Incorporation of initial conditions}

The third result in this section then shows that a robust stability guarantee for a closed loop free of initial conditions also guarantees a stability margin in the presence of sufficiently small initial conditions. We emphasise that the following result makes no restriction other than causality on the (nonlinear) controller $K^{*}$.

Theorem 5.3. Let $p \in[1, \infty], n, q \in \mathbb{N}, \mathcal{U}=L^{p}[0, \infty), \mathcal{Y}=L^{p}[0, \infty)$ and $\mathcal{W}=\mathcal{U} \times \mathcal{Y}$. Let $K^{*}: \mathcal{Y}_{a} \rightarrow \mathcal{U}_{a}$ be causal, and consider $P^{*}(\theta, 0): \mathcal{U}_{a} \rightarrow \mathcal{Y}_{a}$ defined in $(8)$ where $(\theta, 0) \in \mathcal{L}_{n} \times \mathbb{R}^{n}$. Suppose there exists $r>0$ such that $H_{P^{*}(\theta, 0), K^{*}} \in \mathcal{W} \times \mathcal{W}$ for all $w_{0} \in \mathcal{W},\left\|w_{0}\right\| \leq r$. Then there exists $\lambda>0$ such that $H_{P^{*}\left(\theta, x_{1}^{0}\right), K^{*}} \in \mathcal{W} \times \mathcal{W}$ for all $\left(\theta, x_{1}^{0}\right) \in \mathcal{L}_{n} \times \mathbb{R}^{n}$ such that $\lambda\left|x_{1}^{0}\right|+\left\|w_{0}\right\| \leq r$ 
Proof. Let $\theta_{1}=(A, B, C) \in \mathcal{L}_{q}, x_{1}^{0} \in \mathbb{R}^{q}$. We first characterize the graph $\mathcal{G}_{P^{*}\left(\theta_{1}, x_{1}^{0}\right)}$.

Let $F \in \mathbb{R}^{m \times q}$ be such that $\hat{A}=A+B F$ is Hurwitz (note that a suitable $F$ exists since the system is $P^{*}\left(\theta_{1}, 0\right)$ is stabilizable). Define $N: \mathcal{U} \rightarrow N(\mathcal{U}), v \mapsto u, M: \mathcal{U} \rightarrow \mathcal{Y}, v \mapsto y$, where

$$
\begin{aligned}
\dot{x} & =(A+B F) x+B v, \quad x(0)=0 \\
u & =F x+v \\
y & =C x
\end{aligned}
$$

Observe that $N(\mathcal{U})=V:=\left\{u \in \mathcal{U}: P^{*}\left(\theta_{1}, 0\right) u \in \mathcal{Y}\right\}, N: \mathcal{U} \rightarrow V$ is invertible and $P^{*}\left(\theta_{1}, 0\right)=M N^{-1}$. Let

$$
q_{v}:=\left(\begin{array}{c}
N \\
M
\end{array}\right) v+\left(\begin{array}{c}
F \exp (\hat{A} \cdot) x_{1}^{0} \\
C \exp (\hat{A} \cdot) x_{1}^{0}
\end{array}\right) .
$$

We claim $\mathcal{G}_{P^{*}\left(\theta_{1}, x_{1}^{0}\right)}=Q:=\left\{q_{v} \in \mathcal{W}: v \in \mathcal{U}\right\}$.

Consider any $q_{v} \in Q, v \in \mathcal{U}$. Let $u=N v+F \exp (\hat{A} \cdot) x_{1}^{0}$. Since $N v \in \mathcal{U}, M v \in \mathcal{Y}$ and $\exp (\hat{A} \cdot) \in$ $L^{p}\left[\mathbb{R}, \mathbb{R}^{m}\right]=\mathcal{Y}$, we have $u \in \mathcal{U}$ and,

$$
\begin{aligned}
P^{*}\left(\theta_{1}, x_{1}^{0}\right) u & =P^{*}\left(\theta_{1}, 0\right) N v+P^{*}\left(\theta_{1}, x_{1}^{0}\right)\left(F \exp (\hat{A} \cdot) x_{1}^{0}\right) \\
& =M(N)^{-1} N v+C \exp (\hat{A} \cdot) x_{1}^{0} \\
& =M v+C \exp (\hat{A} \cdot) x_{1}^{0} \in \mathcal{Y}
\end{aligned}
$$

Therefore $q_{v}=\left(u, P^{*}\left(\theta_{1}, x_{1}^{0}\right) u\right)^{T} \in \mathcal{U} \times \mathcal{Y}$, so $q_{v} \in \mathcal{G}_{P^{*}\left(\theta_{1}, x_{1}^{0}\right)}$ and hence $Q \subseteq \mathcal{G}_{P^{*}\left(\theta_{1}, x_{1}^{0}\right)}$.

Conversely suppose $\left(u, P^{*}\left(\theta_{1}, x_{1}^{0}\right) u\right)^{T} \in \mathcal{G}_{P^{*}\left(\theta_{1}, x_{1}^{0}\right)}$. Then

$$
P^{*}\left(\theta_{1}, 0\right)\left(u-F \exp (\hat{A} \cdot) x_{1}^{0}\right)=P^{*}\left(\theta_{1}, x_{1}^{0}\right) u-P^{*}\left(\theta_{1}, x_{1}^{0}\right)\left(F \exp (\hat{A} \cdot) x_{1}^{0}\right)
$$

and since the right hand side lies in $\mathcal{Y}$, it follows that $P^{*}\left(\theta_{1}, 0\right)\left(u-F \exp (\hat{A} \cdot) x_{1}^{0}\right) \in \mathcal{Y}$. Therefore $u-F \exp (\hat{A} \cdot) x_{1}^{0} \in V=\operatorname{im}(N)$, and so there exists $v \in \mathcal{U}$ s.t. $N v=u-F \exp (\hat{A} \cdot) x_{1}^{0}$. Therefore equation (88) holds, hence

$$
\left(\begin{array}{c}
u \\
P^{*}\left(\theta_{1}, x_{1}^{0}\right) u
\end{array}\right)=q_{v} \in Q
$$

and so $\mathcal{G}_{P^{*}\left(\theta_{1}, x_{1}^{0}\right)} \subseteq Q$. Therefore we have shown $\mathcal{G}_{P^{*}\left(\theta_{1}, x_{1}^{0}\right)}=Q$ as claimed.

Now let

$$
\lambda=\left\|\left(\begin{array}{c}
F \exp (\hat{A} \cdot) \\
C \exp (\hat{A} \cdot)
\end{array}\right)\right\|
$$

and suppose $w_{0} \in \mathcal{W}, x_{1}^{0} \in \mathbb{R}^{q}$ satisfy

$$
\lambda\left|x_{1}^{0}\right|+\left\|w_{0}\right\| \leq r
$$

Then by letting

$$
w_{0}^{\prime}=w_{0}-w_{0}^{\prime \prime}, \quad w_{0}^{\prime \prime}=\left(\begin{array}{c}
F \exp (\hat{A} \cdot) x_{1}^{0} \\
C \exp (\hat{A} \cdot) x_{1}^{0}
\end{array}\right)
$$

we have

$$
\left\|w_{0}^{\prime}\right\| \leq \lambda\left|x_{1}^{0}\right|+\left\|w_{0}\right\| \leq r
$$

hence

$$
H_{P^{*}\left(\theta_{1}, 0\right), K^{*}}\left(w_{0}^{\prime}\right)=\left(w_{1}, w_{2}\right) \in \mathcal{G}_{P^{*}\left(\theta_{1}, 0\right)} \times \mathcal{G}_{K^{*}}
$$


In particular,

$$
w_{0}^{\prime}=w_{1}+w_{2}
$$

and by rearranging we have

$$
w_{0}=\left(w_{1}+w_{0}^{\prime \prime}\right)+w_{2}
$$

Since $w_{1} \in \mathcal{G}_{P^{*}\left(\theta_{1}, 0\right)}$, there exists $v \in \mathcal{U}$ such that $w_{1}=\left(\begin{array}{c}N \\ M\end{array}\right) v$, hence $w_{1}+w_{0}^{\prime \prime} \in Q=\mathcal{G}_{P^{*}\left(\theta_{1}, x_{1}^{0}\right)}$. Since $w_{2} \in \mathcal{G}_{K^{*}}$,

$$
H_{P^{*}\left(\theta_{1}, x_{1}^{0}\right), K^{*}} w_{0}=\left(w_{1}+w_{0}^{\prime \prime}, w_{2}\right) \in \mathcal{G}_{P^{*}\left(\theta_{1}, x_{1}^{0}\right)} \times \mathcal{G}_{K^{*}} \subset \mathcal{W} \times \mathcal{W}
$$

\subsection{Application to the first order $L^{2}$ example}

We now apply the general results of Section 5 to the proof of Theorem 3.1 for our motivating nominal plant $P^{*}\left((a, 1,1), y_{1}^{0}\right)$.

Proof. [Theorem 3.1] We first verify the required properties to apply Theorem 5.2 and Theorem 5.3. Let $p=2$ and note that $K^{*}=C_{L^{2}}^{*}$ is causal. If $\theta_{1} \in \mathcal{L}_{q}$ then $\left[P^{*}\left(\theta_{1}, 0\right), C_{L^{2}}^{*}\right]$ is regularly well posed by Lemma 4.1. Let $a>0$ and let $\Omega=\left\{\theta \in \mathbb{R}^{3} \mid \theta=(a, 1,1), a \in \mathbb{R}\right\} \subset \mathbb{R}^{3}=E_{1}$. Proposition 4.3 establishes gain function stability of $\left.H_{P, C}\right|_{\Omega \times \mathcal{W}}$. Let $\tau>0$. The continuity of $T_{\tau} \Pi_{P / / C}$ follows from the continuity of $T_{\tau} \Pi_{P^{*}(\theta, 0) / / C_{L^{2}}^{*}}$ which in turn follows from the standard results in differential equations (see e.g. the proof of Theorem $4 \mathrm{D}$ in [25]). Therefore the result follows from Theorem 5.2 and Theorem 5.3.

\section{Adaptive controllers in an $L^{\infty}$ setting}

It is very well known that arbitrarily small $L^{\infty}$ disturbances can destabilize closed loop systems containing the nominal scalar plant $P^{*}\left((a, 1,1), y_{1}^{0}\right)$ and adaptive controllers with pure integral actions within the adaptive laws (such as (15)), see eg. [17], [19], [8], [9].

The modifications of the 1980's were designed to overcome these de-stabilising effects. These alterations did not remove the integral action, rather they aimed to prevent the integral action from coming unbounded by a variety of relatively ad-hoc techniques (projections, $\sigma$ modification, dead-zones et.c.). We next demonstrate that these ideas can also be used to modify our $L^{2}$ controller (15) in order that in can operate in the $L^{\infty}$ setting, by considering the projection modification. We also develop an alternative control design providing robustness to $L^{\infty}$ disturbances. At the end of the section, the relative merits of the different approaches are appraised.

Let $\mathcal{U}=\mathcal{Y}=L^{\infty}[0, \infty)$. The first controller considered, $C_{\operatorname{Proj}\left(a_{\max }\right)}^{*}: \mathcal{Y}_{a} \rightarrow \mathcal{U}_{a}$, is the standard projection modification to the $L^{2}$ design defined by:

$$
\begin{aligned}
C_{\operatorname{Proj}\left(a_{\max }\right)}^{*}\left(y_{2}\right): u_{2} & =-\hat{k} y_{2}-y_{2} \\
\dot{\hat{k}}(t) & =\left\{\begin{array}{lll}
\alpha \frac{1}{4 \hat{k}^{3}} y_{2}^{2}, & \text { if } \quad \hat{k}<a_{\max } \\
0, & \text { if } \hat{k} \geq a_{\max } .
\end{array}\right.
\end{aligned}
$$

The second controller considered, $C_{L^{\infty}}^{*}: \mathcal{Y} \rightarrow \mathcal{U}$, is the direct analogue of equation (15) in the $L^{\infty}$ setting:

$$
\begin{aligned}
C_{L^{\infty}}^{*}\left(y_{2}\right): u_{2} & =-\hat{k} y_{2}-y_{2} \\
\hat{k}(t) & =\left(\alpha\left\|y_{2}\right\|_{L^{\infty}[0, t]}\right)^{\frac{1}{2}}
\end{aligned}
$$


We first confirm the property of regular well-posedness for feedback systems containing these controllers and linear plants in $\mathcal{L}$ :

Lemma 6.1. Let $\mathcal{U}=\mathcal{Y}=L^{\infty}[0, \infty)$, suppose $\left(\theta, x_{1}^{0}\right) \in \mathcal{L}_{n} \times \mathbb{R}^{n}$ for some $n \geq 1$ and let $a_{\max }>0$. Then the feedback interconnections $\left[P^{*}\left(\theta, x_{1}^{0}\right), C_{\operatorname{Proj}\left(a_{\max }\right)}^{*}\right]$ and $\left[P^{*}\left(\theta, x_{1}^{0}\right), C_{L^{\infty}}^{*}\right]$ are regularly well posed.

Proof. We first observe that $\left[P^{*}\left(\theta, x_{1}^{0}\right), C_{\mathrm{Proj}\left(a_{\max }\right)}^{*}\right]$ and $\left[P^{*}\left(\theta, x_{1}^{0}\right), C_{L^{\infty}}^{*}\right]$ are locally well posed by standard results on ordinary differential equations. Let us consider either $\left[P^{*}\left(\theta, x_{1}^{0}\right), C_{\operatorname{Proj}\left(a_{\max }\right)}^{*}\right]$ or $\left[P^{*}\left(\theta, x_{1}^{0}\right), C_{L^{\infty}}^{*}\right]$. Suppose $w_{0} \in \mathcal{W}$, and consider $\left(w_{1}, w_{2}\right)=H_{P^{*}(\theta, 0), C_{L}^{*}}\left(w_{0}\right)$ where $\operatorname{dom}\left(w_{1}, w_{2}\right)=[0, \omega)$ is maximal. Suppose $T_{\omega}\left(w_{1}, w_{2}\right) \in \mathcal{W} \times \mathcal{W}$. Then $y_{1} \in L^{\infty}[0, \omega)$. In the case of $\left[P^{*}\left(\theta, x_{1}^{0}\right), C_{L^{\infty}}^{*}\right]$, since $k(t)=\sqrt{\alpha}\left\|y_{2}\right\|_{L^{\infty}[0, t]}^{\frac{1}{2}} \leq \sqrt{\alpha}\left(\left\|y_{0}\right\|_{L^{\infty}[0, t]}+\left\|y_{1}\right\|_{L^{\infty}[0, t]}\right)^{\frac{1}{2}}$ for $0 \leq t<\omega$, it follows that $k$ is uniformly bounded on $[0, \omega)$. In the case of $\left[P^{*}\left(\theta, x_{1}^{0}\right), C_{\operatorname{Proj}\left(a_{\max }\right)}^{*}\right], k$ is uniformly bounded by $a_{\max }$. Thus in both cases the closed loop $x$ sub-system is a linear time-varying system on $[0, \omega)$ with an $L^{\infty}$ input, hence finite escape time is not possible, thus $\omega=\infty$ as required.

\subsection{The main results}

Proposition 6.2. Let $\mathcal{U}=\mathcal{Y}=L^{\infty}[0, \infty)$ and suppose $a, y_{1}^{0} \in \mathbb{R}$. Then

1. The feedback interconnection $\left[P^{*}\left((a, 1,1), y_{1}^{0}\right), C_{L^{\infty}}^{*}\right]$ is BIBO stable.

2. If $a_{\max } \geq a$ then the feedback interconnection $\left[P^{*}\left((a, 1,1), y_{1}^{0}\right), C_{\operatorname{Proj}\left(a_{\max }\right)}^{*}\right.$ is BIBO stable.

Proof. We first establish 1. Let $\left(u_{0}, y_{0}\right)^{T} \in \mathcal{W}$ and let $[0, \omega)$ be the maximal interval of existence. Let $0 \leq t^{*}<\omega$ be defined by $t^{*}=\inf \{t \geq 0: \hat{k}(t) \geq a\}$ when the infimum exists, and $t^{*}=\omega$ otherwise. Then:

$$
\left\|y_{1}\right\|_{L^{\infty}\left[0, t^{*}\right]} \leq\left\|y_{2}\right\|_{L^{\infty}\left[0, t^{*}\right]}+\left\|y_{0}\right\|_{L^{\infty}\left[0, t^{*}\right]}=\frac{1}{\alpha} \hat{k}^{2}\left(t^{*}\right)+\left\|y_{0}\right\|_{L^{\infty}\left[0, t^{*}\right]} \leq \frac{1}{\alpha} a^{2}+\left\|y_{0}\right\|_{L^{\infty}[0, \infty)} .
$$

Now we bound $y_{1}\left(t^{*}\right)$. Define $V: \mathbb{R} \rightarrow \mathbb{R}_{+}$by: $V\left(y_{1}\right)=\frac{1}{2} y_{1}^{2}$. Now as previously,

$$
\dot{V}=(a-\hat{k}-1) y_{1}^{2}+u_{0} y_{1}+y_{0} y_{1}+\hat{k} y_{0} y_{1}
$$

and applying Young's inequality $\left(a b-\frac{1}{4} b^{2} \leq a^{2}\right)$ twice we obtain:

$$
\dot{V} \leq-\frac{1}{2} y_{1}^{2}+3\left(1+\hat{k}^{2}\right) y_{0}^{2}+u_{0}^{2}+(|a|+|\hat{k}|) y_{1}^{2}
$$

Since $\dot{V} \leq 0$ if $y_{1}^{2} \geq 6\left(1+\hat{k}^{2}\right) y_{0}^{2}+2 u_{0}^{2}+2(|a|+|\hat{k}|) y_{1}^{2}$ it follows that

$$
\begin{aligned}
y_{1}^{2}\left(t^{*}\right) & \leq \max \left\{\left(y_{1}^{0}\right)^{2}, 6\left(1+a^{2}\right)\left\|y_{0}\right\|_{L^{\infty}\left[0, t^{*}\right)}^{2}+2\left\|u_{0}\right\|_{L^{\infty}\left[0, t^{*}\right)}^{2}+4|a|\left\|y_{1}\right\|_{L^{\infty}\left[0, t^{*}\right)}^{2}\right\} \\
& \leq \max \left\{\left(y_{1}^{0}\right)^{2}, 6\left(1+a^{2}\right)\left\|y_{0}\right\|_{L^{\infty}[0, \infty)}^{2}+2\left\|u_{0}\right\|_{L^{\infty}[0, \infty)}^{2}+4|a|\left(\frac{1}{\alpha} a^{2}+\left\|y_{0}\right\|_{L^{\infty}[0, \infty)}\right)^{2}\right\}
\end{aligned}
$$

We now consider the $L^{\infty}$ estimates on $\left[t^{*}, \omega\right)$. Since $\hat{k}$ is non-decreasing it follows that $a-\hat{k} \leq 0$ for $t^{*} \leq t<\omega$, hence we can establish an inequality of the form:

$$
\begin{aligned}
\dot{V} & \leq-y_{1}^{2}+u_{0} y_{1}+y_{0} y_{1}+\hat{k} y_{0} y_{1} \\
& \leq-\frac{1}{2} y_{1}^{2}+3\left(1+\hat{k}^{2}\right) y_{0}^{2}+u_{0}^{2} .
\end{aligned}
$$


Since $\dot{V} \leq 0$ if $y_{1}^{2} \geq 6\left(1+\hat{k}^{2}\right) y_{0}^{2}+2 u_{0}^{2}$ we have:

$$
\begin{aligned}
\left\|y_{1}\right\|_{L^{\infty}\left[t^{*}, t\right]}^{2} & \leq \max \left\{y_{1}^{2}\left(t^{*}\right), 6\left(1+|\hat{k}(t)|^{2}\right)\left\|y_{0}\right\|_{L^{\infty}\left[t^{*}, t\right]}^{2}+2\left\|u_{0}\right\|_{L^{\infty}\left[t^{*}, t\right]}^{2}\right\} \\
& \leq \max \left\{y_{1}^{2}\left(t^{*}\right), 6\left(1+\alpha\left(\left\|y_{1}\right\|_{L^{\infty}[0, t]}+\left\|y_{0}\right\|_{L^{\infty}[0, t]}\right)\right)\left\|y_{0}\right\|_{L^{\infty}\left[t^{*}, t\right]}^{2}+2\left\|u_{0}\right\|_{L^{\infty}\left[t^{*}, t\right]}^{2}\right\} \\
& \leq \max \left\{y_{1}^{2}\left(t^{*}\right), 6\left(1+\alpha\left(\left\|y_{1}\right\|_{L^{\infty}[0, t]}+\left\|y_{0}\right\|_{L^{\infty}[0, t]}\right)\right)\left\|y_{0}\right\|_{L^{\infty}[0, t]}^{2}+2\left\|u_{0}\right\|_{L^{\infty}[0, t]}^{2}\right\}
\end{aligned}
$$

Letting $t \rightarrow \omega$, and by inequality (103):

$$
\begin{gathered}
\left\|y_{1}\right\|_{L^{\infty}\left[t^{*}, \omega\right)}^{2} \leq \max \left\{\left(y_{1}^{0}\right)^{2}, 6\left(1+a^{2}\right)\left\|y_{0}\right\|_{L^{\infty}[0, \infty)}^{2}+2\left\|u_{0}\right\|_{L^{\infty}[0, \infty)}^{2}+4|a|\left(\frac{1}{\alpha} a^{2}+\left\|y_{0}\right\|_{L^{\infty}[0, \infty)}\right)^{2}\right. \\
\left.6\left(1+\alpha\left(\left\|y_{1}\right\|_{L^{\infty}[0, \omega)}+\left\|y_{0}\right\|_{L^{\infty}[0, \infty)}\right)\right)\left\|y_{0}\right\|_{L^{\infty}[0, \infty)}^{2}+2\left\|u_{0}\right\|_{L^{\infty}[0, \infty)}^{2}\right\}
\end{gathered}
$$

Furthermore,

$$
\begin{aligned}
\left\|y_{1}\right\|_{L^{\infty}[0, \omega) \leq}^{2} \leq & \max \left\{\left\|y_{1}\right\|_{L^{\infty}\left[t^{*}, \omega\right)}^{2},\left\|y_{1}\right\|_{L^{\infty}\left[0, t^{*}\right]}^{2}\right\} \\
\leq & \max \left\{\left(y_{1}^{0}\right)^{2}, 6\left(1+a^{2}\right)\left\|y_{0}\right\|_{L^{\infty}[0, \infty)}^{2}+2\left\|u_{0}\right\|_{L^{\infty}[0, \infty)}^{2}+4|a|\left(\frac{1}{\alpha} a^{2}+\left\|y_{0}\right\|_{L^{\infty}[0, \infty)}\right)^{2}\right. \\
& \left.\quad 6\left(1+\alpha\left(\left\|y_{1}\right\|_{L^{\infty}[0, \omega)}+\left\|y_{0}\right\|_{L^{\infty}[0, \infty]}\right)\right)\left\|y_{0}\right\|_{L^{\infty}[0, \infty]}^{2}+2\left\|u_{0}\right\|_{L^{\infty}[0, \infty]}^{2}, \frac{1}{\alpha} a^{2}+\left\|y_{0}\right\|_{L^{\infty}[0, \infty)}\right\}
\end{aligned}
$$

Since the r.h.s of inequality (165) has linear growth in $\left\|y_{1}\right\|_{L^{\infty}[0, \omega)}$ with positive coefficients, it follows that $\left\|y_{1}\right\|_{L^{\infty}[0, \omega)}$ is bounded as a function of $\left|y_{1}^{0}\right|,|a|,\left\|y_{0}\right\|_{L^{\infty}[0, \infty)},\left\|u_{0}\right\|_{L^{\infty}[0, \infty)}$ and by Lemma $6.1, \omega=\infty$.

A bound for $\left\|u_{1}\right\|_{L^{\infty}[0, \infty)}$ follows similarly to (41):

$$
\left\|u_{1}\right\|_{L^{\infty}[0, \infty)} \leq\left\|u_{0}\right\|_{L^{\infty}[0, \infty)}+\sqrt{\alpha}\left(\left\|y_{0}\right\|_{L^{\infty}[0, \infty)}+\left\|y_{1}\right\|_{L^{\infty}[0, \infty)}\right)^{\frac{3}{2}}+\left\|y_{0}\right\|_{L^{\infty}[0, \infty)}+\left\|y_{1}\right\|_{L^{\infty}[0, \infty)} .
$$

Hence it follows that $\left\|u_{1}\right\|_{L^{\infty}[0, \infty)}$ is bounded as a function of $\left|y_{1}^{0}\right|,|a|,\left\|y_{0}\right\|_{L^{\infty}[0, \infty)},\left\|u_{0}\right\|_{L^{\infty}[0, \infty)}$. We have thus established 1 ).

We now establish 2). Let $\left(u_{0}, y_{0}\right)^{T} \in \mathcal{W}$, and let $[0, \omega)$ be the maximal interval of existence. Suppose $a \geq 0$ and consider the function $V_{1}$

$$
V_{1}\left(y_{1}, \hat{k}\right)=\frac{1}{2} y_{1}^{2}+\frac{1}{2 \alpha}\left(a-\hat{k}^{4}\right)^{2} .
$$

Let $t^{\prime}=\inf \left\{t \geq 0: \hat{k}(t) \geq \min \left\{1, a^{\frac{1}{4}}, a\right\}\right\}$ if the infimum exists, and $t^{\prime}=\omega$ otherwise. Define $t^{*} \leq \omega$ as previously. On $\left[0, t^{\prime}\right)$, we have the following inequality:

$$
\begin{aligned}
\dot{V}_{1} & =-y_{1}^{2}+(a-\hat{k}) y_{1}^{2}+u_{0} y_{1}+\hat{k} y_{0} y_{1}+y_{0} y_{1}-\left(a-\hat{k}^{4}\right) y_{2}^{2} \\
& \leq-y_{1}^{2}+\left(u_{0}+(\hat{k}+1) y_{0}+2\left(a-\hat{k}^{4}\right) y_{0}\right) y_{1}-\left(a-\hat{k}^{4}\right) y_{0}^{2} \\
& \leq-y_{1}^{2}+\left(\left\|u_{0}\right\|_{L^{\infty}[0, \infty)}+\left(a^{\frac{1}{4}}+2 a+1\right)\left\|y_{0}\right\|_{L^{\infty}[0, \infty)}\right)\left|y_{1}\right| .
\end{aligned}
$$

Since on $\left[0, t^{\prime}\right), \hat{k}$ is increasing and $\hat{k} \leq a^{\frac{1}{4}}$, it follows that $\frac{1}{2 \alpha}\left(a-\hat{k}^{4}\right)^{2}$ is decreasing. Hence it can be seen that

$$
\frac{1}{2}\left\|y_{1}\right\|_{L^{\infty}\left[0, t^{\prime}\right]}^{2} \leq V_{1}\left(\max \left\{y_{1}^{0},\left\|u_{0}\right\|_{L^{\infty}[0, \infty)}+\left(a^{\frac{1}{4}}+2 a+1\right)\left\|y_{0}\right\|_{L^{\infty}[0, \infty)}\right\}, 0\right) .
$$

Suppose $a>1$ so $t^{*}>t^{\prime}$ and consider $V_{2}: \mathbb{R} \times \mathbb{R} \rightarrow \mathbb{R}_{+}$,

$$
V_{2}\left(y_{1}, \hat{k}\right)=\frac{1}{2} y_{1}^{2}+\frac{a^{3}}{2 \alpha}(a-\hat{k})^{2} .
$$


For $t \in\left[t^{\prime}, t^{*}\right]$, we have the inequality,

$$
\begin{aligned}
\dot{V}_{2} & =-y_{1}^{2}+(a-\hat{k}) y_{1}^{2}+u_{0} y_{1}+y_{0} y_{1}+\hat{k} y_{0} y_{1}-\frac{a^{3}}{\hat{k}^{3}}(a-\hat{k}) y_{2}^{2} \\
& =-y_{1}^{2}+\left(1-\frac{a^{3}}{\hat{k}^{3}}\right)(a-\hat{k}) y_{1}^{2}+u_{0} y_{1}+\left(1+\hat{k}+2 \frac{a^{3}}{\hat{k}^{3}}(a-\hat{k})\right) y_{0} y_{1}-\frac{a^{3}}{\hat{k}^{3}}(a-\hat{k}) y_{0}^{2} \\
& \leq-y_{1}^{2}+\left(\left\|u_{0}\right\|_{L^{\infty}[0, \infty)}+\left(1+a+2 a^{4}\right)\left\|y_{0}\right\|_{L^{\infty}[0, \infty)}\right)\left|y_{1}\right|
\end{aligned}
$$

where we utilize the facts that on $\left[t^{\prime}, t^{*}\right], a \geq \hat{k} \geq \min \left(1, a^{\frac{1}{4}}, a\right)=1$. Since $\frac{a^{3}}{2 \alpha}(a-\hat{k})^{2}$ is decreasing, it can be seen that,

$$
\frac{1}{2}\left\|y_{1}\right\|_{L^{\infty}\left[t^{\prime}, t^{*}\right]}^{2} \leq V_{2}\left(\max \left\{y_{1}\left(t^{\prime}\right),\left\|u_{0}\right\|_{L^{\infty}[0, \infty)}+\left(1+a+2 a^{4}\right)\left\|y_{0}\right\|_{L^{\infty}[0, \infty)}\right\}, \hat{k}\left(t^{\prime}\right)\right)
$$

and

$$
\begin{gathered}
\left\|y_{1}\right\|_{L^{\infty}\left[0, t^{*}\right]}^{2} \leq 2 V_{2}\left(\operatorname { m a x } \left\{\sqrt{2 V_{1}\left(\max \left\{y_{1}^{0},\left\|u_{0}\right\|_{L^{\infty}[0, \infty)}+\left(a^{\frac{1}{4}}+2 a+1\right)\left\|y_{0}\right\|_{L^{\infty}[0, \infty)}\right\}, 0\right)},\right.\right. \\
\left.\left.\left\|u_{0}\right\|_{L^{\infty}[0, \infty)}+\left(1+a+2 a^{4}\right)\left\|y_{0}\right\|_{L^{\infty}[0, \infty)}\right\}, 1\right) .
\end{gathered}
$$

If $0 \leq a \leq 1$ then $t^{*}=t^{\prime}$ then $\left\|y_{1}\right\|_{L^{\infty}\left[0, t^{*}\right]}=\left\|y_{1}\right\|_{L^{\infty}\left[0, t^{\prime}\right]}$. If $a<0$ then $t^{*}=0$. Thus for all $a \in \mathbb{R}$ we have bounded $\left\|y_{1}\right\|_{L^{\infty}\left[0, t^{*}\right]}$ in terms of $y_{1}^{0}, a,\left\|u_{0}\right\|_{L^{\infty}[0, \infty)},\left\|y_{0}\right\|_{L^{\infty}[0, \infty)}$. Noting that for any $a \in \mathbb{R}$, the same argument as used previously to derive inequality (105) gives the following inequalities for $t \in\left[t^{*}, \omega\right):$

$$
\begin{aligned}
\left\|y_{1}\right\|_{L^{\infty}\left[t^{*}, \omega\right)}^{2} & \leq \max \left\{y_{1}^{2}\left(t^{*}\right), 3\left(1+\|\hat{k}\|_{L^{\infty}\left[t^{*}, \omega\right)}^{2}\right)\left\|y_{0}\right\|_{L^{\infty}\left[t^{*}, \infty\right)}^{2}+\left\|u_{0}\right\|_{L^{\infty}\left[t^{*}, \infty\right)}^{2}\right\} \\
& \leq \max \left\{y_{1}^{2}\left(t^{*}\right), 3\left(1+\left|a_{\max }\right|^{2}\right)\left\|y_{0}\right\|_{L^{\infty}\left[t^{*}, \infty\right]}^{2}+\left\|u_{0}\right\|_{L^{\infty}\left[t^{*}, \infty\right]}^{2}\right\}
\end{aligned}
$$

where note that if $a<0$, then $t^{*}=0$, so $y_{1}\left(t^{*}\right)=y_{1}^{0}$. Consequently, by Lemma $6.1, \omega=\infty$.

Hence by inequalities (111), (115) and (116) we have given a bound for $\left\|y_{1}\right\|_{L^{\infty}[0, \infty)}$ in terms of $y_{1}^{0}$, $a, a_{\max },\left\|u_{0}\right\|_{L^{\infty}[0, \infty)},\left\|y_{0}\right\|_{L^{\infty}[0, \infty)}$. Similarly to inequality (108), a bound for $\left\|u_{1}\right\|_{L^{\infty}[0, \infty)}$ is given by:

$$
\left\|u_{1}\right\|_{L^{\infty}[0, \infty)} \leq\left\|u_{0}\right\|_{L^{\infty}[0, \infty)}+\left(1+a_{\max }\right)\left(\left\|y_{0}\right\|_{L^{\infty}[0, \infty)}+\left\|y_{1}\right\|_{L^{\infty}[0, \infty)}\right) .
$$

This completes the proof of 2 .

With respect to the augmented signal space $\widetilde{\mathcal{U}}=E_{n} \times \mathcal{U}$, we define the augmented plant $P$ and controllers $C_{L^{\infty}}, C_{\operatorname{Proj}\left(\theta_{\max }\right)}$ as in Section 4.3, with $n=1, p=\infty$ :

$$
\begin{aligned}
P: \widetilde{\mathcal{U}}_{a} & \rightarrow \mathcal{Y}_{a}, & & P\left(\theta, u_{1}^{*}\right)=P^{*}(\theta, 0)\left(u_{1}^{*}\right) \\
C_{L^{\infty}}: \mathcal{Y}_{a} & \rightarrow \widetilde{\mathcal{U}}_{a}, & & C_{L^{\infty}}\left(y_{2}\right)=\left(0, C_{L^{\infty}}^{*}\left(u_{2}^{*}\right)\right) \\
C_{\operatorname{Proj}\left(a_{\max }\right)}: \mathcal{Y}_{a} & \rightarrow \widetilde{\mathcal{U}}_{a}, & & C_{\operatorname{Proj}\left(a_{\max }\right)}^{*}\left(y_{2}\right)=\left(0, C_{\operatorname{Proj}\left(a_{\max }\right)}\left(u_{2}^{*}\right)\right),
\end{aligned}
$$

where $\theta=(a, 1,1)$ and $C_{L^{\infty}}^{*}, C_{\operatorname{Proj}\left(a_{\max }\right)}^{*}$ are given by (99) and (98) respectively.

Proposition 6.3. Let $\mathcal{U}=\mathcal{Y}=L^{\infty}[0, \infty)$ and let $\Omega_{1}=\mathbb{R} \times\{1\} \times\{1\}, \Omega_{2}=\left(-\infty, a_{\max }\right] \times\{1\} \times\{1\}$. Then both $\left.H_{P, C_{L} \infty}\right|_{\Omega_{1} \times \mathcal{W}}$ and $\left.H_{P, C_{\operatorname{Proj}\left(a_{\max }\right)}}\right|_{\Omega_{2} \times \mathcal{W}}$ are gain function stable.

Proof. Explicit gain function bounds with the above properties follow from Proposition 6.2 by similar calculations to that of Proposition 4.3. 
The following theorem can now be stated:

Theorem 6.4. Let $\mathcal{U}=\mathcal{Y}=L^{\infty}[0, \infty)$, and consider $P^{*}\left((a, 1,1), y_{1}^{0}\right): \mathcal{U}_{a} \rightarrow \mathcal{Y}_{a}$ :

$$
P^{*}\left((a, 1,1), y_{1}^{0}\right)\left(u_{1}\right): \dot{y}_{1}=a y_{1}+u_{1}, y_{1}(0)=y_{1}^{0} \in \mathbb{R}, a \in \mathbb{R} \text {. }
$$

Then:

1. There exists a function $\mu_{1}: \mathbb{R}_{+} \times\left(-\infty, a_{\max }\right] \rightarrow(0, \infty)$ such that if $\theta_{1} \in \mathcal{L}$ satisfies the following inequality:

$$
\vec{\delta}_{L^{\infty}}\left(P^{*}((a, 1,1), 0), P^{*}\left(\theta_{1}, 0\right)\right) \leq \mu_{1}\left(r_{0}, a\right),
$$

for some $a \in \mathbb{R}$ and $r_{0}>0$, then there exists $\lambda>0$ such that $\lambda\left|x_{1}^{0}\right|+\left\|\left(u_{0}, y_{0}\right)^{T}\right\|_{L^{\infty}[0, \infty)} \leq r_{0}$ implies $H_{P^{*}\left(\theta_{1}, x_{1}^{0}\right), C_{\operatorname{Proj}\left(a_{\max }\right)}^{*}}\left(u_{0}, y_{0}\right)^{T}$ is bounded in $L^{\infty}[0, \infty)$.

2. There exists a function $\mu_{2}: \mathbb{R}_{+} \times \mathbb{R} \rightarrow(0, \infty)$ such that if $\theta_{1} \in \mathcal{L}$ satisfies the following inequality:

$$
\vec{\delta}_{L^{\infty}}\left(P^{*}\left((a, 1,1), y_{1}^{0}\right), P^{*}\left(\theta_{1}, 0\right)\right) \leq \mu_{2}\left(r_{0}, a\right)
$$

for some $a \in \mathbb{R}$ and $r_{0}>0$, then there exists $\lambda>0$ such that $\lambda\left|x_{1}^{0}\right|+\left\|\left(u_{0}, y_{0}\right)^{T}\right\|_{L^{\infty}[0, \infty)} \leq r_{0}$ implies $H_{P^{*}\left(\theta_{1}, x_{1}^{0}\right), C_{L}^{*}}\left(u_{0}, y_{0}\right)^{T}$ is bounded in $L^{\infty}[0, \infty)$.

Proof. The proof follows from Proposition 6.3 by a similar construction to that of Theorem 3.1.

The two solutions possess very different characteristics. As with all projection modifications, the projection design considered here requires a-priori knowledge of the range of parameter values for $a$, and whose performance can be expected to scale poorly as $a_{\max }$ becomes a conservative bound for $a$, see eg. [18] for a detailed discussion in a similar context (in the language of Section 7 below, the projection design is conservative, whilst the $L^{\infty}$ controller is universal). On the other hand, the projection design will behave identically to the non-modified $L^{2}$ controller if the disturbances are sufficiently small in $L^{2}$ (ie. if $\hat{k}$ never reaches $a_{\max }$ ), and hence, in this scenario, additionally inherits the positive $L^{2}$ robustness guarantees. Conversely, the $L^{\infty}$ controller requires no a-priori knowledge of the uncertainty level so there is no scaling issue, but $L^{2}$ or asymptotic gain properties of this controller are not immediate and require further analysis - this remains a topic of current research.

\subsection{Applications}

It has been shown in [8] that both the series connections of all pass factors $\left(\frac{M-s}{M+s}\right)$, and first order lags $\left(\frac{M}{s+M}\right)$ to the nominal plant $P^{*}(\theta, 0)$ form arbitrarily small perturbations in the $L^{\infty}$ gap as $M \rightarrow \infty$. It can also be shown that the generalised Rohrs example (equation (21)) leads to an arbitrary small gap perturbation around the nominal plant $P^{*}(\theta, 0)$. Rohrs considered stabilisation with a constant disturbance (p887, figure 10). Robustness in the presence of these unmodelled dyanmics follows from Corollary 3.2.1.

\section{A rationale for adaptive control}

The classical adaptive control problems can be specified via an uncertainty set which we will denote by $\Delta_{\infty} \subset \mathcal{L}$, and which typically contains a non-compact set of nominal plants. The control task is to 
construct a single controller to stabilize all plants within the set. Thus we define a controller $C^{*}$ to be universal (w.r.t. $\Delta_{\infty}$ ) if $C^{*}$ stabilizes $P^{*}(\theta, 0)$ for all $\theta \in \Delta_{\infty}{ }^{4}$

In practice, the requirement of a single controller which stabilises the plant irrespective of the level of uncertainty is overly strong. It is hard to envisage a physical problem in which there is no information about the uncertainty level. We therefore relax the notion of universality as follows (see also [3]). Let $\{\Delta(\beta)\}_{\beta \geq 0}$ be a parameterised collection of nested subsets of $\Delta_{\infty}$ such that $\cup_{\beta \geq 0} \Delta(\beta)=\Delta(\infty)=\Delta_{\infty}$. Here, the parameter $\beta$ represents the uncertainty level, i.e. the 'size' of the uncertainty set. To capture the notion of performance, let $D \subset \mathcal{W}$ be bounded, and define $\mathcal{P}_{D}\left(\left[P^{*}(\Delta(\beta), 0), C^{*}\right]\right)$ to be the worst case cost:

$$
\mathcal{P}_{D}\left(\left[P^{*}(\Delta(\beta), 0), C^{*}\right]\right)=\sup _{\theta \in \Delta(\beta)} \sup _{\left(u_{0}, y_{0}\right)^{T} \in D}\left\|\Pi_{P^{*}(\theta, 0) / / C^{*}}\left(\begin{array}{c}
u_{0} \\
y_{0}
\end{array}\right)\right\|_{\mathcal{W}} .
$$

It is realistic that a (possibly very conservative) upper bound $\beta^{*}$ for $\beta$ is available. It is therefore reasonable to allow the controller to possibly depend on $\beta^{*}$, provided that the performance of the controller does not degrade as $\beta^{*}$ becomes more conservative. Universal controllers automatically do not degrade as $\beta^{*}$ becomes conservative simply because they are independent of $\beta^{*}$ (see [23] and [3] for some related ideas). This provides a practical motivation for the classical problem of constructing universal adaptive controllers even when bound $\beta^{*} \geq \beta$ is known.

Defining $\mathcal{C}$ to be the set of all causal controllers $\mathcal{Y}_{a} \rightarrow \mathcal{U}_{a}$, we make the following definition:

Definition 7.1. Let $D \subset \mathcal{W} . A \mathcal{P}_{D}$ stable control design is a mapping $\Gamma: \mathbb{R}_{+} \rightarrow \mathcal{C}$ such that for all $\beta \geq 0$ and for all $\beta^{*} \geq \beta$,

$$
\mathcal{P}_{D}\left(\left[P^{*}(\Delta(\beta), 0), \Gamma\left(\beta^{*}\right)\right]\right)<\infty .
$$

Definition 7.2. Let $D \subset \mathcal{W}$. A control design $\Gamma: \mathbb{R}_{+} \rightarrow \mathcal{C}$ is said to be:

1. $\mathcal{P}_{D}$ semi-universal if for all $\beta \geq 0$, there exists $\overline{\mathcal{P}}>0$ such that for all $\beta^{*} \geq \beta$,

$$
\mathcal{P}_{D}\left(\left[P^{*}(\Delta(\beta), 0), \Gamma\left(\beta^{*}\right)\right]\right)<\overline{\mathcal{P}},
$$

2. $\mathcal{P}_{D}$ conservative if for all $\beta \geq 0$,

$$
\lim _{\beta^{*} \rightarrow \infty} \mathcal{P}_{D}\left(\left[P^{*}(\Delta(\beta), 0), \Gamma\left(\beta^{*}\right)\right]\right)=\infty .
$$

Note that a universal controller $C^{*}$ has the simple (constant) design mapping $\Gamma(\beta)=C^{*}$, for all $\beta>0$, and hence if it is $\mathcal{P}_{D}$ stable design then it is automatically $\mathcal{P}_{D}$ semi-universal.

To motivate the usefulness of this property, we observe that there is a simple result which shows the superiority of $\mathcal{P}_{D}$ semi-universal control designs over control designs which are $\mathcal{P}_{D}$ conservative when the a-priori known bound on the uncertainty level is sufficiently conservative:

Lemma 7.3. Let $D \subset \mathcal{W}$. Suppose $\Gamma_{1}, \Gamma_{2}$ are $\mathcal{P}_{D}$ stable control designs which are $\mathcal{P}_{D}$ semi-universal and $\mathcal{P}_{D}$ conservative respectively. Then for all $\beta \geq 0$ there exists $\beta^{* *} \geq \beta$ such that, for all $\beta^{*} \geq \beta^{* *}$,

$$
\mathcal{P}_{D}\left(\left[P^{*}(\Delta(\beta), 0), \Gamma_{1}\left(\beta^{*}\right)\right]\right)<\mathcal{P}_{D}\left(\left[P^{*}(\Delta(\beta), 0), \Gamma_{2}\left(\beta^{*}\right)\right]\right) .
$$

Proof. The proof follows directly from the definitions.

\footnotetext{
${ }^{4}$ Note that this definition differs from some other usages of the term universal in adaptive control - often universal is defined to be the concept of stabilizability in the absence of knowledge of the sign of the high frequency gain.
} 
This lemma is given considerable weight since for the types of uncertainty set we have considered to date, namely

$$
\Delta(\beta)=\left\{(a, 1,1) \in E_{1}: a \leq \beta\right\},
$$

any stable control design of the form

$$
\begin{array}{lll}
\Gamma: \mathbb{R}_{+} \rightarrow \mathcal{S} & \text { (Memoryless) } & \text { or } \\
\Gamma: \mathbb{R}_{+} \rightarrow \mathcal{R} & \text { (LTI) }
\end{array}
$$

will be shown to be conservative in sub-sections 7.1, 7.2 below. Here the admissable set of memoryless controllers $\mathcal{S}$ is defined as

$$
\mathcal{S}=\left\{C^{*}: \mathcal{Y}_{a} \rightarrow \mathcal{U}_{a} \mid C^{*}\left(y_{2}\right)=u_{2}, u_{2}(t)=f\left(y_{2}(t)\right), f \in C(\mathbb{R})\right\} .
$$

Observe that both $\mathcal{S}$ and $\mathcal{R}$ are sufficiently powerful controller classes to ensure that such stable control designs exist for the uncertainty description 127. Observe also that the controllers (15), (99) define universal control designs, and thus outperform any memoryless or LTI control designs in the sense of Lemma 7.3.

Lemma 7.3 thus fully justifies the need for the complexity of nonlinear and dynamic controllers even for linear plants: the performance advantage of adaptive controllers, namely the semi-universality property, necessarily requires nonlinear and dynamic controllers. As this performance advantage only requires semi-universality, rather than the stronger universality property we shall henceforth be content to construct semi-universal control designs. Furthermore, these controllers are substantially simpler than the classical solutions [16], [19] and are demonstrated to have non-zero gap robustness margins.

\subsection{Memoryless controllers are conservative}

Relative degree one plants can always be stabilised by memoryless output feedback, where the set of admissable controllers, $\mathcal{C}$, is taken to be $\mathcal{C}=\mathcal{S}$. However this class of control design is $\mathcal{P}_{D}$ conservative:

Proposition 7.4. Let $\mathcal{U}=\mathcal{Y}=L^{2}[0, \infty)$ or $\mathcal{U}=\mathcal{Y}=L^{\infty}[0, \infty)$. Let $r>0$ and $D=B_{r} \subset \mathcal{W}$. Let $\Delta(\beta)$ be given by (127). Suppose $\Gamma: \mathbb{R}_{+} \rightarrow \mathcal{S}$ is a $\mathcal{P}_{D}$ stable design mapping. Then $\Gamma$ is $\mathcal{P}_{D}$ conservative.

Proof. Let $\beta \geq 0, \beta^{*} \geq \beta$ and let $\Gamma\left(\beta^{*}\right)$ denote the controller $u_{2}=f_{\beta^{*}}\left(-y_{2}\right)$, where $f_{\beta^{*}} \in C(\mathbb{R})$. Consider the inputs

$$
\begin{aligned}
& u_{0}= \begin{cases}f_{\beta^{*}}\left(y_{1}\right)+\beta^{*} y_{1} & \text { if } t \leq \tau \\
0 & \text { if } t>\tau,\end{cases} \\
& y_{0}=0,
\end{aligned}
$$

where $\tau \geq 0$ is such that $\left\|u_{0}\right\|_{L^{p}[0, \tau]} \leq r$. Consider $\left[P^{*}\left(\left(\beta^{*}, 1,1\right), 0\right), \Gamma\left(\beta^{*}\right)\right]$ with $u_{0}, y_{0}$ given by $(130)$. Since $\Gamma\left(\beta^{*}\right)$ stabilizes the plant $P^{*}\left(\left(\beta^{*}, 1,1\right), 0\right) \in \Delta\left(\beta^{*}\right)$, and $y_{1}(\tau)=\exp \left(2 \beta^{*} \tau\right)$, it follows that

$$
\beta^{*} y_{1}-f_{\beta^{*}}\left(y_{1}\right) \leq 0, \quad \text { for all } 0 \leq y_{1} \leq \exp \left(2 \beta^{*} \tau\right) .
$$

Now consider $\left[P^{*}((0,1,1), 0), \Gamma\left(\beta^{*}\right)\right]$ with $u_{0}, y_{0}$ given by $(130)$, and let $V: \mathbb{R} \rightarrow \mathbb{R}_{+}$be given by $V\left(y_{1}\right)=\frac{1}{2} y_{1}^{2}$. Then for $t \in[\tau, \tau+1), \dot{y}_{1}=u_{1}=-f_{\beta^{*}}\left(y_{1}\right), y_{1}(\tau)=\exp \left(\beta^{*} \tau\right)$, and

$$
\dot{V} \leq-f_{\beta}^{*}\left(y_{1}\right) y_{1} \leq-\beta^{*} y_{1}^{2},
$$


and so by Cauchy-Schwartz,

$$
\frac{1}{2} \exp \left(2 \beta^{*} \tau\right)=\frac{1}{2} y_{1}^{2}(\tau) \leq \int_{\tau}^{\tau+1} y_{1} \dot{y}_{1} d t \leq\left\|y_{1}\right\|_{L^{2}[\tau, \tau+1]}\left\|u_{1}\right\|_{L^{2}[\tau, \tau+1]} .
$$

Since $\left\|y_{1}\right\|_{L^{2}[\tau, \tau+1]}^{2}=\exp \left(2 \beta^{*} \tau\right) \int_{\tau}^{\tau+1} \exp \left(-2 \beta^{*}(t-\tau)\right) d t=\frac{1}{2 \beta^{*}} \exp \left(2 \beta^{*} \tau\right)$ it follows that

$$
\frac{\sqrt{2 \beta^{*}}}{2} \leq \frac{\sqrt{2 \beta^{*}}}{2} \exp \left(\beta^{*} \tau\right) \leq\left\|u_{1}\right\|_{L^{2}[\tau, \tau+1]} \leq\left\|u_{1}\right\|_{L^{\infty}[\tau, \tau+1]}
$$

hence $\left\|u_{1}\right\|_{L^{2}[0, \infty)},\left\|u_{1}\right\|_{L^{\infty}[0, \infty)} \rightarrow \infty$ as $\beta^{*} \rightarrow \infty$. Since $P^{*}((0,1,1), 0) \in \Delta(\beta)$, this completes the proof for both $p=2$ and $p=\infty$ as required.

\subsection{LTI controllers are conservative}

In this section we show that all proper linear control designs are conservative on our exemplar uncertainty set.

Theorem 7.5. Let $\mathcal{U}=\mathcal{Y}=L^{2}[0, \infty)$ or $\mathcal{U}=\mathcal{Y}=L^{\infty}[0, \infty)$. Let $r>0$ and $D=B_{r} \subset \mathcal{W}$. Let $\Delta(\beta)$ be given by (127). Suppose $\Gamma: \mathbb{R}_{+} \rightarrow \mathcal{R}$ is a $\mathcal{P}_{D}$ stable design mapping. Then $\Gamma$ is $\mathcal{P}_{D}$ conservative.

Proof. Let $p=2$ or $p=\infty$ and $\beta>0$. Since $\Gamma$ is $\mathcal{P}_{D}$ stable, and since $P^{*}((a, 1,1), 0), \Gamma(\beta) \in \mathcal{R}$ it follows that $b_{P^{*}((a, 1,1), 0), \Gamma(\beta)}=\left\|\Pi_{P^{*}((a, 1,1), 0) / / \Gamma(\beta)}\right\|^{-1}>0$ for all $a, \beta \in \mathbb{R}, a<\beta$. Now suppose for a contradiction that $\Gamma$ is not $\mathcal{P}_{D}$ conservative. Let $\epsilon>0$ be fixed. It follows that there exists $b^{*}$ such that $b_{P^{*}((\epsilon, 1,1), 0), \Gamma(\beta)} \geq b^{*}$ for all $\beta>\epsilon$. In particular this implies $\Gamma(\beta)$ stabilizes $\frac{M-s}{(M+s)(s-\epsilon)}$ for sufficiently large $M \geq 0$, since straightforward calculations show

$$
\vec{\delta}_{L^{p}}\left(\frac{1}{s-\epsilon}, \frac{M-s}{(M+s)(s-\epsilon)}\right) \rightarrow 0 \quad \text { as } M \rightarrow \infty .
$$

But since the plant

$$
\frac{2 s^{2}-(\epsilon+\beta) s+(\beta-\epsilon) M}{(s-\beta)(s-\epsilon)(M+s)}=\frac{1}{s-\beta}-\frac{M-s}{M+s} \cdot \frac{1}{s-\epsilon}
$$

is not strongly stabilizable, as there is only one real pole $(\beta)$ between the real zeroes at

$$
s=\frac{\beta+\epsilon+\sqrt{(\beta+\epsilon)^{2}-8(\beta-\epsilon) M}}{4}, \quad s=\infty,
$$

it follows that the plants

$$
\frac{M-s}{M+s} \cdot \frac{1}{s-\epsilon}, \quad \frac{1}{s-\beta}
$$

are not simultaneously stabilizable, [21], thus giving a contradiction.

\section{Robustness of $L^{2}$ and $L^{\infty}$ adaptive controllers under the classical assumptions}

In this section we extend the previous results from the first order nominal plant to linear plants $\widetilde{P}: \mathcal{U}_{a} \rightarrow$ $\mathcal{Y}_{a}$

$$
y_{1}=\widetilde{P}\left(u_{1}\right)=\frac{b_{m} s^{m}+b_{m-1} s^{m-1}+\cdots+b_{0}}{s^{n}+a_{n-1} s^{n-1}+\cdots+a_{0}} u_{1},
$$

which satisfy the classical assumptions of adaptive control namely: 
1. The order of the plant $(n \geq 1)$ is known.

2. The relative degree $(\rho=n-m)$ of the plant is known, and $n \geq \rho \geq 1$.

3. The high frequency gain is positive $\left(\right.$ ie. $\left.b_{m}>0\right) .^{5}$

4. The plant is minimum phase (ie. the polynomial $b_{m} s^{m}+b_{m-1} s^{m-1}+\cdots+b_{0}$ is Hurwitz).

This corresponds to the assumption that $\theta \in \mathcal{M}_{n}^{\rho}$,

$$
\begin{aligned}
& \mathcal{M}_{n}^{\rho}=\left\{\begin{array}{l|l}
\theta=(A, B, C) \in \mathcal{L}_{n} & \begin{array}{l}
C=(1,0, \ldots, 0), C\left(s I_{n}-A\right)^{-1} B \subset \mathbb{C}_{-}, \\
C A^{\xi} B=0,0 \leq \xi \leq \rho-2, C A^{\rho-1} B>0, \\
(A, B) \text { controllable, }(A, C) \text { observable }
\end{array}
\end{array}\right\}, \\
& \mathcal{M}^{\rho}=\cup_{n \geq 1} \mathcal{M}_{n} .
\end{aligned}
$$

\subsection{The relative degree one problem}

We first consider the construction of a universal controller for the relative degree one problem, ie. where $\rho=1$ and $\widetilde{P}(a, b)=P^{*}(\theta, 0)$ satisfies assumptions 1$\left.)-4\right)$, i.e. universal control of the uncertainty set $\mathcal{M}^{1}$

\subsubsection{Stability of the nominal feedback loops}

In the setting of $\mathcal{U}=\mathcal{Y}=L^{2}[0, \infty)$, we consider the controller $C_{L^{2}}^{*}$ defined by (15) and in the setting of $\mathcal{U}=\mathcal{Y}=L^{\infty}[0, \infty)$, we consider the controller $C_{L^{\infty}}^{*}$ defined by $(99)$.

Proposition 8.1. Let $n \geq 1$ and suppose $\left(\theta, x_{1}^{0}\right) \in \mathcal{M}_{n}^{1} \times \mathbb{R}^{n}$.

1. Let $\mathcal{U}=\mathcal{Y}=L^{2}[0, \infty)$. Then the feedback interconnection $\left[P^{*}\left(\theta, x_{1}^{0}\right), C_{L^{2}}^{*}\right]$ is BIBO stable.

2. Let $\mathcal{U}=\mathcal{Y}=L^{\infty}[0, \infty)$. Then the feedback interconnection $\left[P^{*}\left(\theta, x_{1}^{0}\right), C_{L^{\infty}}^{*}\right]$ is BIBO stable.

Proof. Let $n \geq 1$ and suppose $\theta=(A, B, C) \in \mathcal{M}_{n}^{1}$. Let $p=2$ or $p=\infty$. The first part of the proof mirrors the proof of Lemma 2.2.7 in [12], however it is sketched for completeness. Consider the plant under output feedback $u_{2}=-k^{\prime} y_{2}$, with $u_{0}, y_{0}=0$. Since the system is relative degree 1 , the closed loop equations can be expressed in the form:

$$
\frac{d}{d t}\left(\begin{array}{c}
y_{1} \\
z
\end{array}\right)=A_{k^{\prime}} x=\left[\begin{array}{cc}
a_{11}-b_{m} k^{\prime} & a_{12} \\
a_{21} & A_{22}
\end{array}\right]\left(\begin{array}{c}
y_{1} \\
z
\end{array}\right) \quad x(0)=\left[\begin{array}{c}
y_{1}(0) \\
z(0)
\end{array}\right]=0 .
$$

Observe that $x(0)$ can be bounded as a continuous function of $x_{1}^{0}$. As the plant is relative degree one, it can be stabilized by output feedback, ie. there exists $k^{\prime} \geq 0$, s.t. $A_{k^{\prime}}$ is Hurwitz. Then an argument based on Schur's formula:

$$
\operatorname{det}\left(\lambda I-A+B C k^{\prime}\right)=\left|\lambda-a_{11}+b_{m} k^{\prime}\right| \operatorname{det}\left(\lambda-A_{22}-a_{21}\left(\lambda-a_{11}+b_{m} k^{\prime}\right)^{-1} a_{12}\right)
$$

establishes that $\sigma\left(A_{22}\right) \subset \mathbb{C}_{-}$. Let $k=k(\theta) \geq 0$ be such that:

1. $A-k B C$ is Hurwitz,

\footnotetext{
${ }^{5}$ By the appropriate sign changes to the controller, we can also treat the case $b_{m}<0$.
} 
2. $b_{m} k \geq\left|a_{11}\right|+\left|a_{12}\right|+2\left|R a_{21}\right|+\frac{1}{2}\left(\left|a_{12}\right|+2\left|R a_{21}\right|\right)^{2}+\frac{1}{2}-b_{m}$,

where $R$ satisfies the Lyapunov equation:

$$
A_{22}^{T} R+R A_{22}=-I .
$$

Furthermore, this selection of $k$ can be made to be continuously dependent on $\theta$. We now construct two Lyapunov functions. First, since $A-k B C$ is Hurwitz, there exists a positive definite, symmetric matrix $P_{1}$ s.t.

$$
(A-k B C)^{T} P_{1}+P_{1}(A-k B C)=-I .
$$

This defines a Lyapunov function:

$$
V_{1}(x)=x^{T} P_{1} x .
$$

Let $\bar{x}=\left(y_{1}, z^{T}\right)^{T}$, and define the second Lyapunov function:

$$
V_{2}(\bar{x})=\bar{x}^{T} P_{2} \bar{x}=\frac{1}{2} y_{1}^{2}+z^{T} R z .
$$

where $P_{2}$ is given by:

$$
P_{2}=\left[\begin{array}{cc}
\frac{1}{2} & 0 \\
0 & R
\end{array}\right] .
$$

Observe that $P_{1}, P_{2}$ are continuously dependent on $\theta \in M_{1}^{n}$.

Let $\left(u_{0}, y_{0}\right)^{T} \in \mathcal{W}$ and let $[0, \omega)$ be the maximal interval of existence. Let $0 \leq t^{*}<\omega$ be defined:

$$
t^{*}=\inf \{t \geq 0: \hat{k}(t)=k\} .
$$

if the infimum exists, and $t^{*}=\omega$ otherwise.

We now establish two critical inequalities $(148),(150)$. The first key inequality holds on $\left[0, t^{*}\right)$ :

$$
\begin{aligned}
\dot{V}_{1} & =x^{T} P_{1}\left(A x+B u_{1}\right)+\left(A x+B u_{1}\right)^{T} P_{1} x \\
& =x^{T} P_{1}\left(A x+B\left(u_{0}-u_{2}\right)\right)+\left(A x+B\left(u_{0}-u_{2}\right)\right)^{T} P_{1} x \\
= & x^{T} P_{1}\left(A x+B u_{0}+B(\hat{k}+1) y_{2}\right)+\left(A x+B u_{0}+B u_{0}+B(\hat{k}+1) y_{2}\right)^{T} P_{1} x \\
= & x^{T} P_{1}\left(A x+B u_{0}+B(\hat{k}+1)\left(y_{0}-y_{1}\right)\right)+\left(A x+B u_{0}+B u_{0}+B(\hat{k}+1)\left(y_{0}-y_{1}\right)\right)^{T} P_{1} x \\
= & x^{T} P_{1}(A-B C k) x+x^{T}(A-B C k)^{T} P_{1} x+x^{T} P_{1}\left(B u_{0}+B(\hat{k}+1) y_{0}+B(k-\hat{k}-1) y_{1}\right) \\
& \quad+\left(B u_{0}+B(\hat{k}+1) y_{0}+B(k-\hat{k}-1) y_{1}\right)^{T} P_{1} x \\
& -x^{T} x+x^{T} P_{1}\left(B u_{0}+B(\hat{k}+1) y_{0}+B(k-\hat{k}-1) y_{1}\right) \\
& \quad-\left(B u_{0}+B(\hat{k}+1) y_{0}+B(k-\hat{k}-1) y_{1}\right)^{T} P_{1} x
\end{aligned}
$$


The second key inequality holds on $\left[t^{*}, \omega\right)$ :

$$
\begin{aligned}
& \dot{V}_{2}=\left(\left(a_{11}-b_{m}(\hat{k}+1)\right) y_{1}+a_{12} z\right) y_{1}+z^{T} R\left(a_{21} y_{1}+A_{22} z\right)+\left(\left(a_{21} y_{1}+A_{22} z\right)^{T} R z\right. \\
& +\bar{x}^{T} P_{2}\left(\bar{B} u_{0}+\bar{B}(\hat{k}+1) y_{0}\right)+\left(\bar{B} u_{0}+\bar{B}(\hat{k}+1) y_{0}\right)^{T} P_{2} \bar{x} \\
& \leq\left(a_{11}-b_{m}(\hat{k}+1)+\left|a_{12}\right|+2\left|R a_{21}\right|\right) y_{1}^{2}-z^{T} z+\left(a_{12} z\right) y_{1}+z^{T} R\left(a_{21} y_{1}\right)+\left(a_{21} y_{1}\right)^{T} R z \\
& +\bar{x}^{T} P_{2}\left(\bar{B} u_{0}+\bar{B}(\hat{k}+1) y_{0}\right)+\left(\bar{B} u_{0}+\bar{B}(\hat{k}+1) y_{0}\right)^{T} P_{2} \bar{x} \\
& \leq\left(a_{11}-b_{m}(\hat{k}+1)+\left|a_{12}\right|+2\left|R a_{21}\right|\right) y_{1}^{2}-z^{T} z \\
& +\left(\left|a_{12}\right|+2\left|R a_{21}\right|\right)|z|\left|y_{1}\right|+2|\bar{x}||\bar{B}||| P_{2} \|\left(\left|u_{0}\right|+|(\hat{k}+1)|\left|y_{0}\right|\right) \\
& \leq\left(a_{11}-b_{m}(\hat{k}+1)+\left|a_{12}\right|+2\left|R a_{21}\right|+\frac{1}{2}\left(\left|a_{12}\right|+2\left|R a_{21}\right|\right)^{2}\right) y_{1}^{2} \\
& -\frac{1}{2} z^{T} z+2|\bar{x}||\bar{B}||| P_{2} \|\left(\left|u_{0}\right|+|(\hat{k}+1)|\left|y_{0}\right|\right) \\
& \leq-\frac{1}{2} \bar{x}^{T} \bar{x}+2|\bar{x}||\bar{B}||| P_{2} \|\left(\left|u_{0}\right|+|(\hat{k}+1)|\left|y_{0}\right|\right) \\
& \leq-\frac{1}{4}|\bar{x}|^{2}+16|\bar{B}|^{2}\left\|P_{2}\right\|^{2}\left(u_{0}^{2}+3 \hat{k}^{2} y_{0}^{2}+3 y_{0}^{2}\right),
\end{aligned}
$$

where observe that for $t \in[0, \omega)$, the closed loop equations are given by:

$$
\begin{aligned}
\frac{d}{d t}\left[\begin{array}{c}
y_{1} \\
z
\end{array}\right] & =A_{\hat{k}} \bar{x}+\bar{B} u_{1} \\
& =\left[\begin{array}{cc}
a_{11}-b_{m}(\hat{k}(t)+1) & a_{12} \\
a_{21} & A_{22}
\end{array}\right]\left[\begin{array}{c}
y_{1} \\
z
\end{array}\right]+\left[\begin{array}{c}
b_{m} \\
0
\end{array}\right]\left((\hat{k}+1) y_{0}+u_{0}\right), \\
x(0) & =\left[\begin{array}{c}
y_{1}(0) \\
z(0)
\end{array}\right]=0 .
\end{aligned}
$$

Let us consider first consider 1 ), ie. the case where $p=2$. Now we bound $x\left(t^{*}\right)^{T} P_{1} x\left(t^{*}\right)$. Firstly $\left\|y_{1}\right\|_{L^{2}\left[0, t^{*}\right]} \leq\left\|y_{2}\right\|_{L^{2}\left[0, t^{*}\right]}+\left\|y_{0}\right\|_{L^{2}\left[0, t^{*}\right]}=\frac{1}{\sqrt{\alpha}} \hat{k}\left(t^{*}\right)^{2}+\left\|y_{0}\right\|_{L^{2}\left[0, t^{*}\right]} \leq \frac{1}{\sqrt{\alpha}} k^{2}+\left\|y_{0}\right\|_{L^{2}\left[0, t^{*}\right]} \leq \frac{1}{\sqrt{\alpha}} k^{2}+\left\|y_{0}\right\|_{L^{2}[0, \infty)}$.

By applying Young's inequality $\left(a b-\frac{1}{4} b^{2} \leq a^{2}\right)$ three times to (148) we obtain:

$$
\dot{V}_{1} \leq 3|B|^{2}\left\|P_{1}\right\|^{2}\left(\left|u_{0}\right|^{2}+|\hat{k}+1|^{2}\left|y_{0}\right|^{2}+(|k|+|\hat{k}|+1)^{2}\left|y_{1}\right|^{2}\right)
$$

Observing that $\hat{k}$ is non-negative and increasing, and by integrating, we obtain:

$$
\begin{aligned}
V_{1}\left(x\left(t^{*}\right)\right) & -V_{1}(x(0))=\int_{0}^{t^{*}} \dot{V}_{1} d t \\
& \leq 3|B|^{2}\left\|P_{1}\right\|^{2}\left(\left(\hat{k}\left(t^{*}\right)+1\right)^{2}\left\|y_{0}\right\|_{L^{2}\left[0, t^{*}\right]}^{2}+\left\|u_{0}\right\|_{L^{2}\left[0, t^{*}\right]}^{2}+\left(|k|+\left|\hat{k}\left(t^{*}\right)\right|+1\right)^{2}\left\|y_{1}\right\|_{L^{2}\left[0, t^{*}\right]}^{2}\right) \\
& \leq 3|B|^{2}\left\|P_{1}\right\|^{2}\left((k+1)^{2}\left\|y_{0}\right\|_{L^{2}\left[0, t^{*}\right]}^{2}+\left\|u_{0}\right\|_{L^{2}\left[0, t^{*}\right]}^{2}+(2 k+1)^{2}\left\|y_{1}\right\|_{L^{2}\left[0, t^{*}\right]}^{2}\right),
\end{aligned}
$$

which implies:

$$
\begin{aligned}
x\left(t^{*}\right)^{T} P_{1} x\left(t^{*}\right) \leq & V_{1}(x(0))+3|B|^{2}\left\|P_{1}\right\|^{2}\left((k+1)^{2}\left\|y_{0}\right\|_{L^{2}\left[0, t^{*}\right]}^{2}+\left\|u_{0}\right\|_{L^{2}\left[0, t^{*}\right]}^{2}+(2 k+1)^{2}\left\|y_{1}\right\|_{L^{2}\left[0, t^{*}\right]}^{2}\right), \\
\leq & x(0)^{T} P_{1} x(0)+3|B|^{2}\left\|P_{1}\right\|^{2}\left((k+1)^{2}\left\|y_{0}\right\|_{L^{2}[0, \infty]}^{2}+\left\|u_{0}\right\|_{L^{2}[0, \infty]}^{2}\right. \\
& \left.+(2 k+1)^{2}\left(\frac{1}{\sqrt{\alpha}} k^{2}+\left\|y_{0}\right\|_{L^{2}[0, \infty)}\right)^{2}\right) .
\end{aligned}
$$


We now consider the $L^{2}$ estimates on $\left[t^{*}, \omega\right)$. Integrating $(150)$ on $\left[t^{*}, t\right), t<\omega$, we obtain:

$$
\begin{aligned}
V_{2}(\bar{x}(t)) & -V_{2}\left(\bar{x}\left(t^{*}\right)\right)=\int_{t^{*}}^{t} \dot{V}_{2} d t \\
& \leq-\frac{1}{4}\|\bar{x}\|_{L^{2}\left[t^{*}, t\right)}^{2}+48|\bar{B}|^{2}\left\|P_{2}\right\|^{2}\left(\hat{k}^{2}(t)+1\right)\left\|y_{0}\right\|_{L^{2}\left[t^{*}, t\right)}^{2}+16|\bar{B}|^{2}\left\|P_{2}\right\|^{2}\left\|u_{0}\right\|_{L^{2}\left[t^{*}, t\right)}^{2}
\end{aligned}
$$

which implies that for all $t \in\left[t^{*}, \omega\right)$,

$$
\begin{aligned}
\left\|y_{1}\right\|_{L^{2}\left[t^{*}, t\right)}^{2} & \leq\|\bar{x}\|_{L^{2}\left[t^{*}, t\right)}^{2} \\
& \leq 4 \bar{x}\left(t^{*}\right)^{T} P_{2} \bar{x}\left(t^{*}\right)+192|\bar{B}|^{2}\left\|P_{2}\right\|^{2}\left(\hat{k}^{2}(t)+1\right)\left\|y_{0}\right\|_{L^{2}\left[t^{*}, t\right)}^{2}+64|\bar{B}|^{2}\left\|P_{2}\right\|^{2}\left\|u_{0}\right\|_{L^{2}\left[t^{*}, t\right)}^{2} .
\end{aligned}
$$

Now let us estimate $\hat{k}(t)$ for $t \in\left[t^{*}, \omega\right)$. As previously (inequality (37)), we have:

$$
\hat{k}^{2}(t) \leq \sqrt{3 \alpha}\left\|y_{1}\right\|_{L^{2}\left[t^{*}, t\right)}\left(1+\frac{k^{4}+3 \alpha\left\|y_{0}\right\|_{L^{2}\left[t^{*}, t\right)}^{2}}{6 \alpha\left\|y_{1}\right\|_{L^{2}\left[t^{*}, t\right)}^{2}}\right) .
$$

Substituting inequality (158) into inequality (157),

$$
\begin{aligned}
\left\|y_{1}\right\|_{L^{2}\left[t^{*}, t\right) \leq}^{2} \leq & 4 \bar{x}\left(t^{*}\right)^{T} P_{2} \bar{x}\left(t^{*}\right)+64|\bar{B}|^{2}\left\|P_{2}\right\|^{2}\left\|u_{0}\right\|_{L^{2}\left[t^{*}, t\right)}^{2}, \\
& +192|\bar{B}|^{2}\left\|P_{2}\right\|^{2}\left(\sqrt{3 \alpha}\left\|y_{1}\right\|_{L^{2}\left[t^{*}, t\right)}\left(1+\frac{k^{4}+3 \alpha\left\|y_{0}\right\|_{L^{2}\left[t^{*}, t\right)}^{2}}{6 \alpha\left\|y_{1}\right\|_{L^{2}\left[t^{*}, t\right)}^{2}}\right)+1\right)\left\|y_{0}\right\|_{L^{2}\left[t^{*}, t\right)}^{2} .
\end{aligned}
$$

Rearranging and letting $t \rightarrow \omega$ :

$$
\begin{aligned}
\left\|y_{1}\right\|_{L^{2}\left[t^{*}, \omega\right)}^{3} \leq & \left(192 \sqrt{3 \alpha}|\bar{B}|^{2}\left\|P_{2}\right\|^{2}\left\|y_{0}\right\|_{L^{2}\left[t^{*}, \infty\right)}\right)\left\|y_{1}\right\|_{L^{2}\left[t^{*}, \omega\right)}^{2} \\
& +\left(4 \bar{x}\left(t^{*}\right)^{T} P_{2} \bar{x}\left(t^{*}\right)+64|\bar{B}|^{2}\left\|P_{2}\right\|^{2}\left\|u_{0}\right\|_{L^{2}\left[t^{*}, \infty\right)}^{2}+192|\bar{B}|^{2}\left\|P_{2}\right\|^{2}\left\|y_{0}\right\|_{\left[t^{*}, \infty\right)}^{2}\right)\left\|y_{1}\right\|_{L^{2}\left[t^{*}, \omega\right)} \\
& +\frac{96|\bar{B}|^{2}\left\|P_{2}\right\|^{2}}{\sqrt{3 \alpha}}\left(k^{4}+3 \alpha\left\|y_{0}\right\|_{L^{2}\left[t^{*}, \infty\right)}^{2}\right)\left\|y_{0}\right\|_{L^{2}\left[t^{*}, \infty\right)}^{2} \\
\leq & \left(192 \sqrt{3 \alpha}|\bar{B}|^{2}\left\|P_{2}\right\|\left\|y_{0}\right\|_{L^{2}[0, \infty)}\right)\left\|y_{1}\right\|_{L^{2}\left[t^{*}, \omega\right)}^{2} \\
& \quad+\left(4 \bar{x}\left(t^{*}\right)^{T} P_{2} \bar{x}\left(t^{*}\right)+64|\bar{B}|^{2}\left\|P_{2}\right\|^{2}\left\|u_{0}\right\|_{L^{2}[0, \infty)}^{2}+192|\bar{B}|^{2}\left\|P_{2}\right\|^{2}\left\|y_{0}\right\|_{[0, \infty)}^{2}\right)\left\|y_{1}\right\|_{L^{2}\left[t^{*}, \omega\right)} \\
& \quad+\frac{96|\bar{B}|^{2}\left\|P_{2}\right\|^{2}}{\sqrt{3 \alpha}}\left(k^{4}+3 \alpha\left\|y_{0}\right\|_{L^{2}[0, \infty)}^{2}\right)\left\|y_{0}\right\|_{L^{2}[0, \infty)}^{2}
\end{aligned}
$$

Since the right hand side of inequality (161) is quadratic in $\left\|y_{1}\right\|_{L^{2}\left[t^{*}, \omega\right)}$ with positive coefficients, it follows that $\left\|y_{1}\right\|_{L^{2}\left[t^{*}, \omega\right)}$ is bounded as a function of $\bar{x}\left(t^{*}\right)^{T} P_{2} \bar{x}\left(t^{*}\right),|k|, \theta,\left\|y_{0}\right\|_{L^{2}[0, \infty)},\left\|u_{0}\right\|_{L^{2}[0, \infty)}$. Since we have bounded $\left\|y_{1}\right\|_{L^{2}\left[0, t^{*}\right)}$ in terms of $|k|,\left\|y_{0}\right\|_{L^{2}[0, \infty)}$, and $x\left(t^{*}\right)^{T} P_{1} x\left(t^{*}\right)$ (and hence $\left.\bar{x}\left(t^{*}\right)^{T} P_{2} \bar{x}\left(t^{*}\right)\right)$ in terms of $\left|x_{1}^{0}\right|,|k|, \theta,\left\|y_{0}\right\|_{L^{2}[0, \infty)},\left\|u_{0}\right\|_{L^{2}[0, \infty)}$ it follows that we have bounded $\left\|y_{1}\right\|_{L^{2}[0, \omega)}$ in terms of $\left|x_{1}^{0}\right|$, $|k|, \theta,\left\|y_{0}\right\|_{L^{2}[0, \infty)},\left\|u_{0}\right\|_{L^{2}[0, \infty)}$, as required. By Lemma 4.1, it follows that $\omega=\infty$.

The bound (41) also holds for $\left\|u_{1}\right\|_{L^{2}[0, \infty)}$ :

$$
\left\|u_{1}\right\|_{L^{2}[0, \infty)} \leq\left\|u_{0}\right\|_{L^{2}[0, \infty)}+\alpha^{\frac{1}{4}}\left(\left\|y_{0}\right\|_{L^{2}[0, \infty)}+\left\|y_{1}\right\|_{L^{2}[0, \infty)}\right)^{\frac{3}{2}}+\left\|y_{0}\right\|_{L^{2}[0, \infty)}+\left\|y_{1}\right\|_{L^{2}[0, \infty)}
$$

Hence it follows that $\left\|u_{1}\right\|_{L^{2}[0, \infty)}$ is bounded as a function of $\left|x_{1}^{0}\right|,|k|, \theta,\left\|y_{0}\right\|_{L^{2}[0, \infty)}$ and $\left\|u_{0}\right\|_{L^{2}[0, \infty)}$. This completes the proof of 1 ). 
Now let us consider 2), ie. the case $p=\infty$. Then:

$\left\|y_{1}\right\|_{L^{\infty}\left[0, t^{*}\right]} \leq\left\|y_{2}\right\|_{L^{\infty}\left[0, t^{*}\right]}+\left\|y_{0}\right\|_{L^{\infty}\left[0, t^{*}\right]}=\frac{1}{\alpha} \hat{k}\left(t^{*}\right)^{2}+\left\|y_{0}\right\|_{L^{\infty}\left[0, t^{*}\right]} \leq \frac{1}{\alpha} k^{2}+\left\|y_{0}\right\|_{L^{\infty}\left[0, t^{*}\right]} \leq \frac{1}{\alpha} k^{2}+\left\|y_{0}\right\|_{L^{\infty}[0, \infty)}$.

By inequality (148) we can bound $x\left(t^{*}\right)^{T} P_{1} x\left(t^{*}\right)$ :

$$
\begin{aligned}
x\left(t^{*}\right)^{T} P_{1} x\left(t^{*}\right) \leq \max & \left\{x(0)^{T} P_{1} x(0),\right. \\
& \left.\quad \underline{\lambda}\left(P_{1}\right)\left(2|B|\left\|P_{1}\right\|\left(\left\|u_{0}\right\|_{L^{\infty}\left[0, t^{*}\right)}+(k+1)\left\|y_{0}\right\|_{L^{\infty}\left[0, t^{*}\right)}+(2 k+1)\left\|y_{1}\right\|_{L^{\infty}\left[0, t^{*}\right)}\right)\right)^{2}\right\}
\end{aligned}
$$

and by inequality (149), we obtain the bound:

$$
\begin{aligned}
\left\|y_{1}\right\|_{L^{\infty}\left[t^{*}, \omega\right)}^{2} & \leq\left\|\bar{x}^{T} P_{2} \bar{x}\right\|_{L^{\infty}\left[t^{*}, \omega\right)}^{2} \\
& \leq \max \left\{\bar{x}\left(t^{*}\right)^{T} P_{2} \bar{x}\left(t^{*}\right), \underline{4} \underline{\lambda}\left(P_{2}\right)|\bar{B}|^{2}\left\|P_{2}\right\|^{2}\left(\left\|u_{0}\right\|_{L^{\infty}\left[t^{*}, \infty\right)}^{2}+\left(\|\hat{k}\|_{L^{\infty}\left[t^{*}, \omega\right)}^{2}+1\right)\left\|y_{0}\right\|_{L^{\infty}\left[t^{*}, \infty\right)}^{2}\right)\right\} \\
& \leq \max \left\{\bar{x}\left(t^{*}\right)^{T} P_{2} \bar{x}\left(t^{*}\right), 4 \underline{\lambda}\left(P_{2}\right)|\bar{B}|^{2}\left\|P_{2}\right\|^{2}\left(\left\|u_{0}\right\|_{L^{\infty}\left[t^{*}, \infty\right)}^{2}+\right.\right. \\
& \left.\left.\left(\alpha\left(\left\|y_{1}\right\|_{L^{\infty}[0, \omega)}+\left\|y_{0}\right\|_{L^{\infty}[0, \omega)}\right)+1\right)\left\|y_{0}\right\|_{L^{\infty}\left[t^{*}, \infty\right)}^{2}\right)\right\},
\end{aligned}
$$

Hence,

$$
\begin{aligned}
\left\|y_{1}\right\|_{L^{\infty}[0, \omega) \leq}^{2} \leq \max \left\{\left\|y_{1}\right\|_{L^{\infty}\left[0, t^{*}\right]}^{2},\left\|y_{1}\right\|_{L^{\infty}\left[t^{*}, \infty\right]}^{2}\right\} \\
\leq \max \left\{x(0)^{T} P_{1} x(0), \frac{1}{\alpha} k^{2}+\left\|y_{0}\right\|_{L^{\infty}[0, \infty)},\right. \\
\quad \underline{4}\left(P_{2}\right)|\bar{B}|^{2}\left\|P_{2}\right\|^{2}\left(\left\|u_{0}\right\|_{L^{\infty}\left[t^{*}, \infty\right)}^{2}+\left(\alpha\left(\left\|y_{1}\right\|_{L^{\infty}[0, \omega)}+\left\|y_{0}\right\|_{L^{\infty}[0, \omega)}\right)+1\right)\left\|y_{0}\right\|_{L^{\infty}\left[t^{*}, \infty\right)}^{2}\right) \\
\left.\quad \underline{\lambda}\left(P_{1}\right)\left(2|B|\left\|P_{1}\right\|\left(\left\|u_{0}\right\|_{L^{\infty}\left[0, t^{*}\right)}+(k+1)\left\|y_{0}\right\|_{L^{\infty}\left[0, t^{*}\right)}+2 k\left\|y_{1}\right\|_{L^{\infty}\left[0, t^{*}\right)}\right)\right)^{2}\right\} .
\end{aligned}
$$

It now follows from inequalities (162), (163) and (164) that we have bounded $\left\|y_{1}\right\|_{L^{\infty}[0, \infty)}$ in terms of $\left|x_{1}^{0}\right|,|k|,\left\|y_{0}\right\|_{L^{\infty}[0, \infty)},\left\|u_{0}\right\|_{L^{\infty}[0, \infty)}$, as required. By Lemma 6.1, it follows that $\omega=\infty$.

Similarly to (161), a bound for $\left\|u_{1}\right\|_{L^{2}[0, \infty)}$ is then given by:

$$
\left\|u_{1}\right\|_{L^{\infty}[0, \infty)} \leq\left\|u_{0}\right\|_{L^{\infty}[0, \infty)}+\sqrt{\alpha}\left(\left\|y_{0}\right\|_{L^{\infty}[0, \infty)}+\left\|y_{1}\right\|_{L^{\infty}[0, \infty)}\right)^{\frac{3}{2}}+\left\|y_{0}\right\|_{L^{\infty}[0, \infty)}+\left\|y_{1}\right\|_{L^{\infty}[0, \infty)} .
$$

Hence it follows that $\left\|u_{1}\right\|_{L^{\infty}[0, \infty)}$ is also bounded as a function of $\left|x_{1}^{0}\right|,|k|,\left\|y_{0}\right\|_{L^{\infty}[0, \infty)},\left\|u_{0}\right\|_{L^{\infty}[0, \infty)}$ as required. This completes the proof of 2 .

\subsubsection{The closed loop is gf-stable}

With respect to the augmented signal space $\widetilde{\mathcal{U}}=E_{n} \times \mathcal{U}$, we define the augmented plant $P$ and controllers $C_{L^{2}}, C_{L^{\infty}}$, as in Section 4.3 , with $n \geq 1$ and $p=2$ or $p=\infty$ :

$$
\begin{array}{rlrl}
P: \widetilde{\mathcal{U}}_{a} & \rightarrow \mathcal{Y}_{a}, & P\left(\theta, u_{1}^{*}\right)=P^{*}(\theta, 0)\left(u_{1}^{*}\right), \\
C_{L^{2}}: \mathcal{Y}_{a} \rightarrow \widetilde{\mathcal{U}}_{a}, & C_{L^{\infty}}\left(y_{2}\right)=\left(0, C_{L^{2}}^{*}\left(u_{2}^{*}\right)\right), \\
C_{L^{\infty}}: \mathcal{Y}_{a} \rightarrow \widetilde{\mathcal{U}}_{a}, & C_{L^{\infty}}\left(y_{2}\right)=\left(0, C_{L^{\infty}}^{*}\left(u_{2}^{*}\right)\right),
\end{array}
$$

where $\theta \in \mathcal{M}$ and $C_{L^{2}}^{*}, C_{L^{\infty}}^{*}$ are defined by (15) and (99) respectively.

We now come to the key result: 
Proposition 8.2. Let $p=2$ or $p=\infty$ and $n \geq 1$, and let $\mathcal{U}=\mathcal{Y}=L^{p}[0, \infty)$. Consider $P, C_{L^{p}}$ defined by (167), (168), (169). Suppose $\Omega \subset \mathcal{M}_{n}^{1}$. Then the operator $\left.H_{P, C_{L} p}\right|_{\Omega \times \mathcal{W}}$ is gain function stable.

Proof. The proof is analogous to Proposition 4.3, and follows from the fact that in the proof of Proposition 8.1, the parameter $k$ can be chosen to depend continuously on $\theta$.

We now give a gap margin result:

Theorem 8.3. Let $\mathcal{U}=\mathcal{Y}=L^{p}[0, \infty)$, where $p=2$ or $p=\infty$, and let $n \geq 1$. Then there exists a controller $C_{L^{p}}^{*}: \mathcal{Y}_{a} \rightarrow \mathcal{U}_{a}$ and a continuous function $\mu: \mathbb{R}_{+} \times \mathcal{M}_{n}^{1} \rightarrow(0, \infty)$ such that if $\theta_{1} \in \mathcal{L}$ satisfies

$$
\vec{\delta}_{L^{p}}\left(P^{*}(\theta, 0), P^{*}\left(\theta_{1}, 0\right)\right) \leq \mu\left(r_{0}, \theta\right)
$$

for some $\theta \in \mathcal{M}_{n}^{1}$ and $r_{0}>0$ then there exists $\lambda>0$ such that

$$
\lambda\left|x_{1}^{0}\right|+\left\|\left(\begin{array}{c}
u_{0} \\
y_{0}
\end{array}\right)\right\|_{L^{p}} \leq r_{0} \Rightarrow H_{P^{*}\left(\theta_{1}, x_{1}^{0}\right), C_{L^{p}}^{*}}\left(\begin{array}{c}
u_{0} \\
y_{0}
\end{array}\right) \in L^{p}[0, \infty) \times L^{p}[0, \infty) .
$$

Proof. The result follows from Proposition 8.2 and Theorem 5.2, similarly to the proof of Theorem 3.1.

\subsection{The general relative degree problem}

A non-conservative controller for general relative degree problem is constructed from the universal relative degree one controllers (15), (99) respectively as follows. Suppose the plant $P^{*}(\theta, 0)$ has relative degree $\rho>1$. Let $z(s)=b_{\rho-1}^{\prime} s^{\rho-1}+\cdots+b_{0}^{\prime}$ be any Hurwitz polynomial of degree $\rho-1$ with $b_{\rho-1}^{\prime}>0$. Then $z(s) P^{*}(\theta, 0)$ is relative degree one, minimum phase and has positive high frequency gain. We can apply the results of the previous section directly to this modified plant, i.e. to the closed loop $\left[z(s) P^{*}(\theta, 0), C_{L^{p}}^{*}\right]$. Assuming for now that $u_{0}=0, y_{0}=0$ observe that the boundedness of signals of $\left[z(s) P^{*}(\theta, 0), C_{L^{p}}^{*}\right]$ is equivalent to the boundedness of signals of $\left[P^{*}(\theta, 0), z(s) C_{L^{p}}^{*}\right]$. However we cannot implement the controller $z(s) C_{L^{p}}^{*}$ as it is non-proper.

However, by including suitable filtering terms, we can obtain a proper controller:

$$
\left(\frac{M_{1}}{s+M_{1}}\right) \ldots\left(\frac{M_{\rho-1}}{s+M_{\rho-1}}\right) z(s) C_{L^{p}}^{*}=F_{M_{1}, \ldots, M_{\rho-1}}(s) z(s) C_{L^{p}}^{*} .
$$

Then we show that the filter terms are small in the gap, i.e. $\vec{\delta}\left(z(s) P^{*}(\theta, 0), F_{M_{1}, \ldots, M_{\rho-1}}(s) z(s) P^{*}(\theta, 0)\right) \rightarrow$ 0 , for appropriate choices of $M_{i} \rightarrow \infty$. By the obvious block diagram manipulation we can then establish stability of

$$
\left[P^{*}(\theta, 0), \frac{M_{1} \ldots M_{\rho-1}}{\left(s+M_{1}\right) \ldots\left(s+M_{\rho-1}\right)} z(s) C_{L^{p}}^{*}\right] .
$$

When non-zero disturbances $u_{0}, y_{0}$ are present, the block diagram manipulations result in transformed input $M(s) z(s) y_{0}$, so it no longer suffices to only consider $L^{p}$ constraints on the disturbances. Instead we constrain $u_{0}, y_{0}$ by the $W^{p, \rho-1}$ Sobolev norm: ${ }^{6}$

$$
D=\left\{\left(u_{0}, y_{0}\right):\left\|\left(u_{0}, y_{0}\right)\right\|_{W^{p, \rho-1}} \leq \gamma\right\} \subset L^{p},
$$

where $p=2$ or $p=\infty$. This will introduce sufficient regularity on the disturbances $y_{0}$ to ensure that the signal $M(s) z(s) y_{0}$ is bounded independent of $M_{1}, \cdots M_{\rho-1}$.

Before we prove the main result, Theorem 8.5, we establish the preliminary proposition:

\footnotetext{
${ }^{6}$ Recall that $\|\cdot\|_{W^{p, q}}$ is defined: $\|f\|_{W^{p, q}}=\sum_{i=0}^{q}\left\|f^{(i)}\right\|_{L^{p}}$.
} 
Proposition 8.4. Let $\mathcal{U}=\mathcal{Y}=L^{p}[0, \infty)$ where $p=2$ or $p=\infty$. Suppose $G(s)$ is a strictly proper finite dimensional linear system. Then for all $k \geq 1$ and for all $\epsilon>0$, there exist $M_{i}^{*} \geq 1,1 \leq i \leq k$ such that for all $M_{i} \geq M_{i}^{*}$,

$$
\vec{\delta}\left(G(s), \frac{M_{1} \ldots M_{k}}{\left(s+M_{1}\right) \ldots\left(s+M_{k}\right)} G(s)\right)<\epsilon .
$$

Proof. Let $p=2$ or $p=\infty$ and let $\epsilon>0$. We claim that for any strictly proper finite dimensional linear system $H(s)$, the set $\Delta\left(H(s), \epsilon_{*}\right)$ defined by:

$$
\Delta\left(H(s), \epsilon_{*}\right)=\left\{P_{1}: \mathcal{U}_{a} \rightarrow \mathcal{Y}_{a}: P_{1}=\frac{M}{s+M} H(s), \quad M \geq \epsilon_{*}^{-1} \geq 1\right\}
$$

is asymptotically null. Then by applying the claim recursively we can show there exists $M_{1}^{*}, \ldots, M_{k}^{*}$ such that for all $M_{i} \geq M_{i}^{*}, 1 \leq i \leq k$ :

$$
\begin{aligned}
\vec{d}\left(G(s), F_{M_{1}, \ldots, M_{k}}(s) G(s)\right) & \leq \vec{d}\left(G(s), F_{M_{1}}(s) G(s)\right)+\sum_{i=1}^{k-1} \vec{d}\left(F_{M_{1}, \ldots, M_{i}}(s) G(s), F_{M_{1}, \ldots, M_{i+1}}(s) G(s)\right) \\
& <\frac{\log (1+\epsilon)}{k}+\frac{\log (1+\epsilon)}{k}+\cdots+\frac{\log (1+\epsilon)}{k}=\log (1+\epsilon),
\end{aligned}
$$

where $\vec{d}(\cdot, \cdot)$ is defined by $\vec{d}(\cdot, \cdot)=\log (1+\vec{\delta}(\cdot, \cdot))$ (recall that $\vec{d}(\cdot, \cdot)$ satisfies the triangle inequality [8]). It then follows that $\vec{\delta}\left(G(s), F_{M_{1}, \ldots, M_{k}}(s) G(s)\right)<\epsilon$ as required.

To prove the claim, we express $\mathcal{G}_{H(s)}$ in the co-prime factorised manner: $\mathcal{G}_{H(s)}=\left(\begin{array}{c}A(s) \\ B(s)\end{array}\right) L^{p}[0, \infty)$ where $A(s), B(s) \in \mathcal{R} \mathcal{H}_{\infty}$ are co-prime and we can take $A(s)$ to be relative degree zero. Then we can express $\mathcal{G}_{\frac{M}{s+M} H(s)}$ in the form: $\mathcal{G}_{\frac{M}{s+M} H(s)}=\left(\begin{array}{c}A(s) \\ B(s) \frac{M}{s+M}\end{array}\right) L^{p}[0, \infty)$ since $A(s), B(s) \frac{M}{s+M} \in \mathcal{R} \mathcal{H}_{\infty}$ are co-prime $(A(s)$ is relative degree zero, and since $A(s), B(s)$ are co-prime, they have no common zeroes, hence neither do $A(s), B(s) \frac{M}{s+M}$, hence $A(s), B(s) \frac{M}{s+M}$ are co-prime).

As $A(s), B(s)$ are co-prime, there exists a $V(s), U(s) \in \mathcal{R H}_{\infty}$ such that $(V(s), U(s))\left(\begin{array}{c}A(s) \\ B(s)\end{array}\right)=1$. Define the mapping $\Phi: \mathcal{G}_{H(s)} \rightarrow \mathcal{G}_{\frac{M}{s+M} H(s)}$ by the symbol:

$$
\Phi:=\left(\begin{array}{c}
A(s) \\
B(s) \frac{M}{s+M}
\end{array}\right)(V(s), U(s)) .
$$

Since $B(s) \in \mathcal{R} \mathcal{H}_{\infty}$ and is strictly proper it follows that $s B(s) \in \mathcal{R} \mathcal{H}_{\infty}$. Now,

$$
\begin{aligned}
\left\|\left.(I-\Phi)\right|_{\mathcal{G}_{H(s)}}\right\|_{L^{2}} & =\left\|\left(\begin{array}{c}
A(s) \\
B(s)
\end{array}\right)(V(s), U(s))-\left(\begin{array}{c}
A(s) \\
B(s) \frac{M}{s+M}
\end{array}\right)(V(s), U(s))\right\|_{H^{\infty}} \\
& \leq\|s B(s)\|_{H^{\infty}}\left\|\frac{1}{s+M}\right\|_{H^{\infty}}\|(V(s), U(s))\|_{H^{\infty}} \\
& \rightarrow 0 \text { as } \quad M \rightarrow \infty .
\end{aligned}
$$

since $\|s B(s)\|_{H^{\infty}},\|(V(s), U(s))\|_{H^{\infty}}$ are independent of $M$ and since

$$
\left\|\frac{1}{s+M}\right\|_{H^{\infty}} \rightarrow 0 \quad \text { as } \quad M \rightarrow \infty .
$$


This completes the proof if $p=2$.

Since for a proper system, $\|\cdot\|_{1} \leq(2 n+1)\|\cdot\|_{H^{\infty}}$ where $n$ is the number of states in a minimal representation, it follows that:

$$
\begin{aligned}
\left\|\left.(I-\Phi)\right|_{\mathcal{G}_{H(s)}}\right\|_{L^{\infty}} & =\left\|\left(\begin{array}{c}
A(s) \\
B(s)
\end{array}\right)(V(s), U(s))-\left(\begin{array}{c}
A(s) \\
B(s) \frac{M}{s+M}
\end{array}\right)(V(s), U(s))\right\|_{1} \\
& \leq(2 n+1)\|s B(s)\|_{H^{\infty}}\left\|\frac{1}{s+M}\right\|_{H^{\infty}}\|(V(s), U(s))\|_{H^{\infty}} \\
& \rightarrow 0 \text { as } \quad M \rightarrow \infty .
\end{aligned}
$$

hence completing the proof.

The general result is as follows:

Theorem 8.5. Let $\mathcal{U}=\mathcal{Y}=L^{p}[0, \infty)$ where $p=2$ or $p=\infty$ and let $n>\rho \geq 1$. Define

$$
D=\left\{\left(u_{0}, y_{0}\right)^{T} \in \mathcal{W}:\left\|u_{0}\right\|_{L^{p}}^{2}+\left\|y_{0}\right\|_{W^{p, \rho-1}}^{2} \leq r^{2}\right\}
$$

where $r \geq 0$. Let $z(s)$ be a Hurwitz polynomial of degree $\rho-1$. Let $\{\Omega(\beta)\}_{\beta>0}$ be a parameterised set of compact subsets of $\mathcal{M}_{n}^{\rho}$ such that $\cup_{\beta \geq 0} \Omega(\beta)=\mathcal{M}_{n}^{\rho}$. Then there exists a function $\mu: \mathbb{R}_{+} \times \mathcal{M}_{n}^{\rho} \rightarrow(0, \infty)$ such that if

$$
\Delta(\beta)=\left\{\theta_{1} \in \mathcal{L} \mid \exists \theta \in \Omega(\beta), \text { s.t. } \vec{\delta}_{L^{p}}\left(z(s) P^{*}(\theta, 0), z(s) P^{*}\left(\theta_{1}, 0\right)\right) \leq \mu(r, \theta)\right\},
$$

and $\mathcal{P}_{D}$ denotes the worst case cost:

$$
\mathcal{P}_{D}\left(\left[P^{*}(\Delta(\beta), 0), C_{L^{p}}^{*}\right]\right)=\sup _{\theta \in \Delta(\beta)} \sup _{\left(u_{0}, y_{0}\right)^{T} \in D}\left\|\Pi_{P^{*}(\theta, 0) / / C_{L^{p}}^{*}}\left(\begin{array}{c}
u_{0} \\
y_{0}
\end{array}\right)\right\|_{L^{p}},
$$

then there exists a $\mathcal{P}_{D}$ semi-universal control design.

Proof. Let $p=2$ or $p=\infty, \beta \geq 0$, and suppose $\left(u_{0}, y_{0}\right)^{T} \in D$. Consider the following control design $\Gamma: \mathbb{R}_{+} \rightarrow \mathcal{C}:$

$$
\Gamma(\beta)=F_{M_{1}^{\beta}, \ldots, M_{\rho-1}^{\beta}}(s) z(s) C_{L^{p}}^{*}:=M^{\beta}(s) z(s) C_{L^{p}}^{*} .
$$

where $C_{L^{p}}^{*}$ is defined by equations (15), (99) and $M_{1}^{\beta}, \ldots M_{\rho-1}^{\beta}$ will be determined. Let $\theta^{\dagger} \in \mathcal{M}_{n}^{1}$ define a state space realisation of $z(s) P^{*}(\theta, 0)$, so $P^{*}\left(\theta^{\dagger}, 0\right)=z(s) P^{*}(\theta, 0)$ and define the augmented plant and controller $P, C$ by:

$$
\begin{aligned}
P\left(\theta^{\dagger}, u_{1}^{*}\right) & =P^{*}\left(\theta^{\dagger}, 0\right)\left(u_{1}^{*}\right), \\
C\left(y_{2}\right) & =\left(0, C_{L^{p}}^{*}\left(y_{2}\right)\right) .
\end{aligned}
$$

We know $[P, C]$ is gf. stable by Proposition 8.2, and by Theorem 5.2, there exists a function $\mu^{\prime}$ s.t. if

$$
\vec{\delta}_{L^{p}}\left(z(s) P^{*}(\theta, 0), z(s) M^{\beta}(s) P^{*}\left(\theta_{1}, 0\right)\right) \leq \mu^{\prime}\left(\left\|\left(u_{0}, y_{0}\right)^{T}\right\|_{L^{p}}, \theta^{\dagger}\right)
$$

then $H_{z(s) M^{\beta}(s) P^{*}\left(\theta_{1}, 0\right), C_{L p}^{*}}\left(u_{0}, y_{0}\right) \in L^{p}[0, \infty) \times L^{p}[0, \infty)$. In fact from the proof of theorems 5.1 and 5.2 we also know that if inequality (186) holds, then there exists $\varepsilon \in K_{\infty}$ and a surjective map $\Psi: \mathcal{D} \rightarrow \mathcal{D}_{1}$ where $\mathcal{D}, \mathcal{D}_{1}$ are defined as in (74), (75) (with $\theta^{\dagger}$ replacing $\theta$ ) such that

$$
g\left[\Pi_{P_{1} / / C}\right](r) \leq g[\Phi] \circ g\left[\Pi_{P / / C}\right] \circ\left(1+\varepsilon^{-1}\right)(r)
$$


where $P_{1}$ denotes the operator $P_{1}:\left(E_{n} \times L^{p}[0, \infty)\right)_{a} \rightarrow L^{p}[0, \infty)_{a}$ :

$$
P_{1}\left(\theta^{\dagger}, u_{1}^{*}\right)=z(s) M^{\beta}(s) P^{*}\left(\theta_{1}, 0\right)\left(u_{1}^{*}\right) .
$$

By (83) we also know

$$
g[\Psi](r) \leq(1+g[I-\Psi])(r) \leq\left(1+2 \vec{\delta}_{L^{p}}\left(z(s) P^{*}(\theta, 0), z(s) M(s) P^{*}\left(\theta_{1}, 0\right)\right)\right) r .
$$

From the triangle inequality we have:

$\vec{d}\left(z(s) P^{*}(\theta, 0), M(s) z(s) P^{*}\left(\theta_{1}, 0\right)\right) \leq \vec{d}\left(z(s) P^{*}(\theta, 0), z(s) P^{*}\left(\theta_{1}, 0\right)\right)+\vec{d}\left(z(s) P^{*}\left(\theta_{1}, 0\right), M(s) z(s) P^{*}\left(\theta_{1}, 0\right)\right)$,

From Proposition 8.4 and since $\Omega(\beta)$ is compact, by choosing $\mu(r, \theta)=\left(1+\mu^{\prime}\left(r, \theta^{\dagger}\right)\right)^{\frac{1}{2}}-1$, we can find $M_{1}^{\beta}, \ldots M_{\rho-1}^{\beta} \geq 1$ such that for all $\theta_{1} \in \Omega(\beta)$ and all $M_{i} \geq M_{i}^{\beta}, 1 \leq i \leq \rho-1$,

$$
\vec{d}\left(z(s) P^{*}(\theta, 0), F_{M_{1}, \ldots, M_{\rho-1}} z(s) P^{*}\left(\theta_{1}, 0\right)\right) \leq \log \left(1+\mu^{\prime}\left(\left\|\left(u_{0}, y_{0}\right)^{T}\right\|_{L^{p}}\right)\right) .
$$

and hence such that inequality (186) is satisfied. Without loss of generality assume that $M_{i}^{\beta^{*}} \geq M_{i}^{\beta}$ for all $\beta^{*} \geq \beta, 1 \leq i \leq \rho-1$. Let $M(s)=F_{M_{1}, \ldots, M_{\rho-1}}$ where $M_{i} \geq M_{i}^{\beta}$ and define

$$
Q(s)=\left(\begin{array}{cc}
I & 0 \\
0 & M^{-1}(s) z^{-1}(s)
\end{array}\right) .
$$

By block diagram manipulations, we know:

$$
\Pi_{P^{*}\left(\theta_{1}, 0\right) / / z(s) M(s) C_{L^{p}}^{*}}\left(u_{0}, y_{0}\right)^{T}=Q(s) \Pi_{z(s) M(s) P^{*}\left(\theta_{1}, 0\right) / / C_{L^{p}}^{*}}\left(u_{0}, M(s) z(s) y_{0}\right)^{T},
$$

hence

$$
\left(\theta^{\dagger}, \Pi_{P^{*}\left(\theta_{1}, 0\right) / / z(s) M(s) C_{L^{p}}^{*}}\left(u_{0}, y_{0}\right)^{T}\right)^{T}=\operatorname{diag}(I, Q(s)) \Pi_{P_{1} / / C}\left(\theta^{\dagger}, u_{0}, M(s) z(s) y_{0}\right)^{T},
$$

so,

$$
\begin{aligned}
\left.\mathcal{P}_{D}\left[P^{*}(\Delta(\beta), 0), C_{L^{p}}^{*}\right]\right) & =\sup _{\theta_{1} \in \Delta(\beta)} \sup _{\left(u_{0}, y_{0}\right) \in D}\left\|\Pi_{P^{*}\left(\theta_{1}, 0\right) / / z(s) M(s) C_{L^{p}}^{*}}\left(u_{0}, y_{0}\right)\right\|_{L^{P}}, \\
& \leq \sup _{\theta_{1} \in \Delta(\beta)} \sup _{\left(u_{0}, y_{0}\right) \in D}\left(1+\|Q(s)\|_{H^{\infty}}\right)\left(g\left[\Pi_{P_{1} / / C}\right]\left(\left\|\left(\theta^{\dagger}, u_{0}, M(s) z(s) y_{0}\right)^{T}\right\|\right)\right) .
\end{aligned}
$$

By inequalities (187), (189), we see that $\Pi_{P_{1} / / C}$ is gf. stable, and that the proof will be complete by showing that $\|Q(s)\|_{H^{\infty}},\left\|u_{0}, M(s) z(s) y_{0}\right\|_{L^{P}}$ are bounded independently of $M_{1}, \ldots M_{\rho-1}$ and uniformly over $u_{0}, y_{0} \in D$.

We first show that if $G(s) \in H^{\infty}$ is strictly proper than $\left\|\frac{s+M}{M} G(s)\right\|_{H^{\infty}} \rightarrow\|G(s)\|_{H^{\infty}}$ as $M \rightarrow \infty$ :

$$
\begin{aligned}
\left\|\frac{s+M}{M} G(s)\right\|_{H^{\infty}} & \leq\left\|\left(\frac{s+M}{M}-1\right) G(s)\right\|_{H^{\infty}}+\|G(s)\|_{H^{\infty}} \\
& \leq \frac{1}{M}\|s G(s)\|_{H^{\infty}}+\|G(s)\|_{H^{\infty}} \\
& \rightarrow\|G(s)\|_{H^{\infty}} \quad \text { as } M \rightarrow \infty .
\end{aligned}
$$

Applying the above result recursively to $G(s)=z(s)^{-1}$, we obtain that $\|Q(s)\|_{H^{\infty}}$ is bounded independently of $M_{1}, \ldots M_{\rho-1}$. 
Observe that for $p=2$ or $p=\infty$, we have $\|M(s)\|_{L^{p}} \leq 2 \rho-1$ for any $M_{1}, \ldots M_{\rho-1} \geq 0$. Hence for $\left(u_{0}, y_{0}\right)^{T} \in D$,

$$
\left\|u_{0}, M(s) z(s) y_{0}\right\|_{L^{P}} \leq\left\|u_{0}\right\|+\|M(s)\|\left\|z(s) y_{0}\right\|_{L^{P}} \leq\left(1+(2 \rho-1) \sum_{i=0}^{\rho-1} b_{i}^{\prime}\right) r
$$

where we have written $z(s)=b_{\rho-1}^{\prime} s^{\rho-1}+\cdots+b_{0}^{\prime}$. Hence $\left\|u_{0}, M(s) z(s) y_{0}\right\|_{L^{p}}$ is also bounded independently of $M_{1}, \ldots M_{\rho-1}$. Thus $\Gamma$ is a $\mathcal{P}_{D}$ adaptive control design.

Some comments are in order. Firstly note that the gap margin is about the nominal relative degree one plant $z(s) P^{*}(\theta, 0)$, not the original plant $P^{*}(\theta, 0)$. Secondly observe that the controller obtained is not universal: the filter coefficients $M_{i}, 1 \leq i \leq \rho$ must be taken to be sufficiently large: they depend on the nominal plant set $\Omega(\beta)$ and the location of the zeroes of $z(s)$. However the controller is $\mathcal{P}_{D}$ semi-universal in the sense of the quadratic cost considered, and hence outperforms $\mathcal{P}_{D}$ conservative designs by Lemma 7.3.

Practical difficulties in the implementation of this controller may arise from the required difference in zero/pole locations of the filter $z(s) M(s)$. Since the poles may be required to be much further to the left of the zeroes, the filter $z(s) M(s)$ may be too sensitive to disturbances for some applications. This sensitivity is captured by the Sobolev norm bound on the disturbances, ie. the constraint that the derivatives of $u_{0}, y_{0}$ are also bounded in $L^{2}$ or $L^{\infty}$. Hence one trade-off within this design is between robustness to unmodelled dynamics and sensitivity to (derivatives of) $u_{0}, y_{0}$. Note however, that for some plants, the above strategy may be perfectly feasible, after all, practical analogue implementation of PID controllers involves approximation of the derivative term $k_{d} s$ by $\frac{M k_{d} s}{s+M}$ with 'large' $M$, justified by a similar reasoning.

\section{Conclusions}

In this paper we have approached the classical problem of robustness of adaptive controllers to unmodelled dynamics within the framework of the nonlinear gap metric. The main idea of the approach is fourfold:

- To augment the input signal space of parametrically uncertain linear plants with a channel representing the uncertain parameters.

- To synthesise the controller to ensure the existence of a certain closed loop gain function, and hence to give rise to a nonlinear gap margin.

- To relate the nonlinear gap margin to the (linear) gap margins on the individual plants, and thus give sufficient conditions for robustness in the presence of bounded disturbances.

- To give sufficient conditions for robustness in the presence of non-zero initial conditions and disturbances by appropriate input and output injection.

Under the classical assumptions of a minimum phase plant with positive high frequency gain, results were given for three classes of plant of increasing complexity, namely, the first order case; the relative degree one case; and the general relative degree case. The final result uses the gap techniques in an intrinsic manner within the controller synthesis.

Whilst the results have been presented in a qualitative manner, the proofs are fully constructive, and in principle can be used to explicitly compute the gap margins. We have refrained from exhibiting the 
margins directly as no attempt has been made to optimise any estimates or to consider alternative control structures. The main contribution of the paper is seen to be the demonstration that adaptive controllers with non-zero gap margins can be constructed. A major open area for future research is therefore to investigate optimising tight bounds for such adaptive problems, under suitably formulated criteria for optimisation (eg. minimizing a weighted functional of the gain function of $H_{P, C}$ ). In particular, the benefits of using increased structural information on the parametric uncertainty of the plant can now be rigorously investigated with respect to the robustness margin. Observe that previously there was no way to systematically quantify the advantages of the use of such information, which perhaps partly explains why theoretical investigations into adaptive control have usually focused on very general parametric uncertainties.

We have presented results in both $L^{2}$ and $L^{\infty}$ settings. Within the $L^{2}$ setting we have analysed the behaviour of controllers closely related to the classical designs; however the robustness margins are only valid for $L^{2}$ disturbance signals, for it is well known that these designs are non-robust in the presence of persistent disturbances. The $L^{2}$ analysis is of interest for two reasons. Firstly it highlights that the lack of robustness of designs is necessarily a combination of unmodelled dynamics and inappropriate disturbances. Appropriate classes of unmodelled dynamics can be tolerated if the disturbances are suitable. For example, the $L^{2}$ theory is entirely appropriate for the consideration of robustness to initial conditions only, and gives stronger results than the $L^{\infty}$ theory. Secondly the neighbourhood of tolerable perturbations in the $L^{2}$ setting are described by the $H^{2}$ gap metric, familiar from linear robust control theory. On the other hand, within the $L^{\infty}$ setting we have synthesised robust adaptive controllers which are robust to both a more practical class of persistent disturbances and to initial conditions. The first design considered is the standard projection modification to the $L^{2}$ design; the second design is not a 'classical' design. Taken as a whole we would like to emphasise that this illustrates that the theory is not tied to a single function space and that the appropriate choice of signal space is inherent in the modelling of the control specification: we expect that many meaningful generalisations are possible with other signal norms. In particular, generalisations to tracking and to time-varying systems in suitable signal space settings remain the subject of current work.

In the case of the relative degree $\rho>1$ result, a fundamental open avenue of research is to investigate alternative controller constructions to capture the inherent trade-off necessary between sensitivity, uncertainty and relative degree. Whilst incremental improvement to the sensitivity of the closed loop to disturbances may be made by alternative design of the filter $z(s) M(s)$, control designs of a different structure may even yield universal designs for $L^{2}$ or $L^{\infty}$ disturbances.

Generalisations to classes of nonlinear plants are also expected to be feasible within the framework described in this paper, as it is essentially the linear growth of the right hand side of the plant equations which plays the key role in the construction of the nonlinear gain function, rather than the linearity of the plant per-se. It is thus anticipated that by an appropriate consideration of the nonlinear growth of a plant the results should readily generalise to some classes of nonlinear plants.

Acknowledgements. The author would like to sincerely thank M.C. Smith for inspiring this work, and for a number of constructive comments on earlier drafts of this paper. The author also wishes to thank E.P. Ryan and A. Ilchmann for many useful discussions, in particular to E.P. Ryan for drawing his attention to a shortcoming in an earlier version of Theorem 5.1. A valuable discussion with G. Vinnicombe concerning Section 7 is also acknowledged. 


\section{References}

[1] J. Doyle, T.T. Georgiou, and M.C. Smith. On the parallel projection operators of a nonlinear feedback system. Systems and Control Letters, 20:79-85, 1993.

[2] A.K. El-Sakkary. The gap metric: Robustness of stabilization of feedback systems. IEEE Trans. on Automatic Control, 30(3):240-247, 1985.

[3] M. French. An analytical comparison between the weighted LQ performance of a robust and an adaptive backstepping design. IEEE Trans. on Automatic Control, 47(4):670-675, 2002.

[4] M. French. Smooth adaptive controllers have discontinuous closed loop operators. In 16th Int. Symp. on Mathematical Theory of Networks and Systems, 2004. CD-Rom proceedings.

[5] M. French, Cs. Szepesvári, and E. Rogers. Uncertainty, performance and model dependency in approximate adaptive nonlinear control. IEEE Trans. on Automatic Control, 45(2):353-58, 2000.

[6] T.T. Georgiou. Differential stability and robust control of nonlinear systems. Mathematics of Control, Signals and Systems, 4:289-306, 1993.

[7] T.T. Georgiou and M.C. Smith. Biased norms and robustness analysis for nonlinear feedback systems. In Proc. of the 36th IEEE Conf. on Decision and Control, pages 642-643, 1997.

[8] T.T. Georgiou and M.C. Smith. Robustness analysis of nonlinear feedback systems. IEEE Trans. on Automatic Control, 42(9):1200-1229, 1997.

[9] T.T. Georgiou and M.C. Smith. Remarks on "Robustness analysis of nonlinear feedback systems". IEEE Trans. on Automatic Control, 46(1):171-172, 2001.

[10] K. Glover and D. McFarlane. Robust stabilization of normalized coprime factor plant descriptions with $H_{\infty}$-bounded uncertainty. IEEE Trans. on Automatic Control, 34:811-830, 1989.

[11] F. Ikhouane and M. Krstić. Robustness of the tuning functions adaptive backstepping design for linear systems. IEEE Trans. on Automatic Control, 43(3):431-437, 1998.

[12] A. Ilchmann. Non-Identifier-Based High-Gain Adaptive Control, volume 189 of Lecture Notes in Control and Information Sciences. Springer Verlag, Berlin, Heidelberg, 1993.

[13] P.A. Ioannou and J. Sun. Robust Adaptive Control. Prentice Hall, New York, 1996.

[14] Z.P. Jiang, A. Teel, and L. Praly. A small gain theorem for ISS systems and applications. Mathematics of Control, Signals and Systems, 7:95-120, 1994.

[15] Z.H. Li and M. Krstić. Optimal design of adaptive tracking controllers for non-linear systems. Automatica, 33(8):1459-1473, 1997.

[16] K.S. Narendra and A.M. Annaswamy. Stable adaptive control. Information and System Sciences Series. Prentice Hall, 1990.

[17] C.E. Rohrs, L. Valavani, M. Athans, and G. Stein. Robustness of continuous-time adaptive control algorithms in the prescence of unmodelled dynamics. IEEE Trans. on Automatic Control, 30(9):881-889, 1985. 
[18] A. Sanei and M. French. Towards a performance theory of robust adaptive control. International Journal of Adaptive Control and Signal Processing, 18(4):403-421, 2004.

[19] S. Sastry and M. Bodson. Adaptive Control - Stability, Convergence and Robustness. Prentice Hall, Englewood Cliffs, New Jersey, 1st edition, 1989.

[20] R. Sepulchre, M. Janković, and P.V. Kokotović, editors. Constructive Nonlinear Control. Communications and Control Engineering. Springer Verlag, 1997.

[21] M. Vidyasagar. Control System Synthesis. MIT Press, Cambridge, 1985.

[22] G. Vinnicombe. Frequency domain uncertainty and the graph topology. IEEE Trans. on Automatic Control, 38:1371-1383, 1993.

[23] G. Zames. volume 222 of Lecture notes in Control and Information Sciences, chapter Towards a general complexity-based theory of identification and adaptive control, pages 208-223. Springer Verlag, 1997.

[24] G. Zames and A. K. El-Sakkary. Unstable systems and feedback: The gap metric. In Proc. of the Allerton Conf., pages 380-385, 1980.

[25] E. Zeidler. Nonlinear Functional Analysis and its Applications I: Fixed Point Theorems. Springer, Berlin, 1986.

[26] K. Zhou, J.C. Doyle, and K. Glover. Robust and Optimal Control. Prentice Hall, New Jersey, 1st edition, 1996. 